\title{
A Decision-Making Framework Using q-Rung Orthopair Probabilistic Hesitant Fuzzy Rough Aggregation Information for the Drug Selection to Treat COVID-19
}

\author{
Attaullah, ${ }^{1}$ Shahzaib Ashraf, ${ }^{2}$ Noor Rehman, ${ }^{2}$ Hussain AlSalman $\mathbb{D}^{3},{ }^{3}$ \\ and Abdu H. Gumaei $\left.{ }^{4}\right)^{4}$ \\ ${ }^{1}$ Department of Mathematics, Abdul Wali Khan University, Mardan 23200, Pakistan \\ ${ }^{2}$ Department of Mathematics and Statistics, Bacha Khan University, Charsadda 24420, Pakistan \\ ${ }^{3}$ Department of Computer Science, College of Computer and Information Sciences, King Saud University Riyadh, \\ Riyadh 11543, Saudi Arabia \\ ${ }^{4}$ Computer Science Department, Faculty of Applied Sciences, Taiz University, Taiz 6803, Yemen
}

Correspondence should be addressed to Abdu H. Gumaei; abdugumaei@gmail.com

Received 30 July 2021; Revised 21 September 2021; Accepted 26 October 2021; Published 4 February 2022

Academic Editor: Borna Abramović

Copyright (c) 2022 Attaullah et al. This is an open access article distributed under the Creative Commons Attribution License, which permits unrestricted use, distribution, and reproduction in any medium, provided the original work is properly cited.

\begin{abstract}
In our current era, a new rapidly spreading pandemic disease called coronavirus disease (COVID-19), caused by a virus identified as a novel coronavirus (SARS-CoV-2), is becoming a crucial threat for the whole world. Currently, the number of patients infected by the virus is expanding exponentially, but there is no commercially available COVID-19 medication for this pandemic. However, numerous antiviral drugs are utilized for the treatment of the COVID-19 disease. Identification of the appropriate antivirus medicine to treat the infection of COVID-19 is still a complicated and uncertain decision. This study's key objective is to develop a novel approach called $q$-rung orthopair probabilistic hesitant fuzzy rough set ( $q$-ROPHFRS), which incorporates the $q$ rung orthopair fuzzy set, probabilistic hesitant fuzzy set, and rough set structures. New $q$-ROPHFR aggregation operators have been established: the $q$-ROPHFR Einstein weighted averaging (q-ROPHFREWA) operator and the $q$-ROPHFR Einstein weighted geometric (q-ROPHFREWG) operator. In this study, we explored some basic features of the developed operators. Afterward, to demonstrate the viability and feasibility of the established decision-making approach in real-world applications, a case study related to selecting drugs for COVID-19 pandemic is addressed. Furthermore, a comprehensive comparison with the $q$-rung orthopair probabilistic hesitant fuzzy rough TOPSIS technique is also presented to illustrate the benefits of the new framework. The obtained results confirm the reliability and effectiveness of the proposed approach for finding uncertainty in realworld decision-making.
\end{abstract}

\section{Introduction}

Wuhan, China, was faced with a dangerous challenge in December 2019, which distorted the health of humans and created global instability. The pneumonia cases were caused by a new virus known as coronavirus 2019 (COVID-19). COVID-19 was unknown; therefore, the government of China controlled Wuhan's traffic to prevent the spread of the infection [1]. Russia, the United States, Brazil, India, and France are the most infected countries in terms of the number of confirmed COVID-19 cases. The World Health
Organization (WHO) recognized COVID-19 as a pandemic by March 2020. Several governments and organizations have been closed down and have implemented strict social distancing processes to prevent virus proliferation. According to a WHO report released on June 13, 2021, more than $176,396,104$ cases of COVID-19 have been reported around the world, resulting in more than 3,810,989 deaths, and a total of 160,398,032 people have been recovered [2]. The virus that causes COVID-19 is primarily spread via the droplets created when someone infected with COVID-19 sneezes, coughs, or exhales. Coronavirus is more harmful to 
those who have a low immune system, are elderly, have diabetes, or have medical problems, especially for those involving the lungs problem [3-6]. Virus propagation can be influenced by various factors, including population density, medical care facilities, climate, and others [7]. Coronaviruses are a vast family of viruses that can cause various diseases in both animals and humans. They mainly cause respiratory tract infections in humans, varying from an ordinary cold towards more severe illness disorders such as Middle East respiratory syndrome (MERS) and severe acute respiratory syndrome (SARS) [8, 9]. Phylogenetic and sequencing analyses have shown that COVID-19 is closely related to a collection of human and bat SARS-like coronaviruses $[4,10,11]$. COVID-19 is believed to have evolved from bats to a greater level of life chains [12-14]. The statistic is shown in Figure 1. Doctors, experts, or medical sections should implement an ideal plan, tests, or techniques for the COVID-19 treatment process to avoid further crisis expansion. The department in the process of establishing strategy must make quick and effective decisions. While making decisions in this situation, individuals are often bound logically instead of entirely reasonable. As a result, it is essential to identify appropriate multicriteria decisionmaking (MCDM) models that recognize human activities to provide individuals with practical ways of responding to emergencies. Dealing with uncertainty and unpredictable information in realistic circumstances has always been challenging. Several tools have been developed to address the complexities and conflicts encountered in real-life activities. Zadeh [15] explored a solution to such problems by establishing the foundations of fuzzy set (FS) theory, in which each element is assigned a membership degree ranging between 0 and 1 . Atanassov [16] extended the idea of FS into intuitionistic FS (IFS) by introducing nonmembership $\left(\psi_{F}(x)\right)$ to the membership $\left(\beta_{F}(x)\right)$ of the FS, with the restriction that $\beta_{\mathrm{F}}(x)+\psi_{\mathrm{F}}(x) \leq 1$.

Yager [17] introduced the Pythagorean FS (PFS) theory, which relaxes the previously mentioned IFS condition to $\left(\beta_{\mathrm{F}}(x)\right)^{2}+\left(\psi_{\mathrm{F}}(x)\right)^{2} \leq 1$. PF expressions are undoubtedly raising the interest of many researchers, especially in terms of their applications to DM. For example, Huang et al. [18] described a PF MULTIMOORA approach that utilizes a novel distance measure and a score function. They used this approach to evaluate disk productions and energy projects. Zhang and $\mathrm{Xu}$ [19] established the TOPSIS approach in a Pythagorean fuzzy environment and used it to assess the efficiency of private airline services.

Hesitancy is a natural phenomenon in the universe. Identifying the better alternatives having the same characteristics in daily life is complicated. Due to the uncertainty and hesitancy of the results, professional experts are experiencing difficulty in DM. To tackle hesitancy, Torra [20] developed the concept of hesitant FS (HFS). The HFS can be used to solve a variety of DM problems. Many authors used HFS to solve issues by aggregating operators (AOPs) in group DM (for detailed information, see [21-24]). Liao and $\mathrm{Xu}$ [25] identified generalized forms of the HF hybrid weighted averaging (HFHWA) operator, the HF hybrid weighted geometric (HFHWG) operator, generalized form of the quasi-HFHWA operator, and the generalized form of the quasi-HFHWG. Khan et al. [26] introduced the concept of Pythagorean HFS (PyHFS). They established an evaluation method and identified operators for data aggregation. $\mathrm{Xu}$ and Zhou [27] identified a novel concept of probabilistic $\mathrm{HF}$ sets (PHFSs). Inspired by the power of PHFSs, researchers extensively investigated the idea of multiattribute decision-making (MADM) (see [28-30] for detailed information). Yager [31] established a new idea called $q$-rung orthopair FSs (q-ROFSs), in which the number of the qth exponent of support for membership and the $q$ th exponent of support nonmembership is restricted to one, i.e., $\left(\beta_{\mathrm{F}}(x)\right)^{q}+\left(\psi_{\mathrm{F}}(x)\right)^{q} \leq 1$, and demonstrated that the $q$-ROFS is more general than the IFS and PFS. The $q$-ROFSs provide a broader range of fuzzy information and are the versatile and appropriate approach to deal with unpredictable situations. Yager and Alajlan [32] explored the fundamental properties of these $q$-ROFSs and discussed how they can be used in information representation. Subsequently, the authors in [33] put forward the notation of $q$-rung orthopair HF set ( $q$ ROHFS) and explored the operational laws which exist for any two $q$-ROHFSs. Wang et al. [34] investigated the Heronian mean operators in MADM in a $q$-ROHFS framework. They also proposed the Hamacher norm-based AOPs under dual hesitant $q$-ROFSs and discussed their usefulness in DM problems. Wang et al. [35] established the AOPs based on Muirhead mean under dual hesitant $q$-rung orthopair fuzzy information. Hussain and Yang [36] measured the entropy for HF information using the Hausdorff metric and the structure of HF TOPSIS. The TOPSIS is a valuable information analysis tool developed by Hwang and Yoon [37]; it is also known as the approximate ideal solution. It investigates the appropriate approach in terms of relative closeness based on their distances from the positive ideal solution (PIS) and the negative ideal solution (NIS), ensuring that the shortest distance from the PIS and the farthest distance from the NIS are satisfied. This analysis method effectively eliminates decision information uncertainty while maintaining the validity and precision of decision-making by simply measuring the distance between PIS and NIS and ranking them accordingly. TOPSIS method is straightforward and simple to understand and analyze as compared to the ELECTRE method, VIKOR method, and other conventional methods, so it has been extensively studied and implemented by researchers.

In recent years, several authors have presented TOPSIS in various fuzzy information. For example, Boran et al. [38] used TOPSIS to identify the best supplier by using IF information. Chen and Tsao [39] suggested the TOPSIS technique based on interval-valued fuzzy information and addressed the experimental results. The authors in [40] established the extended TOPSIS method for $q$-ROHFSs and addressed their significance in DM. Li [41] proposed a TOPSIS-based nonlinear programming technique for MADM with interval-valued IFs in order to deal with uncertainty in real-world DM problems. The TOPSIS model for $\mathrm{DM}$ problems in interval-valued IF information was introduced by Park et al. [42]. The Dombi-based AOPs for PF information is formulated in [43]. Barukab et al. [44] 


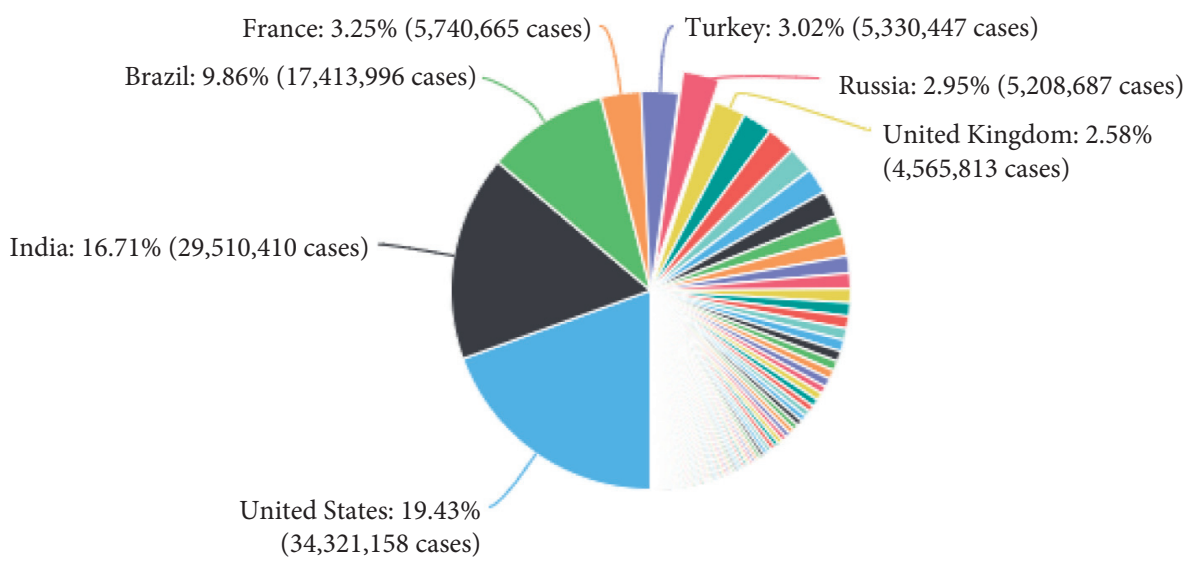

FIgURE 1: Distribution of cases all over the world. Source: Worldometer [2].

proposed the extended fuzzy TOPSIS method for spherical fuzzy information, which is based on the entropy measure. The aforesaid approach has been used by many other researchers; see [45-47] for more information. However, there are many research findings in applying the fuzzy TOPSIS method to solve MADM problems; the decision information used by these approaches is too old and restricted to manage increasingly challenging decision environments.

Pawlak [48] was the founder of exploring the dominating concept of rough set (RS) theory. The classical set theory which deals with inconsistent and imprecise information is extended by rough set theory. Recently, research on the rough set has progressed significantly, both in terms of theoretical implementations and theory itself. In recent decades, research has demonstrated the TOPSIS technique in a number of RS information. Su et al. [49] studied RS theory based on fuzzy TOPSIS on the serious game design assessment procedure. Khan et al. [50] implemented a rough set strategy and the TOPSIS method for selection of sites for food distribution. Lu and Zhao [51] investigated the improved TOPSIS method based on RS theory for selection of suppliers. A $b$-rough set model and its applications to DM using the TOPSIS approach have been discussed in [52]. The concept of RS has been expanded by several researchers around the world in different directions. Using the fuzzy relation rather than the crisp binary relation, Dubois and Prade [53] initiated the notion of fuzzy rough sets (FRSs). The hybrid structure of IFSs and RS, intuitionistic RS (IFR set), was introduced by Cornelis et al. [54]. Zhou and $\mathrm{Wu}$ [55] established a novel DM technique under the IFR environment to address their constructive and axiomatic analysis in detail by utilizing IFR approximations. Zhan et al. [56] presented the DM methodology under the IFR environment and explored their applications in real-world problems. Different extensions of the IFRS are being investigated $[57,58]$ to tackle the uncertainty in MCGDM problems. Chinram et al. [59] established the algebraic norm-based AOPs based on the EDAS technique under IFR information and discussed their applications in MAGDM.

In some real-life circumstances, there exist numerous cases when decision makers (DMs) have their strong points of view about ranking or rating of plans, projects, or political statements of a government. For example, let the administration of a university start megaprojects of the football ground to render his accomplishment and performance. The members of the university administration may rate their project highly by assigning positive membership $=0.9$, whereas the others may rate the same project as a wastage of money and try to defame it by providing strongly opposite points of view. So, they assign negative membership $=0.7$. In this situation, their sum $0.9+0.7>1$ and $(0.9)^{2}+(0.7)^{2}>1$ but $(0.9)^{q}+(0.7)^{q}<1$ for $q>3$ so that it is neither IFN nor PFN but it is $q$-ROFN. Thus, Yager's $q$-ROFNs are efficient to deal with vagueness in the data. $q$-rung orthopair probabilistic hesitant fuzzy rough set (q-ROHFRS), a hybrid intelligent structure of rough sets and $q$-ROPHFS, is an advanced classification strategy that has attracted researchers to address ambiguous and incomplete data. From the analysis, it is concluded that, in decision-making, AOP plays a significant role in aggregating the collective data from different sources to a single value. In accordance with the best available knowledge to date, the development of the AOP with the hybridization of the $q$-ROPHFS with a rough set is not observed in the $q$ ROF setting. As a result, the current $q$-ROPHF rough structure is inspired, and we define a list of Einstein aggregation operators depending on rough data, such as $q$ rung orthopair probabilistic hesitant fuzzy Einstein weighted averaging, Einstein ordered weighted averaging, Einstein hybrid weighted averaging, Einstein weighted geometric, Einstein ordered weighted geometric, and Einstein hybrid weighted geometric aggregation operators, under the Einstein $t$-norm and $s$-norm.

The description of the main objectives of the present work is as follows:

(1) To introduce a novel idea of $q$-rung orthopair probabilistic hesitant fuzzy rough sets ( $q$ ROPHFRSs) and investigate their basic operational laws.

(2) Establish a list of AOPs based on Einstein $t$-norm and $t$-conorm and comprehensively explore the relevant properties. 
(3) To develop a DM strategy for aggregating unpredictability in real-world DM problems employing suggested aggregation operators.

(4) In addition, a case study of drug selection for mild COVID-19 symptoms is described to demonstrate the applicability and utility of the established operators.

(5) Finally, a comparison with the $q$-ROPHFR-TOPSIS method is made to interpret the outcomes. The ranking of the obtained results is presented graphically.

\section{Basic Terminologies}

This section covers a variety of significant and fundamental concepts, i.e., fuzzy set (FS), intuitionistic FS (IFS), q-rung orthopair FS (q-ROFS), hesitant FS (HFS), $q$-rung orthopair HFS (q-ROHFS), $q$-rung orthopair probabilistic HFS ( $q$-ROPHFS), rough sets (RSs), and $q$-rung orthopair FRS (q-ROFRS).

Definition 1. (see [15]). For a universal set $\Omega$, an FS $Q$ is presented as

$$
Q=\left\{\left\langle x, \beta_{Q}(x)\right\rangle \mid x \in F\right\}
$$

for each $x \in F$, and the function $\beta_{Q}(x)$ belongs to $[0,1]$ that represent the degree of membership.

Definition 2. (see [16]). For a universal set $\Omega$, an IFS $F$ over $\Omega$ is described as

$$
\mathrm{F}=\left\{\left\langle x, \beta_{\mathrm{F}}(x), \psi_{\mathrm{F}}(x)\right\rangle \mid x \in \Omega\right\} .
$$

For each $x \in \mathrm{F}$, the functions $\left.\beta_{\mathrm{F}}: \Omega \longrightarrow 0,1\right]$ and $\left.\psi_{\mathrm{F}}: \Omega \longrightarrow 0,1\right]$ represent the membership and nonmembership, respectively, which must satisfy the property $0 \leq \beta_{\mathrm{F}}(x)+\psi_{\mathrm{F}}(x) \leq 1$.

Definition 3. (see [60]). For a universal set $\Omega$, an HFS $A$ in $\Omega$ is represented mathematically as

$$
A=\left\{\left\langle x, \beta_{h_{A}}(x)\right\rangle \mid x \in \Omega\right\},
$$

where $\beta_{h_{A}}(x)$ is a set of some values in $[0,1]$ representing the degree of membership for the element $x \in \Omega$ of the set $A$.

Definition 4. For a universal set $\Omega$, a probabilistic HF set (PHFS) $P$ in $\Omega$ is described mathematically as

$$
P=\left\{\left\langle x, \beta_{h_{P}}(x) / \partial_{h(x)}\right\rangle \mid x \in \Omega\right\},
$$

where $\beta_{h_{P}}(x)$ is a subset of $[0,1]$ and $\beta_{h_{P}}(x) / \partial_{x}$ shows a membership grade of the element $x \in \Omega$ to the set $P$. And $\partial_{x}$ shows the possibilities with the property that $\oplus_{t=1}^{s} \partial_{h_{t}}=1$.

Definition 5. (see [31]). For a universal set $\Omega$, a $q$-ROFS $F$ over $\Omega$ is defined as

$$
\mathrm{F}=\left\{\left\langle x, \beta_{\mathrm{F}}(x), \psi_{\mathrm{F}}(x)\right\rangle \mid x \in \Omega\right\},
$$

for each $x \in \mathrm{F}$; the functions $\left.\beta_{\mathrm{F}}: \Omega \longrightarrow 0,1\right]$ and $\left.\psi_{\mathrm{F}}: \Omega \longrightarrow 0,1\right]$ denote the membership and nonmembership, respectively, which must satisfy $\left(\psi_{\mathrm{F}}(x)\right)^{q}+\left(\beta_{\mathrm{F}}(x)\right)^{q} \leq 1,(q>2 \in \mathbb{Z})$ (Figure 2).

Definition 6. (see [33]). For a universal set $\Omega$, the mathematical representation of $q$-ROHFS $\sqsupset$ is as follows:

$$
\beth=\left\{x, \beta_{h_{\sqsupset}}(x), \psi_{h_{\sqsupset}}(x) \mid x \in \Omega\right\},
$$

where $\beta_{h_{\beth}}(x)$ and $\psi_{h_{7}}(x)$ are sets of some values in $[0,1]$. It is required to satisfy the following properties: $\forall x \in \Omega$, $\forall \Phi_{\beth}(x) \in \beta_{h_{\beth}}(x), \forall \nu_{\beth}(x) \in \psi_{h_{\beth}}(x)$ with $\left(\max \left(\beta_{h_{\beth}}(x)\right)\right)^{q}+$ $\left(\min \left(\psi_{h_{\sqsupset}}(x)\right)\right)^{q} \leq 1 \quad$ and $\quad\left(\min \left(\beta_{h_{\sqsupset}}(x)\right)\right)^{q}+(\max$ $\left.\left(\psi_{h_{\beth}}(x)\right)\right)^{q} \leq 1$. For simplicity, we will use a pair $\sqsupset=$ $\left(\beta_{h_{\beth}}, \psi_{h_{\beth}}\right)$ to mean $q$-ROHF number $(q$-ROHFN).

Definition 7. (see [33]). Let $\beth_{1}=\left(\beta_{h_{\sqsupset}}, \psi_{h_{\sqsupset}}\right)$ and $\beth_{2}=\left(\beta_{h_{2}}, \psi_{h_{\beth}}\right)$ be two $q$-ROHFNs. Then, the basic set theoretic operations are as follows:

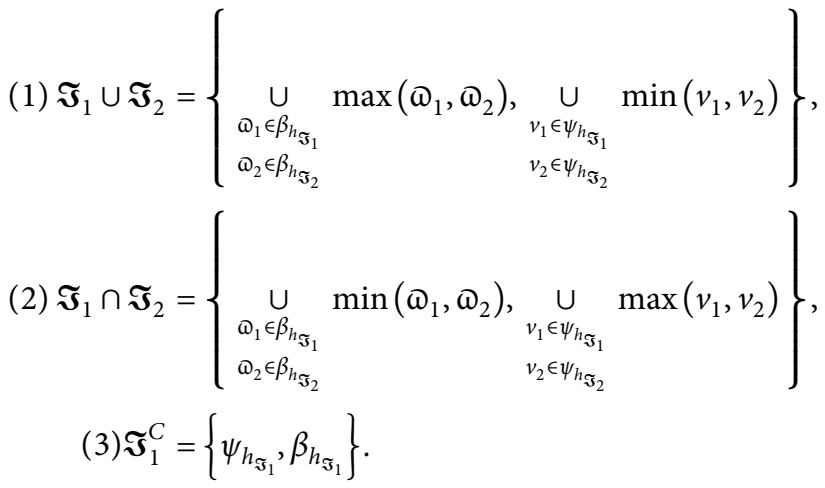

Definition 8. Let $\mathfrak{J}_{1}=\left(\beta_{h_{\mathfrak{\Im}_{1}}}, \psi_{h_{\mathfrak{\Im}_{1}}}\right)$ and $\mathfrak{J}_{2}=\left(\beta_{h_{\mathfrak{s}_{2}}}, \psi_{h_{\mathfrak{\Im}_{2}}}\right)$ be two $q$-ROHFNs where $q>2$ and $\gamma>0$ are any real number. Then, the operational laws based on Einstein $t$-norm and $t$ conorm can be defined as 


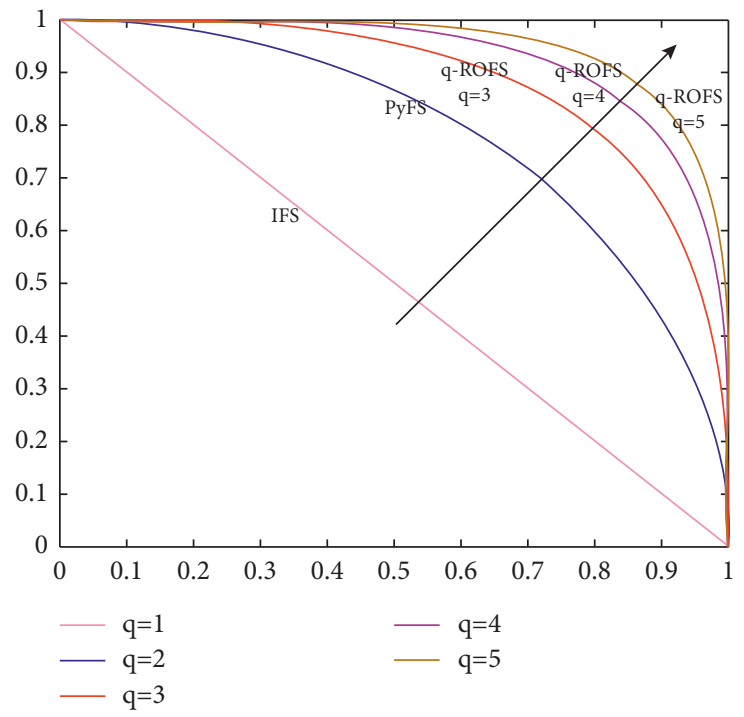

Figure 2: Geometrical representation of IFS, PyFs, and $q$-ROFSs $(q=1-5)$.

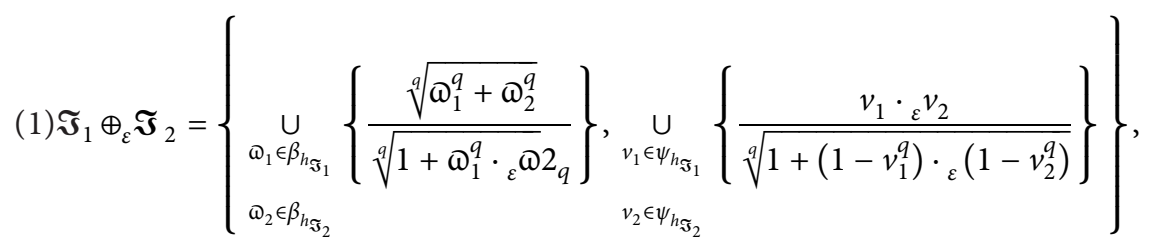

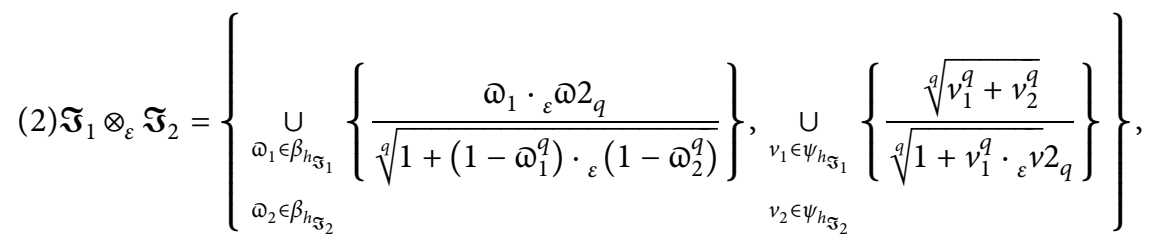

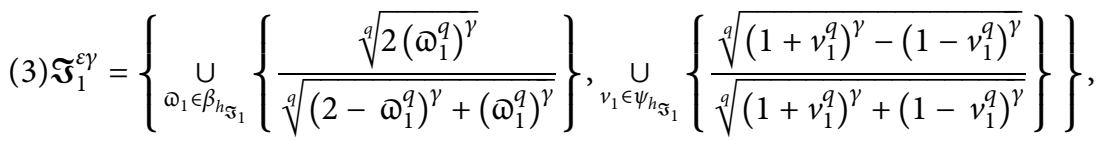

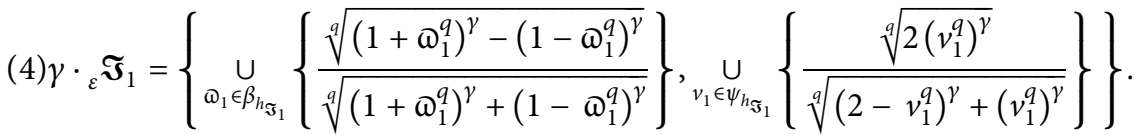

Definition 9. For a universal set $\Omega$, a $q$-ROPHFS $\Im$ is defined as

$$
\mathfrak{I}=\left\{\left\langle x, \frac{\beta_{h_{\mathfrak{J}}}(x)}{\partial_{x}}, \frac{\psi_{h_{\mathfrak{J}}}(x)}{\partial_{x}}\right\rangle \mid x \in \Omega\right\},
$$

where $\beta_{h_{\Im}}(x) / \partial_{x}$ and $\psi_{h_{\Im}}(x) / \partial_{x}$ are sets of some values in $[0,1]$ which denote the membership and nonmembership, respectively. $\partial_{x}$ and $\partial_{x}$ represent the possibilities of membership and nonmembership with the following property: $0 \leq \partial_{h_{t}}$ and $\partial_{h_{t}} \leq 1$ with $\oplus_{i=1}^{p} \partial_{h_{t}} \leq 1$ and $\oplus_{i=1}^{p} \partial_{h_{t}} \leq 1 \quad(p$ represents that the total elements exist in the $q$-ROPHFS). It is required to satisfy the following properties: $\forall x \in \Omega$, $\forall \omega_{\mathfrak{J}}(x) \in \quad \beta_{h_{\mathfrak{S}}}(x), \quad$ and $\quad \forall v_{\mathfrak{J}}(x) \in \psi_{h_{\mathfrak{S}}}(x) \quad$ with $\left(\max \left(\beta_{h_{\mathfrak{S}}}(x)\right)\right)^{q_{+}} \quad\left(\min \left(\psi_{h_{\mathfrak{S}}}(x)\right)\right)^{q} \leq 1 \quad$ and $\left(\min \left(\beta_{h_{\mathfrak{S}}}(x)\right)\right)^{q}+\left(\max \left(\psi_{h_{\mathfrak{S}}}(x)\right)\right)^{q} \leq 1$. For simplicity, we will use a pair $\mathfrak{I}=\left(\beta_{h_{\mathfrak{S}}} / \partial_{x}, \psi_{h_{\mathfrak{S}}} / \partial_{x}\right)$ to mean a $q$-ROPHF number (q-ROPHFN).

Definition 10. Let $\mathfrak{\Im}_{1}=\left(\beta_{h_{\mathfrak{\Im}}} / ð_{x_{1}}, \psi_{h_{\mathfrak{I}}} / \partial_{x_{1}}\right)$ and $\mathfrak{\Im}_{2}=\left(\beta_{{\mathfrak{\Im}_{2}}_{2}} / \partial_{x_{2}}, \psi_{h_{\mathfrak{\Im}_{2}}} / \partial_{x_{2}}\right)$ be two q-ROPHFNs. Then, the basic set theoretic operations are as follows: 


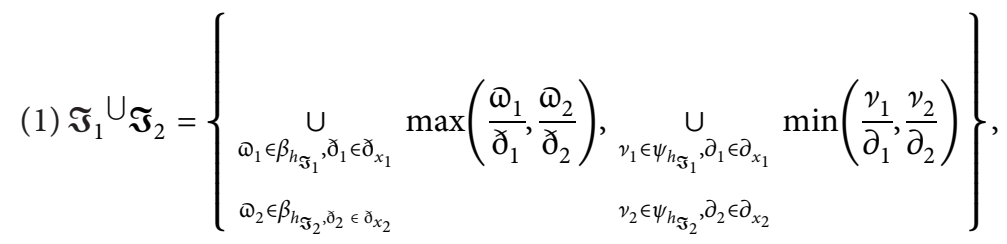

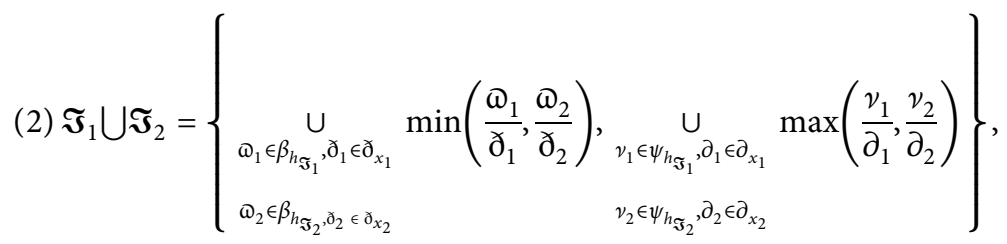

$$
\begin{aligned}
& \text { (3) } \mathfrak{\Im}_{1}^{c}=\left\{\frac{\psi_{h_{\mathfrak{F}_{1}}}}{\partial_{1}}, \frac{\beta_{{\mathfrak{\Im}_{1}}_{1}}}{\partial_{1}}\right\} \text {. }
\end{aligned}
$$

Definition

$\mathfrak{I}_{1}=\left(\beta_{h_{\mathfrak{I}_{1}}} / \partial_{1}, \psi_{h_{\mathfrak{\Im}_{1}}} / \partial_{1}\right)$ and $\mathfrak{I}_{2}=\left(\beta_{h_{\mathfrak{\Im}_{2}}} / \partial_{2}, \psi_{h_{\mathfrak{J}_{2}}} / \partial_{2}\right)$ be two $q-$
Then, the operational laws based on Einstein $t$-norm and $t$ conorm can be defined as

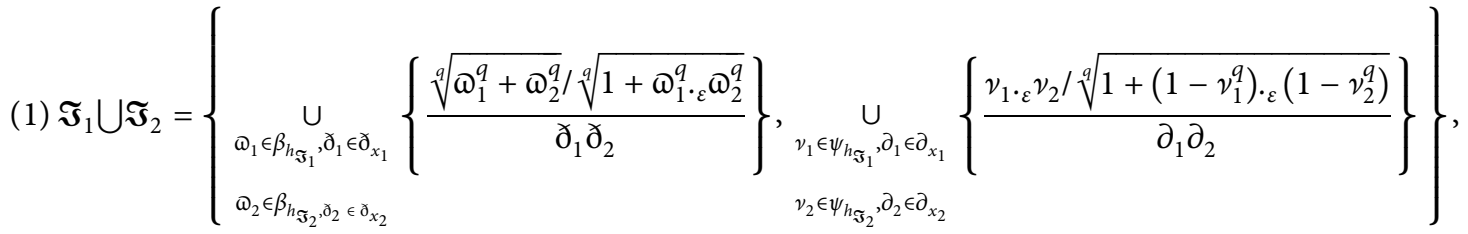

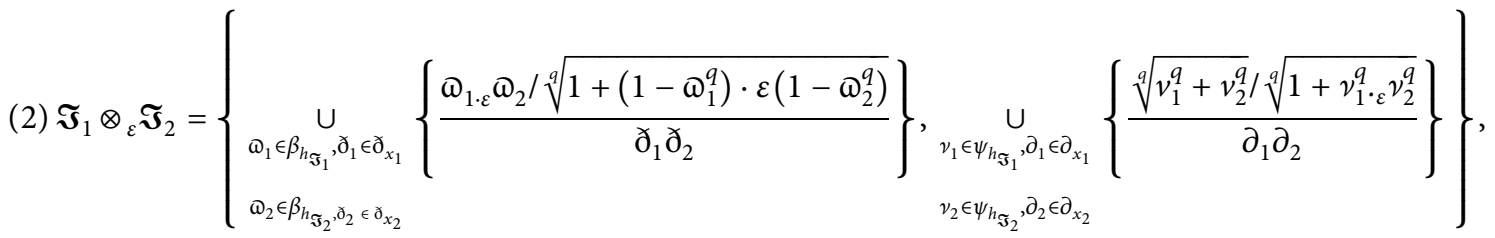

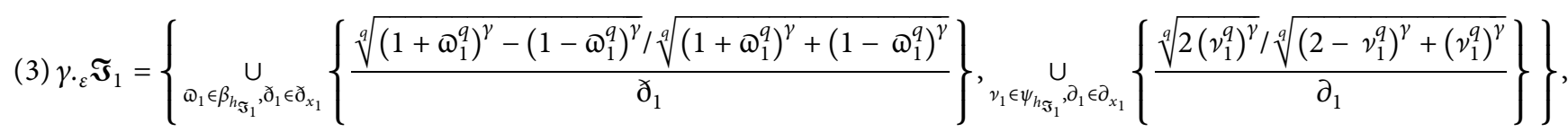

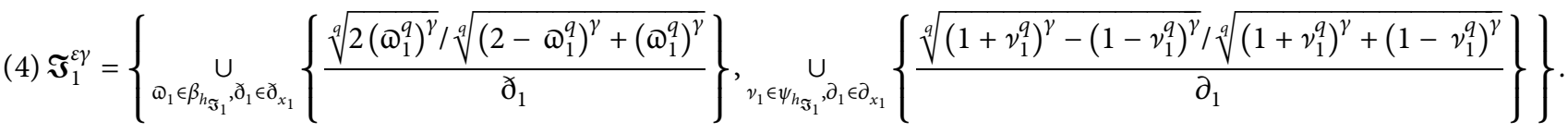

Definition 12. Let $\Omega$ be the universal set and $\aleph \subseteq \Omega \times \Omega$ be a (crisp) relation. Then,

(1) $\aleph$ is reflexive if $(\stackrel{\hat{g}}{\cdot}, \hat{g}) \in \aleph$, for each $\stackrel{\wedge}{g} \in \Omega$

(3) $\aleph$ is symmetric if $\forall \hat{g}, a \in \Omega$ and $(\stackrel{\wedge}{g}, a) \in \aleph$, then $(a, \hat{g}) \in \aleph$

(4) $\aleph$ is transitive if $\forall \hat{g}, a, b \in \Omega,(\hat{g}, a) \in \Omega$, and $(a, b) \in \aleph$ implies $(\stackrel{\wedge}{g}, b) \in \mathcal{N}$
Definition 13. (see [48]). Let $\Omega$ be a universal set and $\aleph$ be any relation on $\Omega$. Define a set-valued mapping $\stackrel{\aleph}{*}^{*}: \Omega \longrightarrow M(\Omega)$ by $\aleph^{*}(\stackrel{\wedge}{g})=\{a \in \Omega \mid(\hat{g} \cdot a) \in \mathcal{N}\}$, for $\hat{g} \in \Omega$ where $\aleph^{*}(\hat{g})$ is called a successor neighborhood of the element $\hat{g}$ with respect to relation $\aleph$. The pair $(\Omega, \aleph)$ is called the (crisp) approximation space. Now, for any set $b \subseteq \Omega$, the lower and upper approximation of $b$ with respect to the approximation space $(\Omega, \aleph)$ is defined as 


$$
\begin{aligned}
& \underline{\aleph}(b)=\left\{\hat{g} \in \Omega \mid \aleph^{*}\left(\begin{array}{l}
\wedge \\
g
\end{array}\right) \subseteq b\right\}, \\
& \bar{\aleph}(b)=\left\{\hat{g} \in \Omega \mid \aleph^{*}\left(\begin{array}{l}
\wedge \\
g
\end{array}\right) \cap b \neq \phi\right\} .
\end{aligned}
$$

The pair $(\underline{\aleph}(b), \bar{\aleph}(b))$ is called the rough set, and both $\underline{\underline{N}}(b), \bar{N}(b): M(\Omega) \longrightarrow M(\Omega)$ are upper and lower approximation operators.

Definition 14. (see [59]). Let $\Omega$ be the universal set and $\aleph \in(\Omega \times \Omega)$ be an intuitionistic fuzzy relation. Then,

(1) $\aleph$ is reflexive if $\emptyset_{\aleph}(\hat{g}, \hat{g})=1 \quad$ and $\nu_{\aleph}(\stackrel{\wedge}{g}, \stackrel{\wedge}{g})=0, \forall \hat{g} \in \Omega$,

(2) $\aleph$ is symmetric if $\forall(\hat{g}, a) \in \Omega \times \Omega$, $\varpi_{\mathbb{N}}(\hat{g}, a)=\Phi_{\aleph}(a, \hat{g})$ and $\nu_{\aleph}(\hat{g}, a)=\nu_{\aleph}(a, \hat{g})$,

(3) $\aleph$ is transitive if $\forall(\hat{g}, b) \in \Omega \times \Omega$,

$$
\begin{aligned}
& \varpi_{\aleph}(\hat{g}, b)=\underset{a \in \Omega}{\vee}\left[\Phi_{\aleph}(\hat{g}, a) \wedge \varpi_{\aleph}(a, b)\right], \\
& \nu_{\aleph}(\hat{g}, b)=\wedge_{a \in \Omega}\left[\nu_{\aleph}(\hat{g}, a) \wedge \nu_{\aleph}(a, b)\right] .
\end{aligned}
$$

Definition 15. Let $\Omega$ be the universal set. Then, any $\aleph \in q-$ $\operatorname{RFS}(\Omega \times \Omega)$ is called a $q$-rung relation. The pair $(\Omega, \aleph)$ is said to be $q$-rung approximation space. Now, for any $b \subseteq q-\operatorname{RFS}(\Omega)$, the upper and lower approximations of $b$ with respect to the $q$-RF approximation space $(\Omega, \aleph)$ are two
$q$-RFSs, which are denoted by $\bar{\aleph}(b)$ and $\underline{\aleph}(b)$ and are defined as

$$
\begin{aligned}
& \bar{\aleph}(b)=\left\{\hat{g}, \Phi_{\bar{\aleph}(b)}(\hat{g} \cdot), \nu_{\bar{\aleph}(b)}(\hat{g}) \mid \hat{g} \in \Omega\right\}, \\
& \underline{\aleph}(b)=\left\{\hat{g}, \Phi_{\underline{\underline{N}(b)}}(\hat{g} \cdot), \nu_{\underline{\aleph}(b)}(\hat{g}) \mid \hat{g} \in \Omega\right\},
\end{aligned}
$$

where

$$
\begin{aligned}
& \Phi_{\bar{\aleph}(b)}(\stackrel{\wedge}{g})=\underset{g \in \Omega}{\vee}\left[\Phi_{\aleph}(\stackrel{\wedge}{g}, g) \vee \Phi_{b}(\stackrel{\wedge}{g})\right], \\
& \nu_{\bar{\aleph}(b)}(\stackrel{\wedge}{g})=\wedge_{g \in \Omega}\left[\nu_{\aleph}(\stackrel{\wedge}{g}, c) \wedge \nu_{b}(\stackrel{\wedge}{g})\right],
\end{aligned}
$$

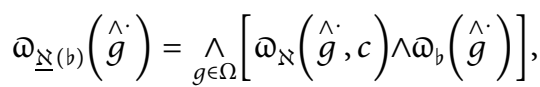

$$
\begin{aligned}
& \nu_{\underline{\aleph}(b)}(\stackrel{\wedge}{g})=\underset{g \in \Omega}{\vee}\left[\nu_{\aleph}(\stackrel{\wedge}{g}, c) \vee \nu_{b}(\stackrel{\wedge}{g})\right] \text {, }
\end{aligned}
$$

such that

$$
\begin{aligned}
& \left.0 \leq\left(\left(\Phi_{\bar{\aleph}(b)}(\hat{g})\right)\right)^{q}+\left(\nu_{\bar{\aleph}(b)}(\hat{g} \cdot)\right)^{q}\right)^{1 / q} \leq 1, \\
& 0 \leq\left(\left(\Phi_{\underline{\underline{\aleph}}(b)}(\hat{g} \cdot)\right)^{q}+\left(\nu_{\underline{\underline{\aleph}}(b)}(\hat{g} \cdot)\right)^{q}\right)^{1 / q} \leq 1 .
\end{aligned}
$$

As $\quad(\underline{N}(b), \bar{N}(b)) \quad$ are $\quad q$-RFSs, $\underline{\text { N(b) }}$, $\bar{\aleph}(b): q-\operatorname{RFS}(\Omega) \longrightarrow q-\operatorname{RFS}(\Omega)$ are upper and lower approximation operators. The pair

$$
\aleph(b)=(\underline{\aleph}(b), \bar{\aleph}(b))=\left\{\hat{g} \cdot\left(\Phi_{\underline{\aleph}(b)}(\hat{g} \cdot), \nu_{\underline{\aleph}(b)}(\stackrel{\wedge}{g}),\left(\Phi_{\bar{\aleph}(b)}(\hat{g} \cdot), \nu_{\bar{\aleph}(b)}(\hat{g} \cdot)\right) \mid \hat{g} \cdot \in b\right\}\right.
$$

is known as the q-rung rough set. For simplicity,

$\aleph(b)=\left\{\hat{g}, \Phi_{\underline{\underline{N}(b)}}(\hat{g} \cdot), \nu_{\underline{\underline{\aleph}}(b)}(\hat{g} \cdot),\left(\varpi_{\bar{\aleph}(b)}(\hat{g} \cdot), \nu_{\bar{\aleph}(b)}(\hat{g})\right) \mid \hat{g} \in \Omega\right\}$

is represented as $\aleph(b)=((\underline{\varrho}, \underline{v}),(\bar{\emptyset}, \bar{\nu}))$ and is known as $q$ RFRV.

\section{Construction of $q$-Rung Orthopair Hesitant Fuzzy Rough Sets}

In this section, we propose the notion of $q$-ROHFRS which is the hybrid structure of the rough set and $q$-ROFS. We also introduce the new accuracy and score functions to rank the $q$-ROHFRS and also put forward its basic operational laws.

Definition 16. Let $\Omega$ be the universal set. Then, any subset $\aleph \in q-\operatorname{ROHFS}(\Omega \times \Omega)$ is said to be a $q$-RHF relation. The pair $(\Omega, \aleph)$ is called the $q$-ROHF approximation space. If for any $b \subseteq q-\operatorname{ROHFS}(\Omega)$, then the upper and lower approximations of $b$ with respect to the $q$-ROHF approximation space $(\Omega, \aleph)$ are two $q$-ROHFSs, which are denoted by $\bar{\aleph}(b)$ and $\underline{\aleph}(b)$ and defined as

$$
\begin{aligned}
& \underline{\aleph}(b)=\left\{\hat{g}, \beta_{h_{\underline{\underline{\underline{x}}}(b)}}(\hat{g}), \psi_{h_{\underline{\underline{\underline{X}}}(b)}}(\hat{g}) \mid \hat{g} \cdot \in\right\}, \\
& \bar{\aleph}(b)=\left\{\hat{g}, \beta_{h_{\overline{\mathrm{N}}(b)}}(\hat{g}), \psi_{h_{\overline{\mathrm{N}}(b)}(\hat{g}) \mid \hat{g} \in} \in \Omega\right\},
\end{aligned}
$$

where

$$
\begin{aligned}
& \beta_{h_{\underline{\underline{X}}(0)}}(\hat{g})={ }_{k \in \Omega}\left[\beta_{h_{\mathbb{N}}}(\hat{g}, k) \wedge \beta_{h_{\underline{b}}}(k)\right], \\
& \psi_{h_{\underline{\underline{X}}(b)}}(\stackrel{\wedge}{g})=\underset{k \in \Omega}{\vee}\left[\psi_{h_{\mathbb{N}}}(\stackrel{\wedge}{g}, k) \vee \psi_{h_{b}}(k)\right], \\
& \beta_{h_{\underline{\underline{X}}(\mathfrak{b})}}(\hat{g})=\underset{k \in \Omega}{\vee}\left[\beta_{h_{\mathfrak{X}}}(\hat{g}, k) \vee \beta_{h_{\mathfrak{o}}}(k)\right], \\
& \psi_{h_{\underline{\underline{X}}(b)}}(\stackrel{\wedge}{g})=\wedge \wedge_{k \in \Omega}\left[\psi_{h_{\mathbb{N}}}(\stackrel{\wedge}{g}, k) \wedge \psi_{h_{b}}(k)\right],
\end{aligned}
$$

such that

$$
\begin{aligned}
& 0 \leq\left(\max \left(\beta_{h_{\overline{\mathbb{N}}(b)}}(\stackrel{\wedge}{g})\right)\right)^{q}+\left(\min \left(\psi_{h_{\overline{\mathbb{N}}(b)}}(\stackrel{\wedge}{g})\right)\right)^{q} \leq 1, \\
& 0 \leq\left(\min \left(\beta_{h_{\underline{\underline{\underline{K}}}(b)}}(\stackrel{\wedge}{g})\right)^{q}+\left(\max \left(\psi_{h_{\underline{\underline{N}}(b)}}(\stackrel{\wedge}{g})\right)\right)^{q} \leq 1 .\right.
\end{aligned}
$$

As

$(\bar{\aleph}(b), \underline{N}(b))$

are

$q$-ROHFSs, $\bar{\aleph}(b), \underline{\aleph}(b): q-\operatorname{ROHFS}(\Omega) \longrightarrow q-\operatorname{RFS}(\Omega)$ are upper and lower approximation operators. The pair 


$$
\aleph(b)=(\underline{\aleph}(b), \bar{\aleph}(b))=\left\{\hat{g} \cdot\left(\beta_{h_{\underline{\aleph}(b)}}(\stackrel{\wedge}{g}), \psi_{h_{\underline{\aleph}(b)}}(\stackrel{\wedge}{g})\right),\left(\beta_{h_{\bar{\aleph}(b)}}(\stackrel{\wedge}{g}), \psi_{h_{\bar{\aleph}(b)}}(\stackrel{\wedge}{g})\right) \mid \hat{g} \in b\right\}
$$

will be called $q$-ROHFRS. For simplicity,

$$
\aleph(b)=\left\{\stackrel{\wedge}{g},\left(\beta_{h_{\underline{\aleph}(b)}}(\stackrel{\wedge}{g}), \psi_{h_{\underline{\aleph}(b)}}(\stackrel{\wedge}{g})\right),\left(\beta_{h_{\bar{\aleph}(b)}}(\stackrel{\wedge}{g}), \psi_{h_{\bar{\aleph}(b)}}(\stackrel{\wedge}{g})\right) \mid \hat{g} \in b\right\}
$$

is represented as $\aleph(b)=((\underline{\xi}, \eta),(\bar{\beta}, \bar{\psi}))$ and is known as $q$ ROHFRV.

Definition 17. Let $\aleph\left(b_{1}\right)=\left(\underline{\aleph}\left(b_{1}\right), \bar{\aleph}\left(b_{1}\right)\right) \quad$ and $\aleph\left(b_{2}\right)=\left(\underline{\aleph}\left(b_{2}\right), \bar{\aleph}\left(b_{2}\right)\right)$ be two $q$-ROHFRSs. Then,

(1) $\aleph\left(b_{1}\right) \cup \aleph\left(b_{2}\right)=\left\{\left(\underline{\aleph}\left(b_{1}\right) \cup \underline{\aleph}\left(b_{2}\right)\right)\right.$, $\left.\left(\bar{\aleph}\left(b_{1}\right) \cup \bar{\aleph}\left(b_{2}\right)\right)\right\}$

(2) $\aleph\left(b_{1}\right) \cap \aleph\left(b_{2}\right)=\left\{\left(\underline{\aleph}\left(b_{1}\right) \cap \underline{\aleph}\left(b_{2}\right)\right)\right.$, $\left.\left(\bar{\aleph}\left(b_{1}\right) \cap \bar{\aleph}\left(b_{2}\right)\right)\right\}$

(3) $\aleph\left(b_{1}\right) \oplus \aleph\left(b_{2}\right)=\left\{\left(\underline{\aleph}\left(b_{1}\right) \oplus \underline{\aleph}\left(b_{2}\right)\right),\left(\bar{\aleph}\left(b_{1}\right) \oplus \bar{\aleph}\left(b_{2}\right)\right)\right\}$

(4) $\aleph\left(b_{1}\right) \otimes \aleph\left(b_{2}\right)=\left\{\left(\underline{\aleph}\left(b_{1}\right) \otimes \underline{\aleph}\left(b_{2}\right)\right)\right.$, $\left.\left(\bar{\aleph}\left(b_{1}\right) \otimes \bar{\aleph}\left(b_{2}\right)\right)\right\}$
(5) $\aleph\left(b_{1}\right) \subseteq \aleph\left(b_{2}\right)=\left\{\left(\underline{\aleph}\left(b_{1}\right) \subseteq \underline{\aleph}\left(b_{2}\right)\right)\right.$ and $\left.\left(\bar{\aleph}\left(b_{1}\right) \subseteq \bar{\aleph}\left(b_{2}\right)\right)\right\}$

(6) $\gamma \aleph\left(b_{1}\right)=\left(\gamma \underline{\aleph}\left(b_{1}\right), \gamma \bar{\aleph}\left(b_{1}\right)\right)$ for $\gamma \geq 1$

(7) $\left(\aleph\left(b_{1}\right)\right)^{\gamma}=\left(\left(\left(\underline{\aleph}\left(b_{1}\right)\right)^{\gamma},\left(\bar{\aleph}\left(b_{1}\right)\right)^{\gamma}\right)\right)$ for $\gamma \geq 1$

(8) $\aleph\left(b_{1}\right)^{c}=\left(\underline{\aleph}\left(b_{1}\right)^{c}, \bar{\aleph}\left(b_{1}\right)^{c}\right) \quad$ where $\aleph\left(b_{1}\right)^{c}$ and $\bar{\aleph}\left(b_{1}\right)^{c}$ show the complement of $q$-rung fuzzy rough approximation operators $\aleph\left(b_{1}\right)$ and $\bar{\aleph}\left(b_{1}\right)$, which is $\underline{\underline{N}}\left(b_{1}\right)^{c}=\left(\psi_{h_{\aleph(b)}}, \beta_{h_{\aleph(b)}}\right)$

(9) $\aleph\left(b_{1}\right)=\aleph\left(b_{2}\right)$ iff $\underline{\aleph}\left(b_{1}\right)=\underline{\aleph}\left(b_{2}\right)$ and $\bar{\aleph}\left(b_{1}\right)=\bar{\aleph}\left(b_{2}\right)$

For comparing/ranking two or more q-ROHFRVs, the score function will be utilized, whereas the accuracy function will be used when the score values are equal. The accuracy function will be used when the score values are equal.

Definition 18. The score function for $q$-ROHFRV $\aleph(b)=$ $(\underline{\aleph}(b), \bar{\aleph}(b))=((\xi, \eta),(\bar{\beta}, \bar{\psi}))$ is given as

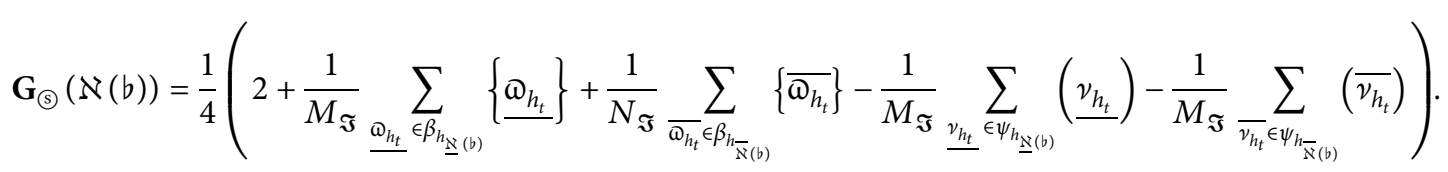

The accuracy function for $q$-ROHFRV $\aleph(b)=(\underline{\aleph}(b), \bar{\aleph}(b))=((\underline{\xi}, \underline{\eta}),(\bar{\beta}, t \bar{\psi}))$ is given as

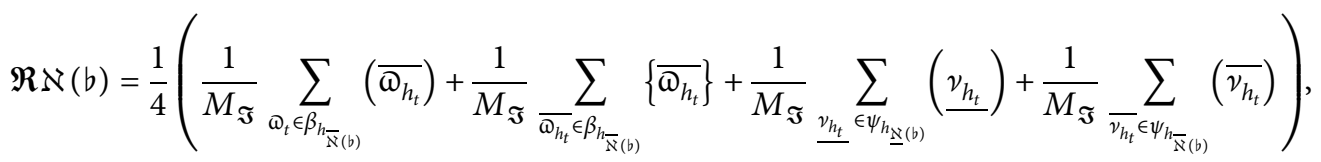

where $M_{\mathfrak{\Im}}$ and $N_{\mathfrak{\Im}}$ are the number of elements in $\beta_{h_{g}}$ and $\psi_{h_{g}}$, respectively.

Definition 19. Suppose $\aleph\left(b_{1}\right)=\left(\underline{\aleph}\left(b_{1}\right), \bar{\aleph}\left(b_{1}\right)\right)$ and $\aleph\left(b_{2}\right)=\left(\underline{\aleph}\left(b_{2}\right), \bar{\aleph}\left(b_{2}\right)\right)$ are two $q$-ROHFRVs. Then,

(1) If $\mathbf{G}_{\odot}\left(\aleph\left(b_{1}\right)\right)>\mathbf{G}_{\odot}\left(\aleph\left(b_{2}\right)\right)$, then $\aleph\left(b_{1}\right)>\aleph\left(b_{2}\right)$

(2) If $\mathbf{G}_{\odot}\left(\aleph\left(b_{1}\right)\right)<\mathbf{G}_{\odot}\left(\aleph\left(b_{2}\right)\right)$, then $\aleph\left(b_{1}\right)<\aleph\left(b_{2}\right)$

(3) If $\mathbf{G}_{\odot}\left(\aleph\left(b_{1}\right)\right)=\mathbf{G}_{\odot}\left(\aleph\left(b_{2}\right)\right)$, then

(a) If $\mathfrak{R}\left(\aleph\left(b_{1}\right)\right)>\Re\left(\aleph\left(b_{2}\right)\right)$, then $\aleph\left(b_{1}\right)>\aleph\left(b_{2}\right)$

(b) If $\Re\left(\aleph\left(b_{1}\right)\right)<\Re\left(\aleph\left(b_{2}\right)\right)$, then $\aleph\left(b_{1}\right)<\aleph\left(b_{2}\right)$

(c) If $\mathfrak{R}\left(\aleph\left(b_{1}\right)\right)=\mathfrak{R}\left(\aleph\left(b_{2}\right)\right)$, then $\aleph\left(b_{1}\right)=\aleph\left(b_{2}\right)$

\section{Construction of $q$-Rung Orthopair Probabilistic Hesitant Fuzzy Rough Sets}

This section deals with the notion of $q$-ROPHFRS which is the hybrid structure of the rough set and $q$-ROPHFS. We also establish the new score and accuracy functions to rank the $q$-ROPHFRS and also discuss the operational laws.

Definition 20. Let $\Omega$ be the universal set. Then, any subset $\aleph \in q-\operatorname{ROPHFS}(\Omega \times \Omega)$ is said to be a $q$-rung probabilistic HF relation. The pair $(\Omega, \aleph)$ is called the $q$-ROPHF approximation space. If for any $b \subseteq q-\operatorname{ROPHFS}(\Omega)$, the upper and lower approximations of $b$ with respect to the $q$-ROPHF approximation space $(\Omega, \aleph)$ are two $q$-ROPHFSs, which are denoted by $\bar{\aleph}(b)$ and $\underline{\aleph}(b)$ and defined as

$$
\begin{aligned}
& \bar{\aleph}(b)=\left\{\hat{g}, \frac{\beta_{h_{\overline{\mathrm{N}}(b)}}(\hat{g})}{\partial_{h_{\overline{\mathrm{N}}(b)}}}, \frac{\psi_{h_{\overline{\mathrm{K}}(b)}}(\hat{g})}{\partial_{h_{\overline{\mathrm{N}}(b)}}} \mid \hat{g} \in \Omega\right\}, \\
& \overline{\mathcal{N}}(b)=\left\{\hat{g}, \frac{\beta_{h_{\overline{\mathrm{N}}(b)}}(\hat{g})}{\partial_{h_{\overline{\mathrm{N}}(b)}}}, \frac{\psi_{h_{\overline{\mathrm{K}}(b)}}(\hat{g})}{\partial_{h_{\overline{\mathrm{N}}(b)}}} \mid \hat{g} \in \Omega\right\},
\end{aligned}
$$


where

$$
\begin{aligned}
& \frac{\beta_{h_{\overline{\mathrm{\aleph}}(b)}}(\stackrel{\wedge}{g})}{\partial_{h_{\hat{g}}}}=\frac{\underset{k \in \Omega}{\vee}\left[\beta_{h_{\aleph}}(\stackrel{\wedge}{g}, k) \vee \beta_{h_{b}}(k)\right]}{\underset{k \in \Omega}{\vee}\left[\partial_{h_{\aleph}}(\stackrel{\wedge}{g}, c) \vee \partial_{h_{b}}(k)\right]}, \\
& \psi_{h_{\bar{\aleph}(b)}}(\stackrel{\wedge}{g})=\frac{\bigwedge_{k \in \Omega}\left[\psi_{h_{\aleph}}(\stackrel{\wedge}{g}, k) \wedge \psi_{h_{b}}(k)\right]}{\wedge{ }_{k \in \Omega}\left[\partial_{h_{\aleph}}(\stackrel{\wedge}{g}, c) \wedge \partial_{h_{b}}(k)\right]},
\end{aligned}
$$

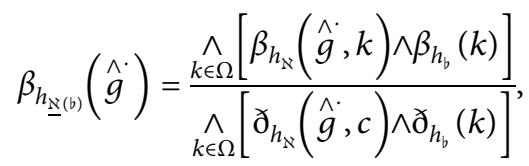

$$
\begin{aligned}
& \psi_{h_{\underline{\aleph}(b)}}(\stackrel{\wedge}{g})=\frac{\underset{k \in \Omega}{\vee}\left[\psi_{h_{\aleph}}(\stackrel{\wedge}{g}, k) \vee \psi_{h_{b}}(k)\right]}{\vee_{k \in \Omega}\left[\partial_{h_{\aleph}}(\stackrel{\wedge}{g}, c) \vee \partial_{h_{b}}(k)\right]},
\end{aligned}
$$

such that

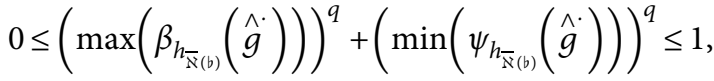

$$
\begin{aligned}
& 0 \leq\left(\min \left(\beta_{h_{\underline{\mathrm{N}}(b)}}(\stackrel{\wedge}{g})\right)^{q}+\left(\max \left(\psi_{h_{\underline{\underline{\mathrm{N}}(b)}}}(\stackrel{\wedge}{g})\right)\right)^{q} \leq 1 .\right.
\end{aligned}
$$

$$
\text { As } \quad(\bar{\aleph}(b), \underline{\aleph}(b)) \quad \text { are } q \text {-ROPHFSs, }
$$
$\bar{\aleph}(b), \aleph(b): q-\operatorname{ROPHFS}(\Omega) \longrightarrow q-\operatorname{RFS}(\Omega)$ are upper and lower approximation operators. The pair

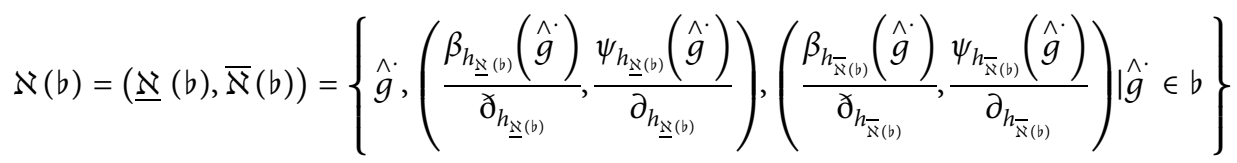

will be called $q$-rung orthopair HFRS. For simplicity,

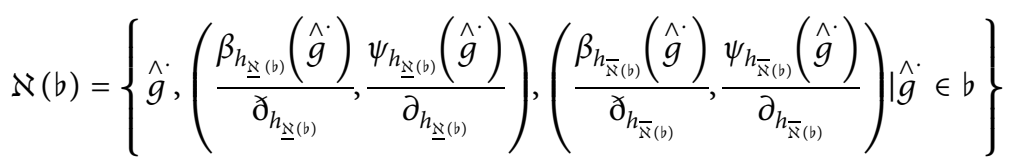

is represented as $\aleph(b)=((\underline{\xi} / \underline{\partial}, \underline{\eta} / \partial),(\bar{\beta} / \bar{\partial}, \bar{\psi} / \bar{\partial}))$ and known as $q$-ROPHFRV.

Definition 21. Let $\aleph\left(b_{1}\right)=\left(\underline{\aleph}\left(b_{1}\right), \bar{\aleph}\left(b_{1}\right)\right) \quad$ and $\aleph\left(b_{2}\right)=\left(\underline{\aleph}\left(b_{2}\right), \bar{\aleph}\left(b_{2}\right)\right)$ be two $q$-ROPHFRSs. Then,

(1) $\aleph\left(b_{1}\right) \cup \aleph\left(b_{2}\right)=\left\{\left(\underline{\aleph}\left(b_{1}\right) \cup \underline{\aleph}\left(b_{2}\right)\right)\right.$, $\left.\left(\bar{\aleph}\left(b_{1}\right) \cup \bar{\aleph}\left(b_{2}\right)\right)\right\}$

(2) $\aleph\left(b_{1}\right) \cap \aleph\left(b_{2}\right)=\left\{\left(\underline{\aleph}\left(b_{1}\right) \cap \underline{\aleph}\left(b_{2}\right)\right)\right.$, $\left.\left(\bar{\aleph}\left(b_{1}\right) \cap \bar{\aleph}\left(b_{2}\right)\right)\right\}$

(3) $\aleph\left(b_{1}\right) \oplus \aleph\left(b_{2}\right)=\left\{\left(\underline{\aleph}\left(b_{1}\right) \oplus \underline{\aleph}\left(b_{2}\right)\right),\left(\bar{\aleph}\left(b_{1}\right) \oplus \bar{\aleph}\left(b_{2}\right)\right)\right\}$

(4) $\aleph\left(b_{1}\right) \otimes \aleph\left(b_{2}\right)=\left\{\left(\underline{\aleph}\left(b_{1}\right) \otimes \underline{\aleph}\left(b_{2}\right)\right)\right.$, $\left.\left(\bar{\aleph}\left(b_{1}\right) \otimes \bar{\aleph}\left(b_{2}\right)\right)\right\}$

(5) $\aleph\left(b_{1}\right) \subseteq \aleph\left(b_{2}\right)=\left\{\left(\underline{\aleph}\left(b_{1}\right) \subseteq \underline{\aleph}\left(b_{2}\right)\right)\right.$ and $\left.\left(\bar{\aleph}\left(b_{1}\right) \subseteq \bar{\aleph}\left(b_{2}\right)\right)\right\}$
(6) $\gamma \aleph\left(b_{1}\right)=\left(\gamma \underline{\aleph}\left(b_{1}\right), \gamma \bar{\aleph}\left(b_{1}\right)\right)$ for $\gamma \geq 1$

(7) $\left(\aleph\left(b_{1}\right)\right)^{\gamma}=\left(\left(\underline{\aleph}\left(b_{1}\right)\right)^{\gamma},\left(\bar{\aleph}\left(b_{1}\right)\right)^{\gamma}\right)$ for $\gamma \geq 1$

(8) $\aleph\left(b_{1}\right)^{c}=\left(\underline{\aleph}\left(b_{1}\right)^{c}, \bar{\aleph}\left(b_{1}\right)^{c}\right)$ where $\underline{\aleph}\left(b_{1}\right)^{c}$ and $\bar{\aleph}\left(b_{1}\right)^{c}$ show the complement of $q$-RFR approximation operators $\underline{\aleph}\left(b_{1}\right)$ and $\bar{\aleph}\left(b_{1}\right)$, which is $\underline{\aleph}\left(b_{1}\right)^{c}=\left(\psi_{h_{\aleph(b)}} / \partial, \widehat{\beta_{h_{\aleph(b)}}} / ð\right)$

(9) $\aleph\left(b_{1}\right)=\aleph\left(b_{2}\right)$ iff $\underline{\aleph}\left(b_{1}\right)=\underline{\aleph}\left(b_{2}\right)$ and $\bar{\aleph}\left(b_{1}\right)=\bar{\aleph}\left(b_{2}\right)$

Definition 22. The score function for $q$-ROPHFRV

$$
\aleph(b)=(\underline{\aleph}(b), \bar{\aleph}(b))=\left(\left(\frac{\underline{\bar{g}}}{\underline{\partial}}, \frac{\underline{\eta}}{\partial}\right),\left(\frac{\bar{\beta}}{\bar{\partial}}, \overline{\bar{\psi}}\right)\right)
$$

is given as 


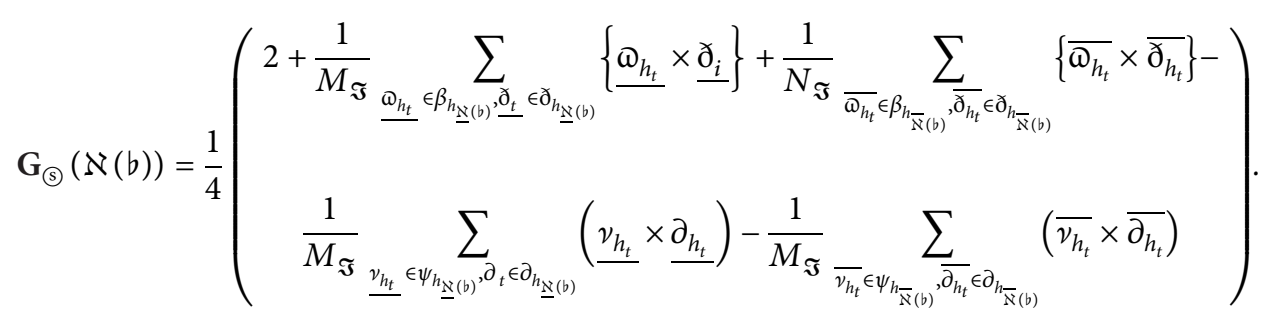

The accuracy function for $q$-ROPHFRV $\aleph(b)=(\underline{\aleph}(b), \bar{\aleph}(b))=((\underline{\xi} / \underline{\partial}, \eta / \partial),(\bar{\beta} / \bar{\partial}, \bar{\psi} / \bar{\partial}))$ is given as

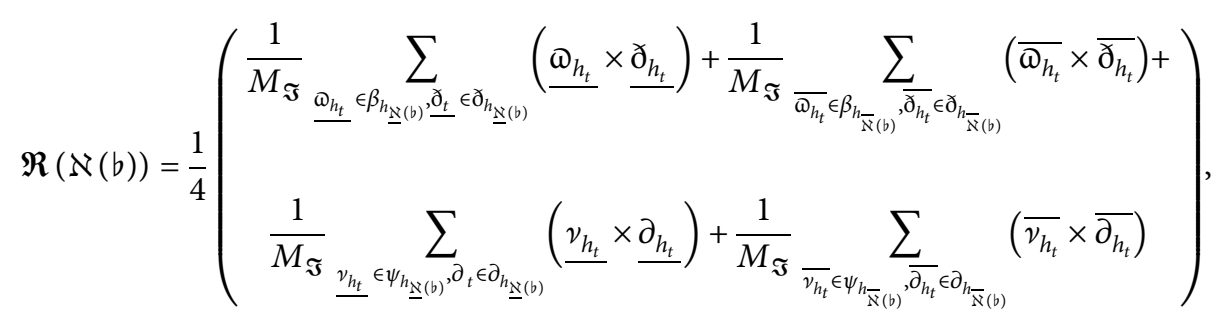

where $M_{\mathfrak{\Im}}$ and $N_{\mathfrak{\Im}}$ represent the number of elements in $\beta_{h_{g}}$ and $\psi_{h_{g}}$, respectively.

Definition 23. Suppose $\aleph\left(b_{1}\right)=\left(\underline{\aleph}\left(b_{1}\right), \bar{\aleph}\left(b_{1}\right)\right)$ and $\aleph\left(b_{2}\right)=\left(\underline{\aleph}\left(b_{2}\right), \bar{\aleph}\left(b_{2}\right)\right)$ are two $q$-ROPHFRVs. Then,

(1) If $\mathbf{G}_{\odot}\left(\aleph\left(b_{1}\right)\right)>\mathbf{G}_{\odot}\left(\aleph\left(b_{2}\right)\right)$, then $\aleph\left(b_{1}\right)>\aleph\left(b_{2}\right)$

(2) If $\mathbf{G}_{\odot}\left(\aleph\left(b_{1}\right)\right)<\mathbf{G}_{\odot}\left(\aleph\left(b_{2}\right)\right)$, then $\aleph\left(b_{1}\right)<\aleph\left(b_{2}\right)$

(3) If $\mathbf{G}_{\circledast}\left(\aleph\left(b_{1}\right)\right)=\mathbf{G}_{\odot}\left(\aleph\left(b_{2}\right)\right)$, then

(a) If $\mathfrak{R}\left(\aleph\left(b_{1}\right)\right)>\mathfrak{R}\left(\aleph\left(b_{2}\right)\right)$, then $\aleph\left(b_{1}\right)>\aleph\left(b_{2}\right)$

(b) If $\Re\left(\aleph\left(b_{1}\right)\right)<\Re\left(\aleph\left(b_{2}\right)\right)$, then $\aleph\left(b_{1}\right)<\aleph\left(b_{2}\right)$

(c) If $\Re\left(\aleph\left(b_{1}\right)\right)=\Re\left(\aleph\left(b_{2}\right)\right)$, then $\aleph\left(b_{1}\right)=\aleph\left(b_{2}\right)$

\section{5. $q$-Rung Orthopair Probabilistic Hesitant Fuzzy Rough Aggregation Operators}

In this section, we propose a new idea of $q$-ROPHF rough AOPs by embedding the notions of the RS and $q$-ROPHF AOPs to get aggregation concepts of $q$-ROPHFREWA and
q-ROPHFREWGA. Furthermore, some fundamental properties of the notion are discussed.

5.1. q-Rung Orthopair Probabilistic Hesitant Fuzzy Rough Einstein Weighted Averaging Operator

Definition 24. Let $\aleph\left(b_{t}\right)=\left(\underline{\aleph}\left(b_{t}\right), \bar{\aleph}\left(b_{t}\right)\right)(t=1,2,3,4$, $\ldots, n)$ be the collection of $q$-ROPHFRVs. Then, the $q$ ROPHFREWA operator is determined by

$$
\begin{aligned}
q- & \operatorname{ROPHFRWA}\left(\aleph\left(b_{1}\right), \aleph\left(b_{2}\right), \ldots, \aleph\left(b_{n}\right)\right) \\
& =\left(\underset{t=1}{n} \gamma_{t} \underline{\aleph}\left(b_{t}\right), \underset{t=1}{n} \gamma_{t} \bar{\aleph}\left(b_{t}\right)\right),
\end{aligned}
$$

where $\gamma=\left(\gamma_{1}, \gamma_{2}, \ldots, \gamma_{n}\right)^{T}$ are the weight vector such that $\oplus_{t=1}^{n} \gamma_{t}=1$ and $0 \leq \gamma_{t} \leq 1$ and $\partial_{h_{t}}$ and $\partial_{h_{t}}$ are probabilistic terms such that $\oplus_{t=1}^{n} \partial_{h_{t}}=1$ and $\oplus_{t=1}^{n} \partial_{h_{t}}=1$.

Theorem 1. Let $\aleph\left(b_{t}\right)=\left(\underline{\mathrm{N}}\left(b_{t}\right), \overline{\mathrm{N}}\left(b_{t}\right)\right)(t=1,2,3,4$, $\ldots, n)$ be the collection of $q$-ROPHFRVs. Then, the $q$-ROPHFREWA operator is defined by 


$$
\begin{aligned}
& q \text { - ROPHFREWA }\left(\aleph\left(b_{1}\right), \aleph\left(b_{2}\right), \ldots, \aleph\left(b_{n}\right)\right) \\
& =\left(\stackrel{\oplus}{t=1}_{t}^{n} \gamma_{t} \underline{\aleph}\left(b_{t}\right), \underset{t=1}{\stackrel{n}{\oplus}} \gamma_{t} \bar{\aleph}\left(b_{t}\right)\right) \text {, } \\
& U \varpi_{h_{t}} \in \beta_{\underline{\underline{\mathbb{N}}}_{(b)}}, \partial_{h_{t}} \in \partial_{h_{\underline{\underline{X}}(b)}}\left(\frac{\sqrt[q]{\otimes_{t=1}^{n}\left(1+\Phi_{h_{t}}^{q}\right)^{\gamma t}-\otimes_{t=1}^{n}\left(1-\varpi_{h_{t}}^{q}\right)^{\gamma t}} / \sqrt[q]{\otimes_{t=1}^{n}\left(1+\Phi_{h_{t}}^{q}\right)^{\gamma t}+\otimes_{t=1}^{n}\left(1-\Phi_{h_{t}}^{q}\right)^{\gamma t}}}{\otimes_{t=1}^{n} \partial_{h_{t}}}\right), \\
& U_{v_{h_{t}}} \in \psi_{h_{\underline{\underline{X}}(b)}}, \partial_{h_{t}} \in \partial_{h_{\underline{\underline{X}}(b)}}\left(\frac{\sqrt[q]{2 \otimes_{t=1}^{n}\left(v_{h_{t}}^{q}\right)^{\gamma t}} / \sqrt[q]{\otimes_{t=1}^{n}\left(2-v_{h_{t}}^{q}\right)^{\gamma t}+\otimes_{t=1}^{n}\left(v_{h_{t}}^{q}\right)^{\gamma t}}}{\otimes_{t=1}^{n} \partial_{h_{t}}}\right),
\end{aligned}
$$

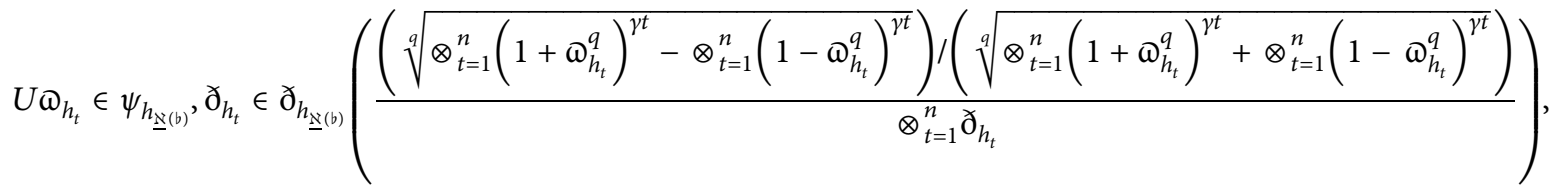

$$
\begin{aligned}
& U_{v_{h_{t}}} \in \psi_{h_{\underline{\underline{X}}(b)}}, \partial_{h_{t}} \in \partial_{h_{\underline{\underline{X}}(b)}}\left(\frac{\sqrt[q]{2 \otimes_{t=1}^{n}\left(v_{h_{t}}^{q}\right)^{\gamma t}} /\left(\sqrt[q]{\otimes_{t=1}^{n}\left(2-v_{h_{t}}^{q}\right)^{\gamma t}+\otimes_{t=1}^{n}\left(v_{h_{t}}^{q}\right)^{\gamma t}}\right)}{\otimes_{t=1}^{n} \partial_{h_{t}}}\right),
\end{aligned}
$$

where $\gamma=\left(\gamma_{1}, \gamma_{2}, \ldots, \gamma_{n}\right)^{T}$ are the weight vector such that $\oplus_{t=1}^{n} \gamma_{t}=1$ and $0 \leq \gamma_{t} \leq 1$ and $\partial_{h_{t}}$ and $\partial_{h_{t}}$ are probabilistic terms such that $\oplus_{t=1}^{n} \partial_{h_{t}}=1$ and $\oplus_{t=1}^{n} \partial_{h_{t}}=1$.
Proof. We will prove the aforesaid theorem by utilizing mathematical induction. Suppose $n=2$. Then,

$$
\begin{aligned}
& \left(\aleph\left(b_{1}\right) \otimes \aleph\left(b_{2}\right)\right) \\
& =\left(\underline{\aleph}\left(b_{1}\right) \otimes \underline{\aleph}\left(b_{2}\right), \bar{\aleph}\left(b_{1}\right) \otimes \bar{\aleph}\left(b_{2}\right)\right), \\
& q-\operatorname{ROPHFREWG}\left(\aleph\left(b_{1}\right), \aleph\left(b_{2}\right)\right) \\
& =\left(\stackrel{\otimes}{t=1}_{t}^{2} \gamma_{t} \underline{\aleph}\left(b_{t}\right), \stackrel{\otimes}{t=1}_{t}^{2} \gamma_{t} \bar{\aleph}\left(b_{t}\right)\right)
\end{aligned}
$$

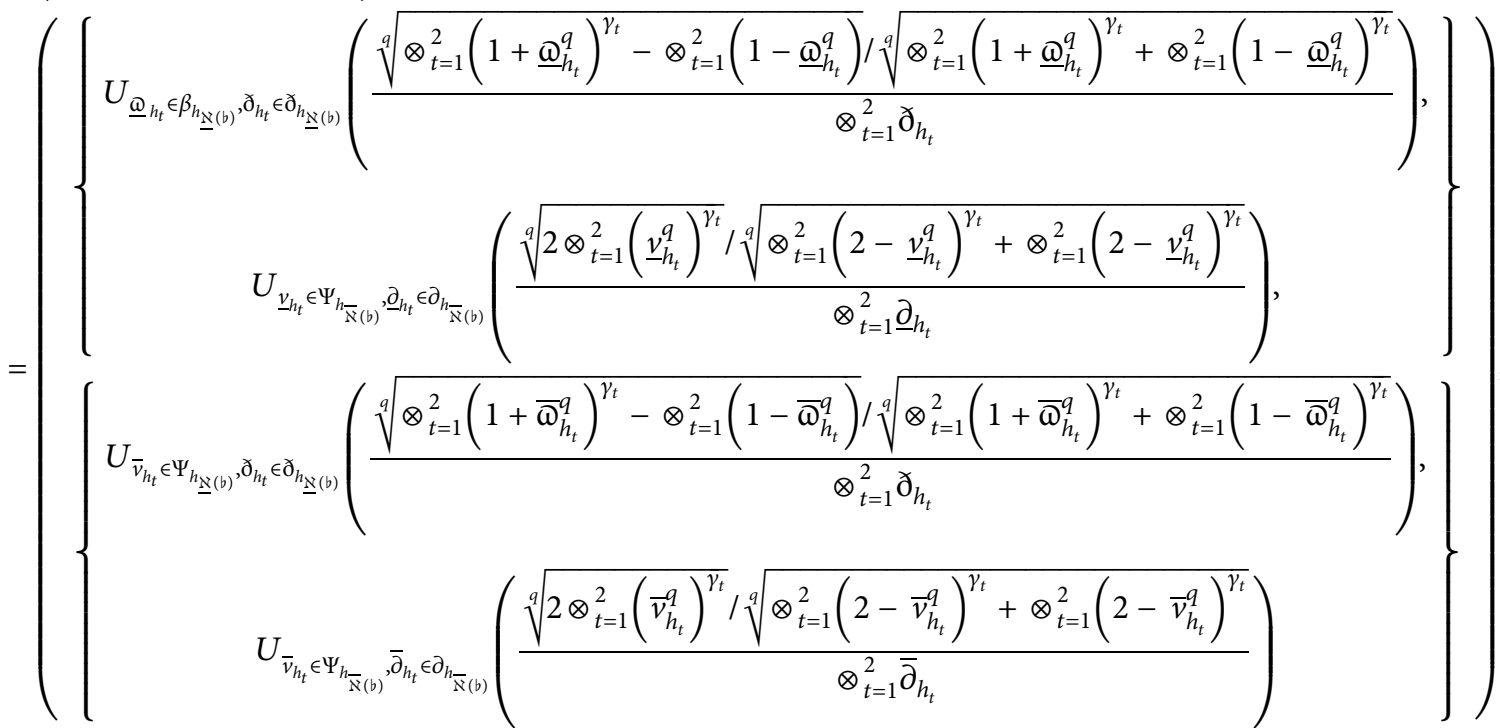

and the result is true for $n=2$. Now, suppose the result is true for $n=k$. 
$q-\operatorname{ROPHFREWA}\left(\aleph\left(b_{1}\right), \aleph\left(b_{2}\right) \ldots, \aleph\left(b_{k}\right)\right)$

$=\left(\underset{t=1}{\stackrel{\oplus}{\oplus}} \gamma_{t} \underline{\aleph}\left(b_{t}\right), \underset{t=1}{\stackrel{\oplus}{\oplus}} \gamma_{t} \bar{\aleph}\left(b_{t}\right)\right)$

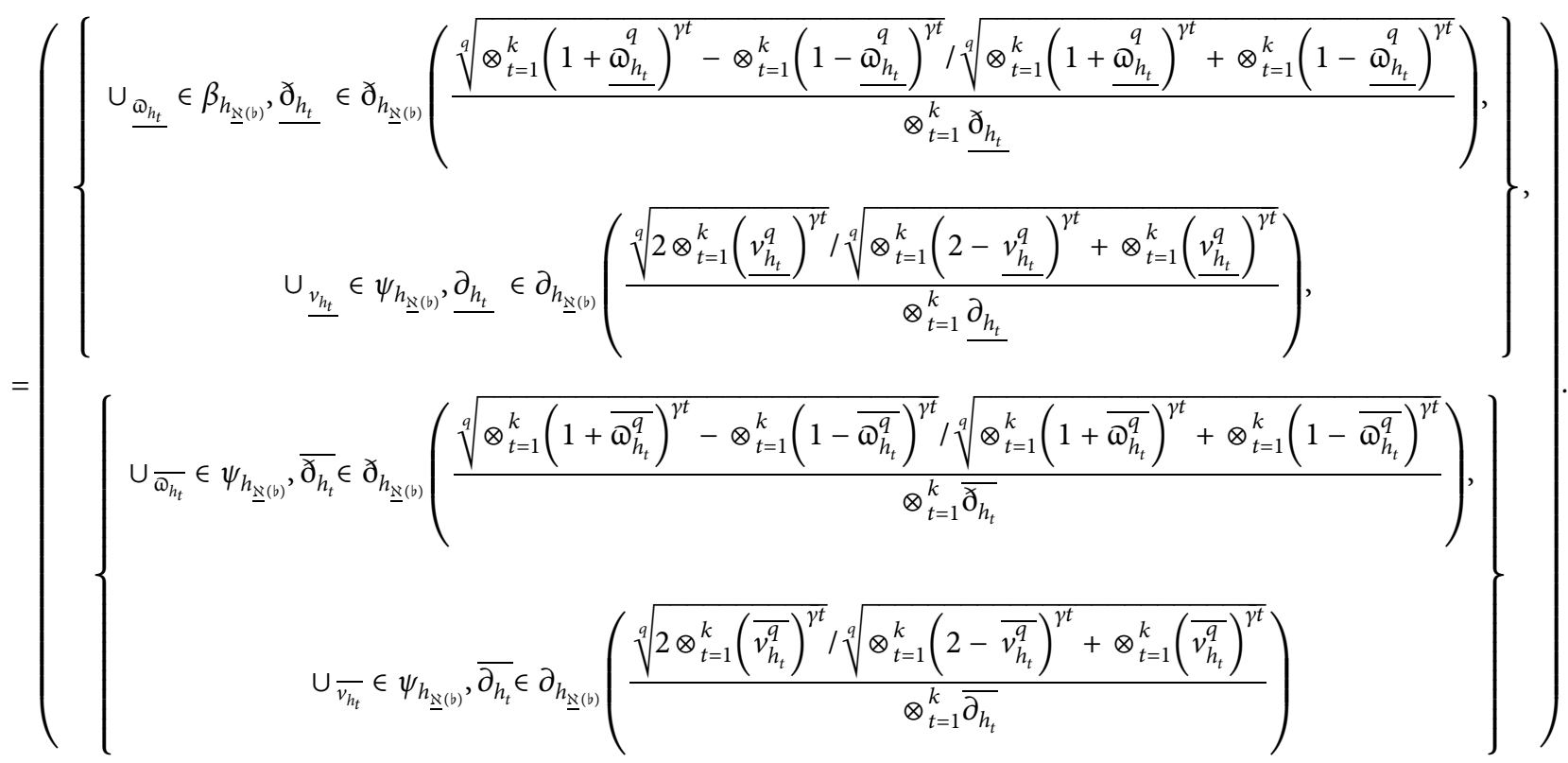

Next, we shall show that the result is true for $n=k+1$.

$q-\operatorname{ROPHFREWA}\left(\aleph\left(b_{1}\right), \aleph\left(b_{2}\right) \ldots \aleph\left(b_{k}\right), \aleph\left(b_{k+1}\right)\right)$

$=\left(\underset{t=1}{\stackrel{\oplus}{\oplus}} \gamma_{t} \underline{\aleph}\left(b_{t}\right) \oplus\left(\underline{\aleph}\left(b_{k+1}\right)\right)^{w_{k+1}}, \underset{t=1}{\stackrel{\oplus}{\oplus}} \gamma_{t} \bar{\aleph}\left(b_{t}\right) \oplus\left(\bar{\aleph}\left(b_{k+1}\right)\right)^{w_{k+1}}\right)$

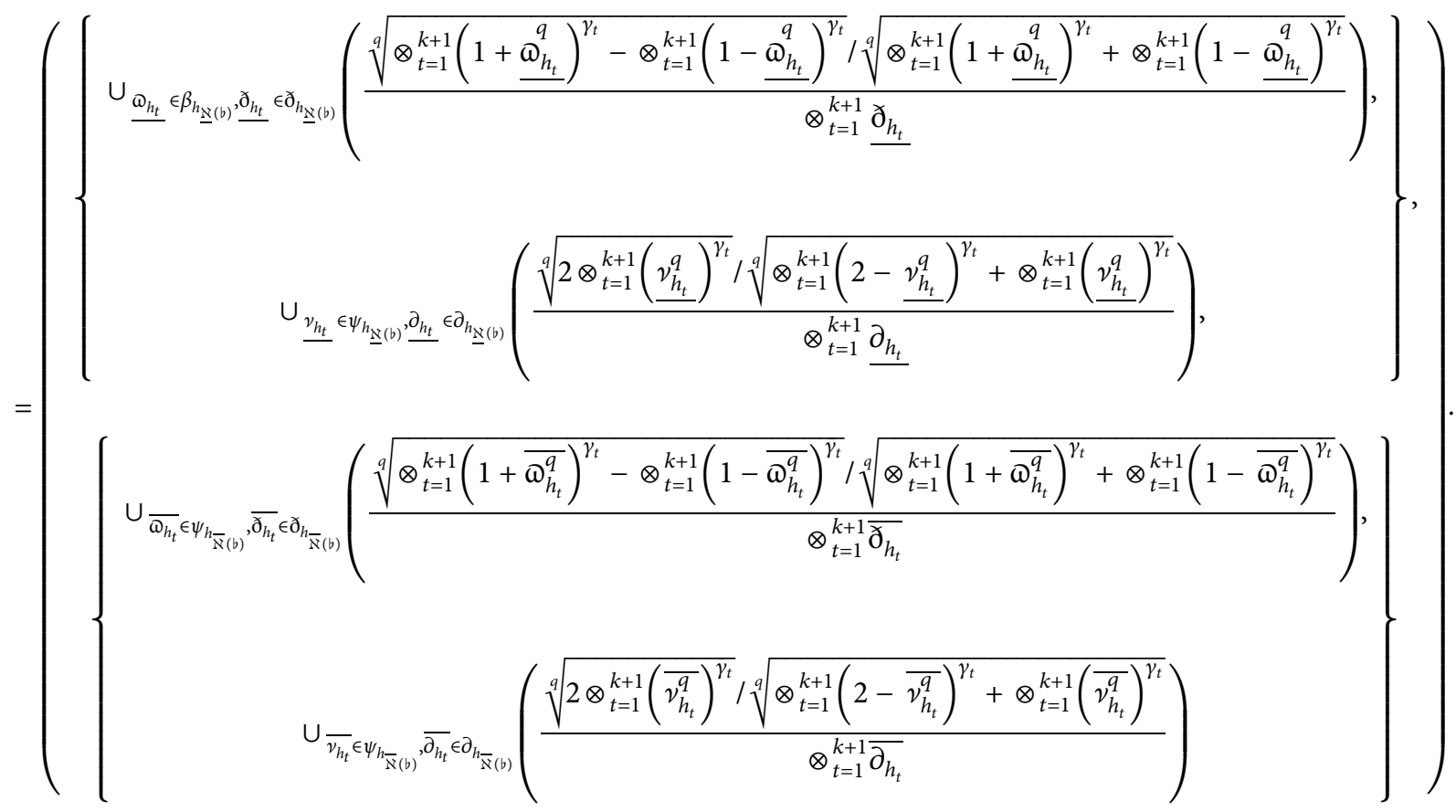


Hence, the result is true for $n=k+1$. Therefore, the result is true for all $n \geq 1$.

Theorem 2. Let $\aleph\left(b_{t}\right)=\left(\underline{\aleph}\left(b_{t}\right), \bar{\aleph}\left(b_{t}\right)\right)(t=1,2,3, \ldots, n)$ be the collection of $q$-ROPHFRVs, $\gamma=\left(\gamma_{1}, \gamma_{2}, \ldots, \gamma_{n}\right)^{T}$ be the weight vector such that $\left.\gamma_{t} \in 0,1\right]$ and $\oplus_{t=1}^{n} \gamma_{t}=1$, and $\partial_{h_{t}}$ and $\partial_{h_{t}}$ be probabilistic terms such that $\oplus_{t=1}^{n} \partial_{h_{t}}=1$ and $\oplus_{t=1}^{n} \partial_{h_{t}}=$ 1. Then, the q-ROPHFRWA operator satisfies the following properties:

(1) Idempotency: if $\aleph\left(b_{t}\right)=\mathrm{F}(b)$ for $t=1,2,3, \ldots, n$ where

$$
\mathrm{F}(b)=(\underline{\varrho}(b), \overline{\mathrm{F}}(b))=\left(\left(\frac{b_{h(x)}}{\overline{\partial_{h(x)}}}, \frac{d_{h(x)}}{\overline{\partial_{h(x)}}}\right),\left(\frac{\bar{b}_{h(x)}}{\overline{\partial_{h(x)}}}, \frac{\overline{d_{h(x)}}}{\overline{\partial_{h(x)}}}\right)\right),
$$

then

$$
q-\operatorname{ROPHFRWG}\left(\aleph\left(b_{1}\right), \aleph\left(b_{2}\right), \ldots, \aleph\left(b_{n}\right)\right)=\mathrm{F}(b) .
$$

(2) Boundedness: let $(\aleph(b))_{\min }=\left(\min _{t} \aleph\left(b_{t}\right)\right.$, $\left.\max _{t} \bar{\aleph}\left(b_{t}\right)\right)$ and $(\aleph(b))_{\max }=\left(\max _{t} \underline{\underline{\aleph}}\left(b_{t}\right)\right.$, $\left.\min _{t} \bar{\aleph}\left(b_{t}\right)\right)$. Then,

$(\aleph(b))_{\min } \leq q-\operatorname{ROPHFRWG}\left(\aleph\left(b_{1}\right), \aleph\left(b_{2}\right), \ldots, \aleph\left(b_{n}\right)\right)$

$$
\leq(\aleph(b))_{\max } \text {. }
$$

(3) Monotonicity: suppose $\quad \mathrm{F}(b)=\left(\underline{\varrho}\left(b_{t}\right), \overline{\mathrm{F}}\left(b_{t}\right)\right)$ $(t=1,2, \ldots, n)$ is another collection of $q$-ROPHFRVs such that $\underline{\underline{\mathrm{g}}}\left(b_{t}\right) \leq \underline{\aleph}\left(b_{t}\right)$ and $\overline{\mathrm{F}}\left(b_{t}\right) \leq \bar{\aleph}\left(b_{t}\right)$. Then,

$$
\begin{aligned}
q-\operatorname{ROPHFRWG}\left(\mathrm{F}\left(b_{1}\right), \mathrm{F}\left(b_{2}\right), \ldots, \mathrm{F}\left(b_{n}\right)\right) \\
\quad \leq q-\operatorname{ROPHFRWG}\left(\aleph\left(b_{1}\right), \aleph\left(b_{2}\right), \ldots, \aleph\left(b_{n}\right)\right) .
\end{aligned}
$$

Proof.

(1) Idempotency: as $\aleph\left(b_{t}\right)=\mathrm{F}(b) \quad$ (for all $t=1,2,3, \ldots, n) \quad$ where $\quad \mathrm{F}\left(b_{t}\right)=(\underline{\mathrm{F}}(b), \overline{\mathrm{F}}(b))=$ $\left(\underline{\left(b_{h(x)}\right.} / \underline{\partial_{h(x)}}, \underline{d_{h(x)}} / \underline{\left.\partial_{h(x)}\right)},\left(\overline{b_{h(x)}} / \overline{\partial_{h(x)}}, \overline{d_{h(x)}} /\right.\right.$ $\left.\overline{\left.\partial_{h(x)}\right)}\right)$, it follows that

$q-\operatorname{ROPHFREWA}\left(\aleph\left(b_{1}\right), \aleph\left(b_{2}\right), \ldots, \aleph\left(b_{n}\right)\right)$

$$
\begin{aligned}
& =\left(\stackrel{\oplus}{t=1}_{t}^{n} \underline{\aleph}\left(b_{t}\right), \underset{t=1}{n} \gamma_{t} \bar{\aleph}\left(b_{t}\right)\right)
\end{aligned}
$$

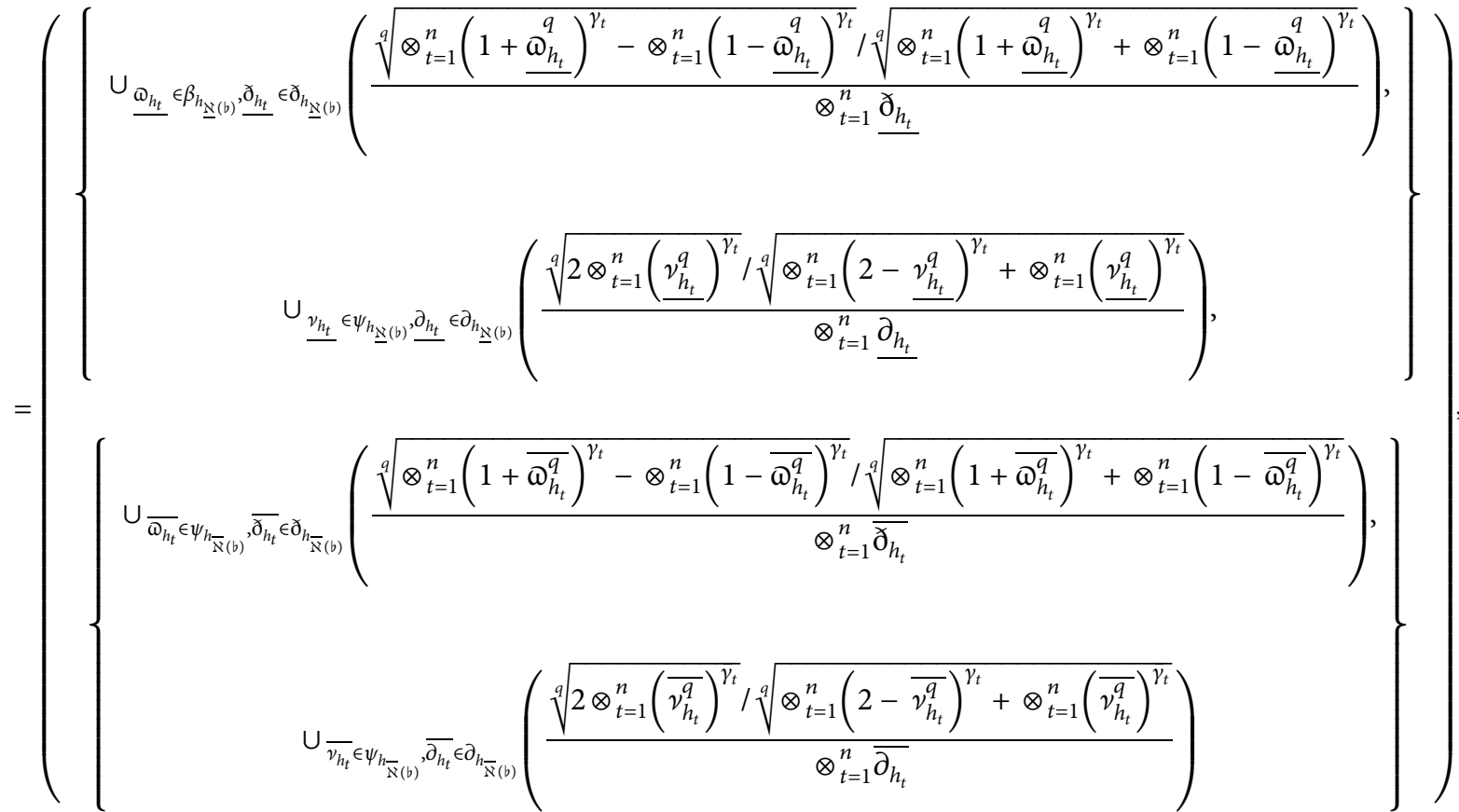

for all $\quad t, \quad(\underline{\mathrm{F}}(b), \overline{\mathrm{F}}(b))=\left(\left(b_{h(x)} / \partial_{h(x)}\right.\right.$, $\left.d_{(h)} / \partial_{h(x)}\right),\left(\bar{b}_{h(x)} / \overline{\partial_{h(x)}}, \overline{\left.d_{h(x)} / \bar{\partial}_{h(x)}\right)}\right)$. It follows that 


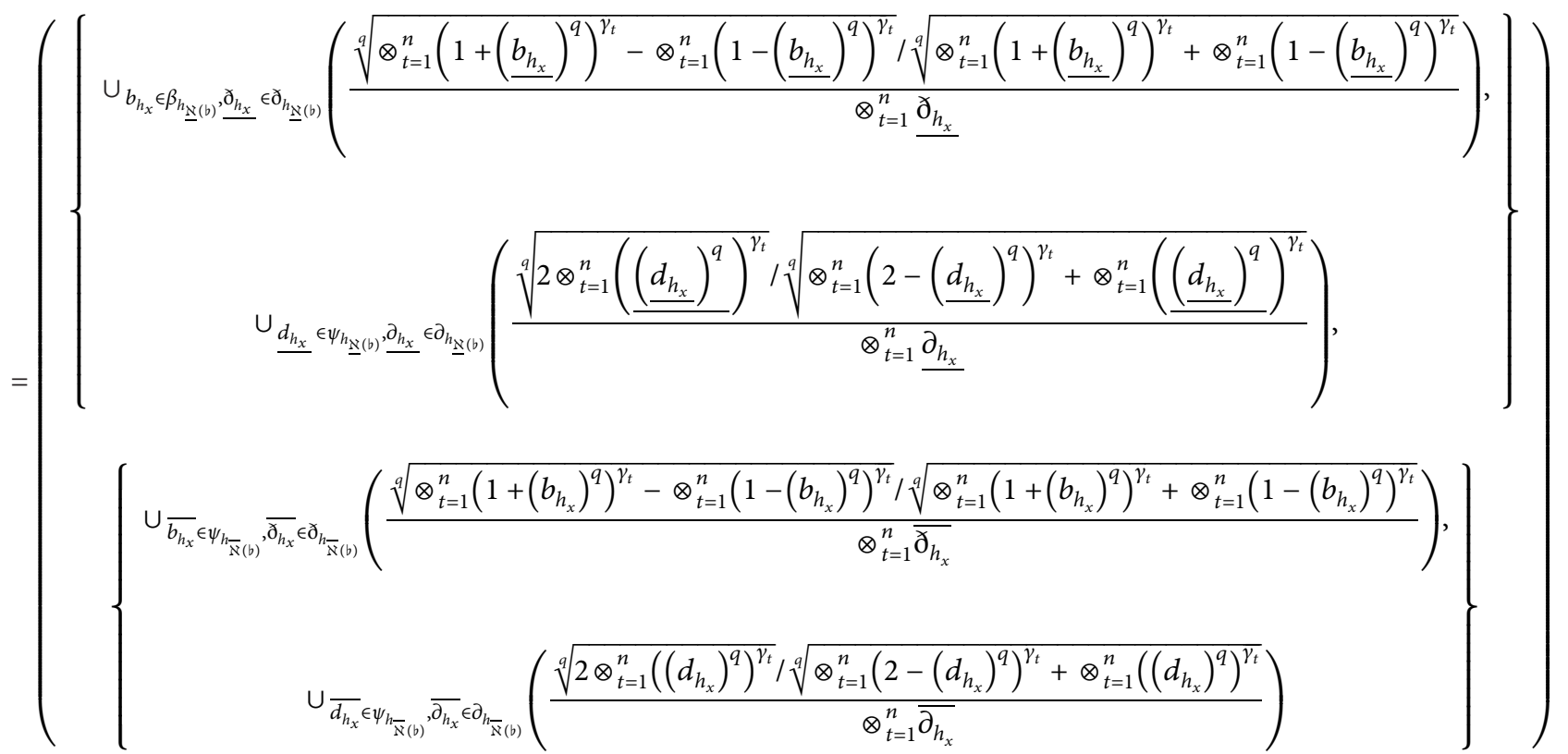

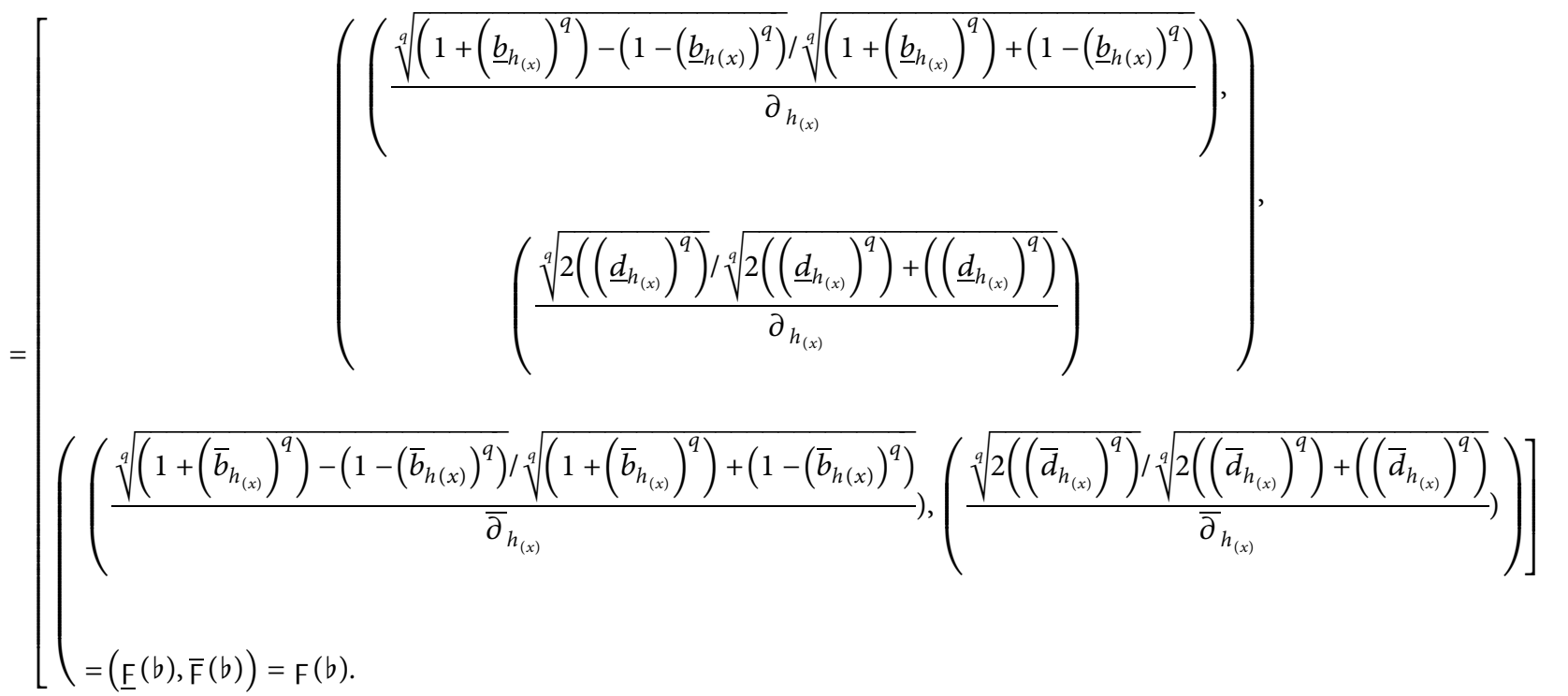

(44)

Hence, $\quad q$-ROPHFREWG $\quad\left(\aleph\left(b_{1}\right), \aleph\left(b_{2}\right), \ldots\right.$, $\left.\aleph\left(b_{n}\right)\right)=\mathrm{F}(b)$.

(2) Boundedness: 


$$
\begin{aligned}
& (\underline{\aleph}(b))^{-}=\left[\begin{array}{l}
\left.\left(\min _{t}\left\{\frac{\Phi_{h_{t}}}{\overline{\partial_{h_{t}}}}\right\}, \max _{t}\left\{\frac{v_{h_{t}}}{\overline{\partial_{h_{t}}}}\right\}\right)\right], \\
\left(\min _{t}\left\{\frac{\overline{\Phi_{h_{t}}}}{\overline{\partial_{h_{t}}}}\right\}, \max _{t}\left\{\frac{\overline{\nu_{h_{i}}}}{\overline{\partial_{h_{t}}}}\right\}\right)
\end{array}\right] \\
& (\underline{\underline{ }}(b))^{+}=\left[\begin{array}{l}
\left.\left(\max _{t}\left\{\frac{\Phi_{h_{t}}}{\overline{\partial_{h_{t}}}}\right\}, \min _{t}\left\{\frac{\bar{\nu}_{h_{t}}}{\overline{\partial_{h_{t}}}}\right\}\right)\right], \\
\left.\left(\max _{t}\left\{\frac{\overline{\Phi_{h_{t}}}}{\overline{\partial_{h_{t}}}}\right\}, \min _{t}\left\{\frac{\overline{\nu_{h_{i}}}}{\overline{\partial_{h_{t}}}}\right\}\right)\right]
\end{array}\right.
\end{aligned}
$$

and $\aleph\left(b_{t}\right)=\left[\left(\xi / \underline{\partial}_{t}, \eta / \partial_{t}\right),\left(\overline{\beta_{t}} / \overline{\partial_{h_{t}}}, \bar{\psi}_{t} / \overline{\partial_{h_{t}}}\right)\right]$ to prove that

$$
\begin{aligned}
(\aleph(b))^{-} & \leq q-\operatorname{ROPHFREWG}\left(\aleph\left(b_{1}\right), \aleph\left(b_{2}\right), \ldots, \aleph\left(b_{n}\right)\right) \\
& \leq(\aleph(b))^{+} .
\end{aligned}
$$

Let $f(y)=\sqrt[3]{1-y^{3} / 1+y^{3}}, y \in 0,1$.] Then, $f^{\prime}(y)=$ $-2 y /\left(1+y^{3}\right)^{3} \sqrt[3]{\left(1-y^{3} / 1+y^{3}\right)^{-2}}<0$. Thus, $f(y)$ is a decreasing function over $[0,1]$. Since $\left\{\underline{\varpi_{h_{\max }}}\right\} \leq\left\{\underline{\varpi_{h_{t}}}\right\} \leq\left\{\underline{\omega_{h_{\min }}}\right\} \quad$ for all $i$,

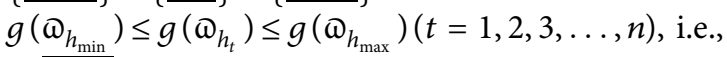

$$
\Leftrightarrow \sqrt[3]{\frac{1-\left(\underline{\omega_{h_{\min }}}\right)^{3}}{1+\left(\underline{\omega_{h_{\min }}}\right)^{3}}} \leq \sqrt[3]{\frac{1-\left(\underline{\omega_{h_{t}}}\right)^{3}}{1+\left(\underline{\omega_{h_{t}}}\right)^{3}}}
$$$$
\leq \sqrt[3]{\frac{1-\left(\underline{\left.\omega_{h_{\max }}\right)^{3}}\right.}{1+\left(\underline{\left.\omega_{h_{\max }}\right)^{3}}\right.}}, t=1,2,3, \ldots, n
$$

and let $\gamma=\left(\gamma_{1}, \gamma_{2}, \ldots, \gamma_{n}\right)^{T}$ be the weight vector such that $\left.\gamma_{t} \in 0,1\right]$ and $\oplus_{t=1}^{n} \gamma_{t}=1$ and $\partial_{h_{t}}$ be the probabilistic term such that $\oplus_{t=1}^{n} \partial_{h_{t}}=1$; we have

$$
\Leftrightarrow \frac{\sqrt[3]{\otimes_{t=1}^{n}\left(\left(1-\left(\underline{\omega_{h_{\text {min }}}}\right)^{3}\right) /\left(1+\left(\underline{\omega_{h_{\min }}}\right)^{3}\right)\right)^{\gamma_{t}}}}{\otimes_{t=1}^{n} \partial_{h_{t}}} \leq \frac{\sqrt[3]{\otimes_{t=1}^{n}\left(\left(1-\left(\underline{\omega_{h_{t}}}\right)^{3}\right) /\left(1+\left(\underline{\omega_{h_{t}}}\right)^{3}\right)\right)^{\gamma_{t}}}}{\otimes_{t=1}^{n} \partial_{h_{t}}}
$$$$
\leq \frac{\sqrt[3]{\otimes_{t=1}^{n}\left(\left(1-\left(\underline{\Phi_{h_{\max }}}\right)^{3}\right) /\left(1+\left(\underline{\omega_{h_{\max }}}\right)^{3}\right)\right)^{\gamma_{t}}}}{\otimes_{t=1}^{n} \underline{\partial_{h_{t}}}}
$$$$
\Leftrightarrow \frac{\sqrt[3]{\left(\left(1-\left(\underline{\varpi_{h_{\text {min }}}}\right)^{3}\right) /\left(1+\left(\underline{\omega_{h_{\text {min }}}}\right)^{3}\right)\right)^{\oplus_{t=1}^{n} \gamma_{t}}}}{\otimes_{t=1}^{n} \underline{\partial_{h_{t}}}} \leq \frac{\sqrt[3]{\otimes_{t=1}^{n}\left(\left(1-\left(\underline{\varpi_{h_{t}}}\right)^{3}\right) /\left(1+\left(\underline{\omega_{h_{t}}}\right)^{3}\right)\right)^{\gamma_{t}}}}{\otimes_{t=1}^{n} \underline{\partial_{h_{t}}}}
$$$$
\leq \frac{\sqrt[3]{\left(\left(1-\left(\underline{\varpi_{h_{\max }}}\right)^{3}\right) /\left(1+\left(\underline{\varpi_{h_{\max }}}\right)^{3}\right)\right)^{\oplus_{t=1}^{n} \gamma_{t}}}}{\otimes_{t=1}^{n} \partial_{h_{t}}}
$$ 
16

Complexity

$$
\begin{aligned}
& \Leftrightarrow \frac{\Phi_{h_{\max }}}{\otimes_{t=1}^{n} \partial_{h_{t}}} \leq \frac{\sqrt[3]{\otimes_{t=1}^{n}\left(\left(1-\left(\underline{\omega_{h_{t}}}\right)^{3}\right) /\left(1+\left(\underline{\Phi_{h_{t}}}\right)^{3}\right)\right)^{\gamma_{t}}}}{\otimes_{t=1}^{n} \underline{\partial_{h_{t}}}} \\
& \leq \frac{\Phi_{h_{\min }}}{\otimes_{t=1}^{n} \frac{\partial_{h_{t}}}{\partial_{t}}} \\
& \Leftrightarrow \frac{\Phi_{h_{\max }}}{\partial_{h_{\max }}} \leq \frac{\sqrt[3]{\otimes_{t=1}^{n}\left(\left(1-\left(\underline{\Phi_{h_{t}}}\right)^{3}\right) /\left(1+\left(\underline{\Phi_{h_{t}}}\right)^{3}\right)\right)^{\gamma_{t}}}}{\otimes_{t=1}^{n} \underline{\partial_{h_{t}}}}
\end{aligned}
$$

In a similar way, we can show that

$$
\begin{aligned}
\Leftrightarrow \frac{\overline{\bar{\omega}_{h_{\max }}}}{\overline{\partial_{h_{\max }}}} & \leq \frac{\sqrt[3]{\otimes_{t=1}^{n}\left(\left(2-\left(\overline{\bar{\omega}_{h_{t}}}\right)^{3}\right) /\left(\overline{\bar{\omega}_{h_{t}}}\right)^{3}\right)^{\gamma_{t}}}}{\otimes_{t=1}^{n} \overline{\partial_{h_{t}}}} \\
& \leq \frac{\overline{\bar{\omega}_{\min }}}{\overline{\bar{\partial}_{h_{\min }}}}
\end{aligned}
$$

Again, let $g(x)=\sqrt[3]{\left(2-x^{3}\right) / \varkappa^{3}}, x \in(0,1]$; then, $g^{\prime}(x)=\left(-2 / \varkappa^{4}\right) \sqrt[3]{\left(\left(2-\varkappa^{3}\right) / \varkappa^{3}\right)^{-2}}<0$. So, $g(x)$ is a decreasing function on $(0,1]$. Since

$$
\begin{aligned}
& \left\{\underline{v_{h_{\min }}}\right\} \leq\left\{\underline{v_{h_{t}}}\right\} \leq\left\{\underline{v_{h_{\max }}}\right\} \quad \text { for } \quad \text { all } t \text {, } \\
& g\left(\underline{v_{h_{\max }}}\right) \leq \boldsymbol{g}\left(v_{h_{t}}\right) \leq \boldsymbol{g}\left(\underline{v_{h_{\min }}}\right)(t=1,2,3, \ldots, n) \text {, ide., }
\end{aligned}
$$

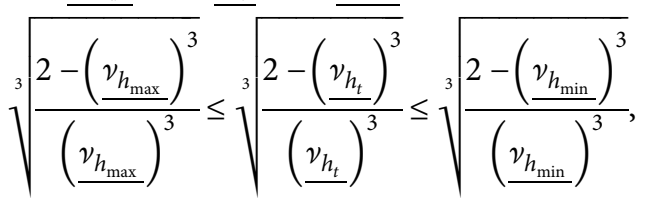

and let $\gamma=\left(\gamma_{1}, \gamma_{2}, \ldots, \gamma_{n}\right)^{T}$ be the weight vector such that $\left.\gamma_{t} \in 0,1\right]$ and $\oplus_{t=1}^{n} \gamma_{t}=1$ and $\partial_{h_{t}}$ be the probabilistic term such that $\oplus_{t=1}^{n} \partial_{h_{t}}=1$; we have

$$
\begin{aligned}
& \Leftrightarrow \frac{\sqrt[3]{\otimes_{t=1}^{n}\left(2-\left(\underline{v_{h_{\max }}}\right)^{3} /\left(\underline{v_{h_{\max }}^{3}}\right)\right)^{\gamma_{t}}}}{\otimes_{t=1}^{n} \underline{\partial_{h_{t}}}} \leq \frac{\sqrt[3]{\otimes_{t=1}^{n}\left(2-\left(\underline{v_{h_{t}}}\right)^{3} /\left(\underline{v_{h_{t}}^{3}}\right)\right)^{\gamma_{t}}}}{\otimes_{t=1}^{n} \underline{\partial_{h_{t}}}} \\
& \leq \frac{\sqrt[3]{\otimes_{t=1}^{n}\left(2-\left(\underline{v_{h_{\max }}}\right)^{3} /\left(\underline{v_{h_{\max }}}\right)^{3}\right)^{\gamma_{t}}}}{\otimes_{t=1}^{n} \partial_{h_{t}}} \\
& \Leftrightarrow \frac{\sqrt[3]{\left(2-\left(\underline{v_{h_{\max }}}\right)^{3} /\left(\underline{v_{h_{\max }}}\right)^{3}\right)^{\oplus_{t=1}^{n} \gamma_{t}}}}{\otimes_{t=1}^{n} \underline{\partial_{h_{t}}}} \leq \frac{\sqrt[3]{\otimes_{t=1}^{n}\left(2-\left(\underline{v_{h_{t}}^{3}}\right) /\left(\underline{v_{h_{t}}}\right)^{3}\right)^{\gamma_{t}}}}{\otimes_{t=1}^{n} \underline{\partial_{h_{t}}}} \\
& \leq \frac{\sqrt[3]{\otimes_{t=1}^{n}\left(2-\left(\underline{v_{h_{\max }}}\right)^{3} /\left(\underline{v_{h_{\max }}}\right)^{3}\right)^{\oplus_{t=1}^{n} \gamma_{t}}}}{\otimes_{t=1}^{n} \partial_{h_{t}}} \\
& \Leftrightarrow \frac{v_{h_{\min }}}{\otimes_{t=1}^{n} \underline{\partial_{h_{t}}}} \leq \frac{\sqrt[3]{\otimes_{t=1}^{n}\left(2-\left(\underline{v_{h_{t}}}\right)^{3} /\left(\underline{v_{h_{t}}}\right)^{3}\right)^{\gamma_{t}}}}{\otimes_{t=1}^{n} \underline{\partial_{h_{t}}}} \leq \frac{\omega_{h_{\max }}}{\otimes_{t=1}^{n} \underline{\partial_{h_{t}}}}, \\
& \Leftrightarrow \frac{v_{h_{\min }}}{\partial_{h_{\min }}} \leq \frac{\sqrt[3]{\otimes_{t=1}^{n}\left(2-\left(\underline{v_{h_{t}}}\right)^{3} /\left(\underline{v_{h_{t}}}\right)^{3}\right)^{\gamma_{t}}}}{\otimes_{t=1}^{n} \partial_{h_{t}}} \leq \frac{v_{h_{\max }}}{\partial_{h_{\min }}} .
\end{aligned}
$$


Similarly, we can show that

$$
\begin{aligned}
& \Leftrightarrow \frac{\overline{v_{h_{\min }}}}{\overline{\partial_{h_{\min }}}} \leq \frac{\sqrt[3]{\sum_{t=1}^{n}\left(2-\left(\overline{v_{h_{t}}}\right)^{3} /\left(\overline{v_{h_{t}}}\right)^{3}\right)^{\gamma_{t}}}}{\sum_{t=1}^{n} \overline{\partial_{h_{t}}}} \\
& \leq \frac{\overline{v_{h_{\max }}}}{\overline{\partial_{h_{\min }}}}
\end{aligned}
$$

Thus, from equations (48), (49), (52), and (53), we have

$$
\begin{aligned}
(\aleph(b))^{-} & \leq q-\operatorname{ROPHFREWG}\left(\aleph\left(b_{1}\right), \aleph\left(b_{2}\right), \ldots, \aleph\left(b_{n}\right)\right) \\
& \leq(\aleph(b))^{+} .
\end{aligned}
$$

(3) Monotonicity: the proof is similar to the proof of (2).

\section{6. $q$-Rung Orthopair Probabilistic Hesitant Fuzzy Rough Einstein Weighted Geometric Aggregation Operator}

In this section, the $q$-ROPHFREWG aggregation operator is introduced, and the key characteristics of the proposed operators are demonstrated.

Definition 25. Let $\aleph\left(b_{t}\right)=\left(\underline{\aleph}\left(b_{t}\right), \bar{\aleph}\left(b_{t}\right)\right)(t=1,2,3,4$, $\ldots, n)$ be the collection of $q$-ROPHFRVs. Then, $q$-ROPHFREWGA operator is determined as

$$
\begin{aligned}
q- & \operatorname{ROPHFREWG}\left(\aleph\left(b_{1}\right), \aleph\left(b_{2}\right), \ldots, \aleph\left(b_{n}\right)\right) \\
& \left.=(\overbrace{t=1}^{n}\left(\underline{\aleph}\left(b_{t}\right)\right)^{\gamma_{t}},{\underset{t=1}{\otimes}}_{t=1}^{n}\left(b_{t}\right))^{\gamma_{t}}\right),
\end{aligned}
$$

where $\gamma=\left(\gamma_{1}, \gamma_{2}, \ldots, \gamma_{n}\right)^{T}$ is the weight vector such that $\oplus_{t=1}^{n} \gamma_{t}=1$ and $0 \leq \gamma_{t} \leq 1$ and $\partial_{h_{t}}$ and $\partial_{h_{t}}$ are probabilistic terms such that $\oplus_{t=1}^{n} \partial_{h_{t}}=1$ and $\oplus_{t=1}^{n} \partial_{h_{t}}=1$.

Theorem 3. Let $\aleph\left(b_{t}\right)=\left(\underline{\aleph}\left(b_{t}\right), \bar{\aleph}\left(b_{t}\right)\right)(t=1,2,3, \ldots, n)$ be the collection of $q$-ROPHFR Vs with the weight vector $\gamma=$ $\left(\gamma_{1}, \gamma_{2}, \ldots, \gamma_{n}\right)^{T}$ such that $\oplus_{t=1}^{n} \gamma_{t}=1$ and $0 \leq \gamma_{t} \leq 1$. Then, the $q$-ROPHFREWG operator is described as

$q-\operatorname{ROPHFREWG}\left(\aleph\left(b_{1}\right), \aleph\left(b_{2}\right), \ldots, \aleph\left(b_{n}\right)\right)$

$$
\begin{aligned}
& =\left(\bigotimes_{t=1}^{n}\left(\underline{\aleph}\left(b_{t}\right)\right)^{\gamma_{t}},{\underset{t=1}{\otimes}}_{t=1}^{n}\left(\bar{\aleph}\left(b_{t}\right)\right)^{\gamma_{t}}\right)
\end{aligned}
$$

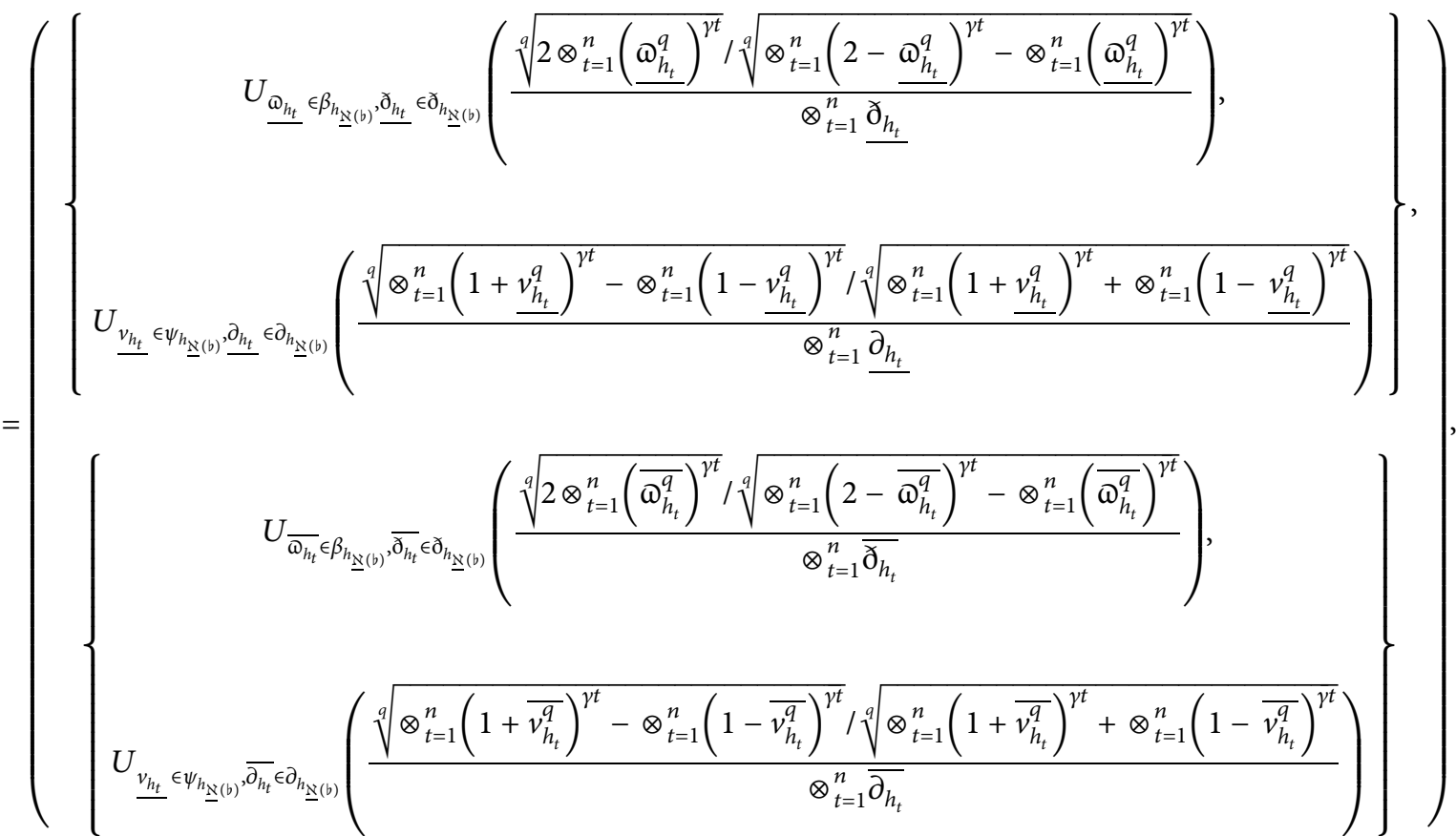


where $\gamma=\left(\gamma_{1}, \gamma_{2}, \ldots, \gamma_{n}\right)^{T}$ is the weight vector such that $\oplus_{t=1}^{n} \gamma_{t}=1$ and $0 \leq \gamma_{t} \leq 1$ and $\partial_{h_{t}}$ and $\partial_{h_{t}}$ are probabilistic terms such that $\oplus_{t=1}^{n} \partial_{h_{t}}=1$ and $\oplus_{t=1}^{n} \partial_{h_{t}}=1$.
Proof. We will prove the aforesaid theorem by utilizing mathematical induction. Suppose $n=2$; then,

$$
\begin{aligned}
& \left(\aleph\left(b_{1}\right) \otimes \aleph\left(b_{2}\right)\right) \\
& =\left(\underline{\aleph}\left(b_{1}\right) \otimes \underline{\aleph}\left(b_{2}\right), \bar{\aleph}\left(b_{1}\right) \otimes \bar{\aleph}\left(b_{2}\right)\right), \\
& q-\operatorname{ROPHFREWG}\left(\aleph\left(b_{1}\right), \aleph\left(b_{2}\right)\right)
\end{aligned}
$$

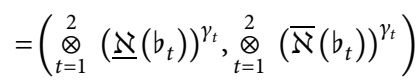

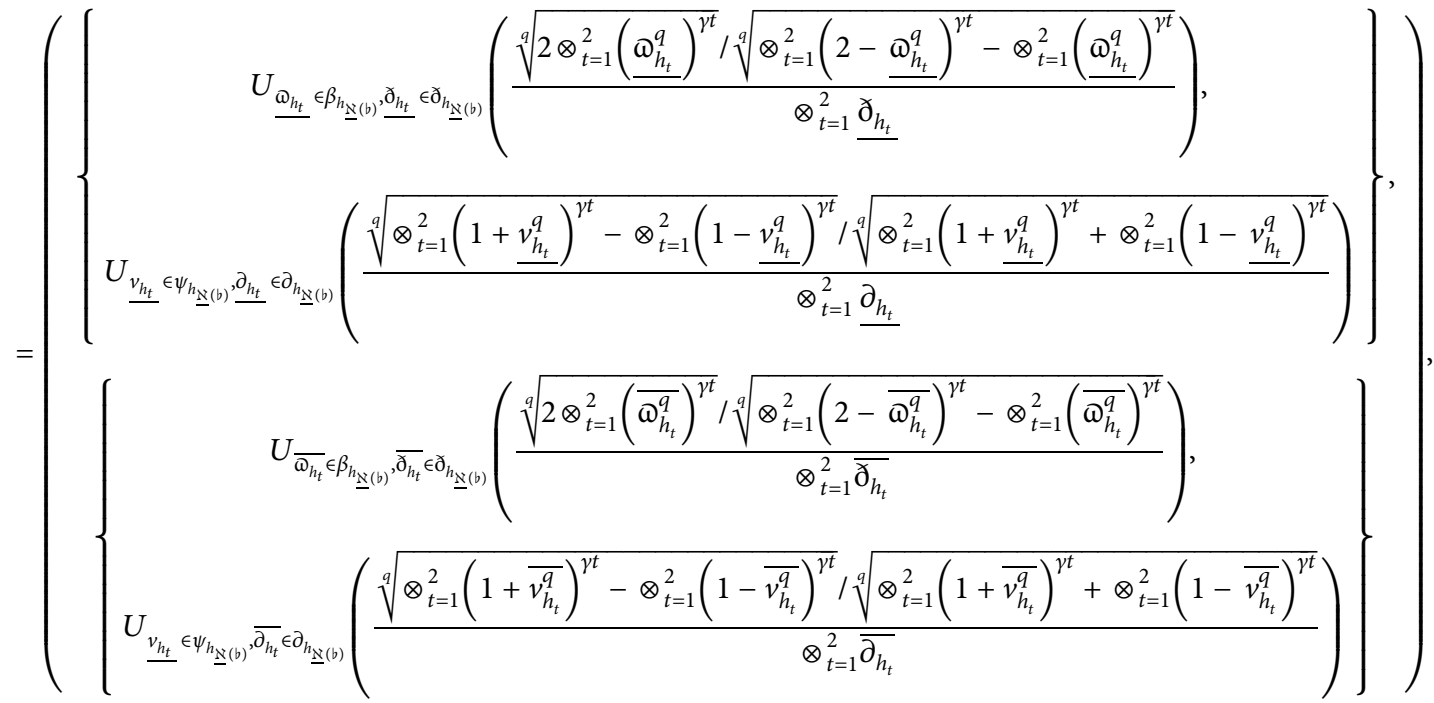

and the result is true for $n=2$. Now, suppose the result is true for $n=k$.

$$
\begin{aligned}
& q-\operatorname{ROPHFREWG}\left(\aleph\left(b_{1}\right), \aleph\left(b_{2}\right) \ldots, \aleph\left(b_{k}\right)\right) \\
& =\left(\underset{t=1}{\otimes}\left(\underline{\aleph}\left(b_{t}\right)\right)^{\gamma_{t}}, \underset{t=1}{\gtrless}\left(\bar{\aleph}\left(b_{t}\right)\right)^{\gamma_{t}}\right)
\end{aligned}
$$

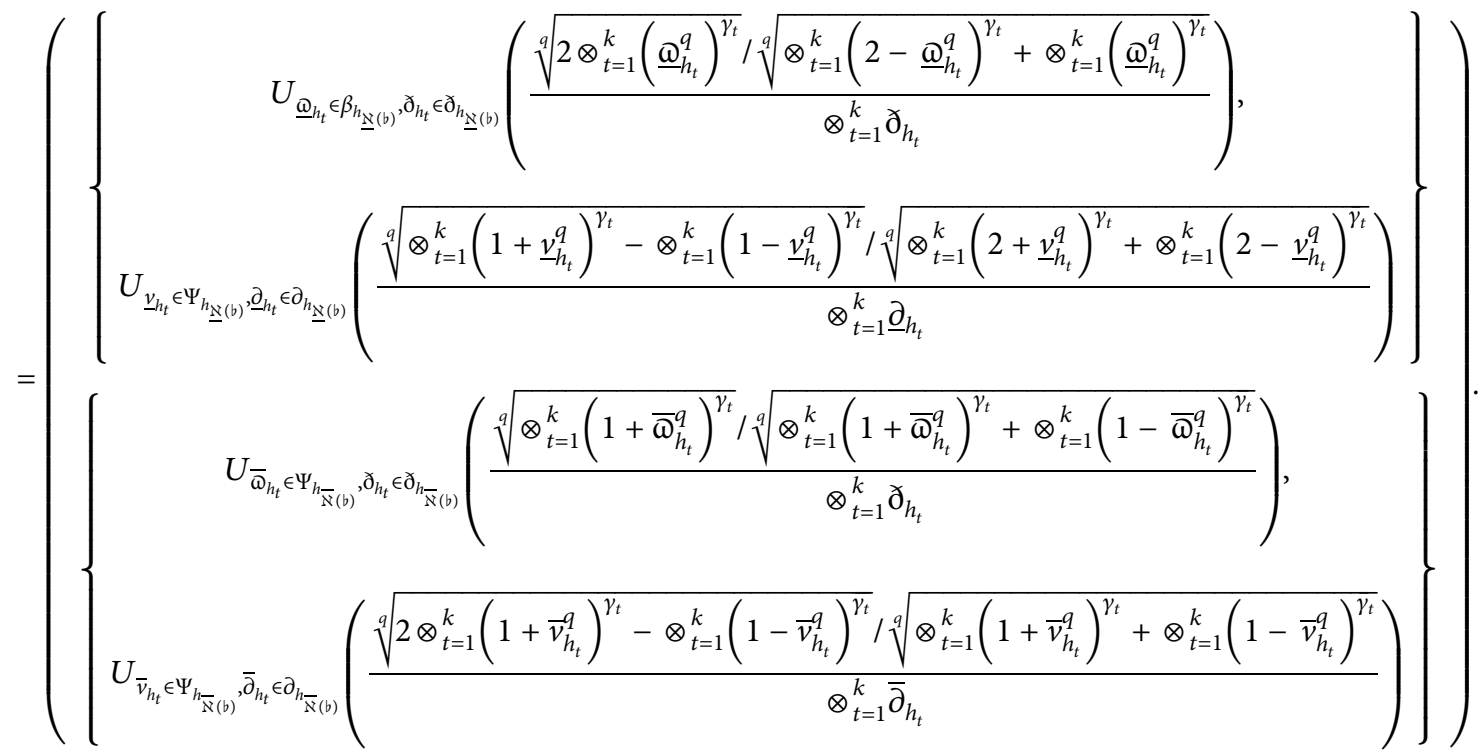


Furthermore, we are going to show that the result holds for $n=k+1$.

$$
\begin{aligned}
& q-\operatorname{ROPHFREWG}\left(\aleph\left(b_{1}\right), \aleph\left(b_{2}\right) \ldots \aleph\left(b_{k}\right), \aleph\left(b_{k+1}\right)\right) \\
& =\left(\stackrel{\otimes}{t=1}_{t}^{k}\left(\left(\underline{\aleph}\left(b_{t}\right)\right)^{\gamma_{t}}\right) \otimes\left(\underline{\aleph}\left(b_{k+1}\right)\right)^{w_{k+1}},{\underset{t=1}{\otimes}}_{t}^{k}\left(\left(\bar{\aleph}\left(b_{t}\right)\right)^{\gamma_{t}}\right) \otimes\left(\bar{\aleph}\left(b_{k+1}\right)\right)^{w_{k+1}}\right)
\end{aligned}
$$

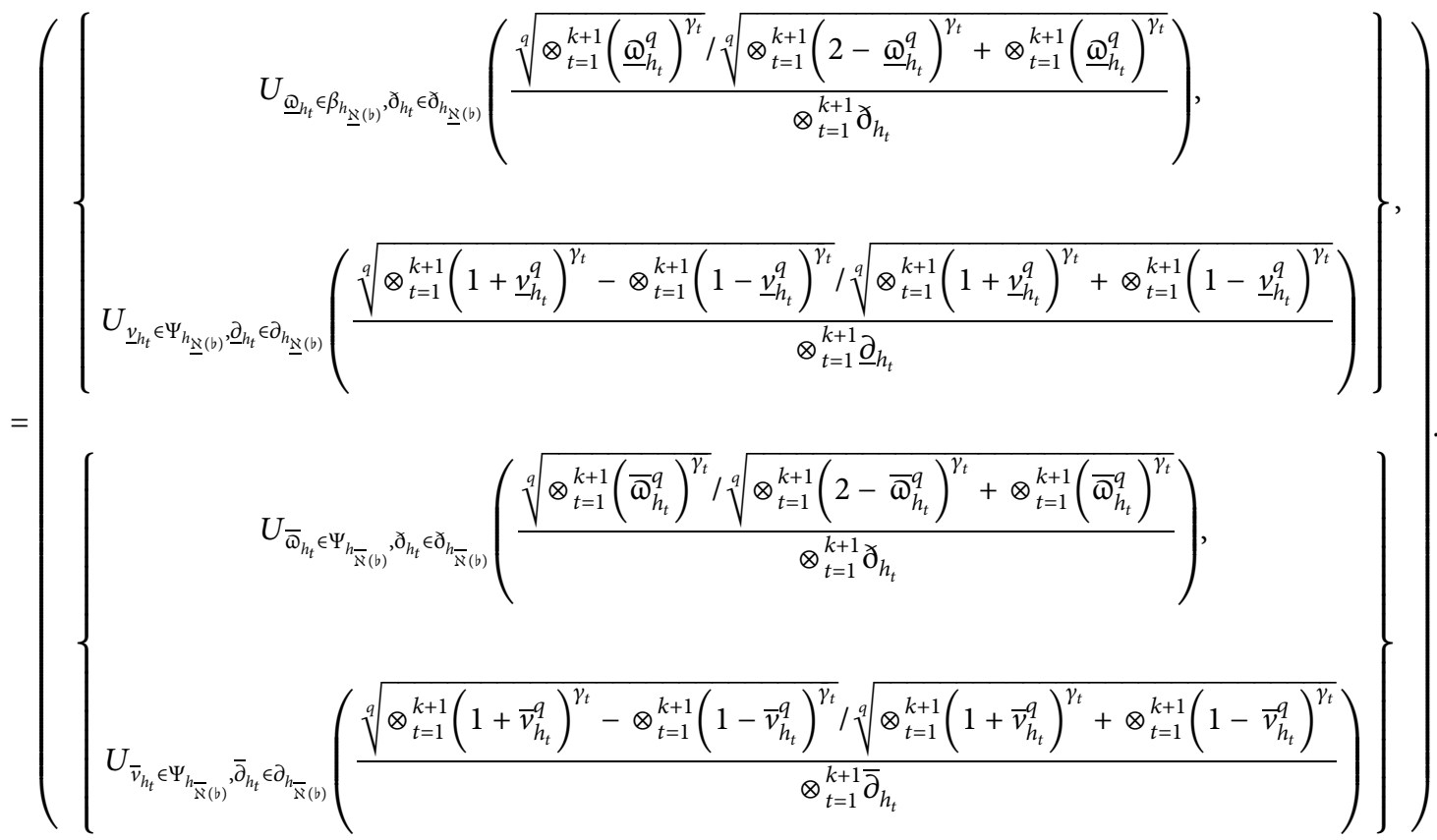

Hence, the result holds for $n=k+1$. Thus, the result is true for all $n \geq 1$.

Theorem 4. Let $\aleph\left(b_{t}\right)=\left(\underline{\aleph}\left(b_{t}\right), \bar{\aleph}\left(b_{t}\right)\right)(t=1,2,3, \ldots, n)$ be the collection of $q$-ROPHFRVs, $\gamma=\left(\gamma_{1}, \gamma_{2}, \ldots, \gamma_{n}\right)^{T}$ be the weight vector such that $\left.\gamma_{t} \in 0,1\right]$ with the property that $\oplus_{t=1}^{n} \gamma_{t}=1$, and $\partial_{h_{t}}$ and $\partial_{h_{t}}$ be probabilistic terms such that $\oplus_{t=1}^{n} \partial_{h_{t}}=1$ and $\oplus_{t=1}^{n} \partial_{h_{t}}=1$. Then, the $q$-ROPHFRWG operator satisfies the following properties:

(1) Idempotency: if $\aleph\left(b_{t}\right)=\mathrm{F}(b)$ for $t=1,2,3, \ldots, n$ where

$$
\mathrm{F}(b)=(\underline{\varrho}(b), \overline{\mathrm{F}}(b))=\left(\left(\underline{b_{h(x)}} / \underline{\partial_{h(x)}}, \underline{d_{h(x)}} / \underline{\partial_{h(x)}}\right),\left(\bar{b}_{h(x)} / \overline{\partial_{h(x)}}, \overline{d_{h(x)}} / \overline{\partial_{h(x)}}\right)\right),
$$

then

$$
q-\operatorname{ROPHFRWG}\left(\aleph\left(b_{1}\right), \aleph\left(b_{2}\right), \ldots, \aleph\left(b_{n}\right)\right)=\mathrm{F}(b)
$$

(2) Boundedness: let $(\aleph(b))_{\min }=\left(\min _{t} \aleph\left(b_{t}\right)\right.$, $\left.\max _{t} \bar{\aleph}\left(b_{t}\right)\right)$ and $(\aleph(b))_{\max }=\left(\max _{t} \underline{\aleph}\left(b_{t}\right)\right.$, $\left.\min _{t} \bar{\aleph}\left(b_{t}\right)\right)$. Then,

$$
\begin{aligned}
(\aleph(b))_{\min } & \leq q-\operatorname{ROPHFRWG}\left(\aleph\left(b_{1}\right), \aleph\left(b_{2}\right), \ldots, \aleph\left(b_{n}\right)\right) \\
& \leq(\aleph(b))_{\max } .
\end{aligned}
$$

(3) Monotonicity: suppose $\quad \mathrm{F}(b)=\left(\underline{\mathrm{F}}\left(b_{t}\right), \overline{\mathrm{F}}\left(b_{t}\right)\right)$ $(t=1,2, \ldots, n)$ is another collection of $q-\bar{R} O P H F R V s$ such that $\mathrm{F}\left(b_{t}\right) \leq \underline{\aleph}\left(b_{t}\right)$ and $\overline{\mathrm{F}}\left(b_{t}\right) \leq \bar{\aleph}\left(b_{t}\right)$. Then,

$$
\begin{aligned}
q-\operatorname{ROPHFRWG}\left(\mathrm{F}\left(b_{1}\right), \mathrm{F}\left(b_{2}\right), \ldots, \mathrm{F}\left(b_{n}\right)\right) \\
\quad \leq q-\operatorname{ROPHFRWG}\left(\aleph\left(b_{1}\right), \aleph\left(b_{2}\right), \ldots, \aleph\left(b_{n}\right)\right) .
\end{aligned}
$$

Proof.

(1) Idempotency: as $\mathcal{\aleph}\left(b_{t}\right)=\mathrm{F}(b)$ (for all $t=1,2,3, \ldots, n$ ) where $\mathrm{F}\left(b_{t}\right)=(\underline{\mathrm{F}}(b), \overline{\mathrm{F}}(b))=\left(\left(b_{h(x)} / \mathrm{\partial}_{h(x)}, d_{h(x)} /\right.\right.$ $\left.\left.\partial_{h(x)}\right),\left(\overline{b_{h(x)}} / \partial_{h(x)}, \overline{d_{h(x)}} / \overline{\partial_{h(x)}}\right)\right)$, it follows that 
$q-\operatorname{ROPHFREWG}\left(\aleph\left(b_{1}\right), \aleph\left(b_{2}\right), \ldots, \aleph\left(b_{n}\right)\right)$

$$
\begin{aligned}
& =\left(\stackrel{\otimes}{~}_{t=1}^{n}\left(\underline{\aleph}\left(b_{t}\right)\right)^{\gamma_{t}}, \stackrel{\otimes}{t}_{t=1}^{n}\left(\bar{\aleph}\left(b_{t}\right)\right)^{\gamma_{t}}\right)
\end{aligned}
$$

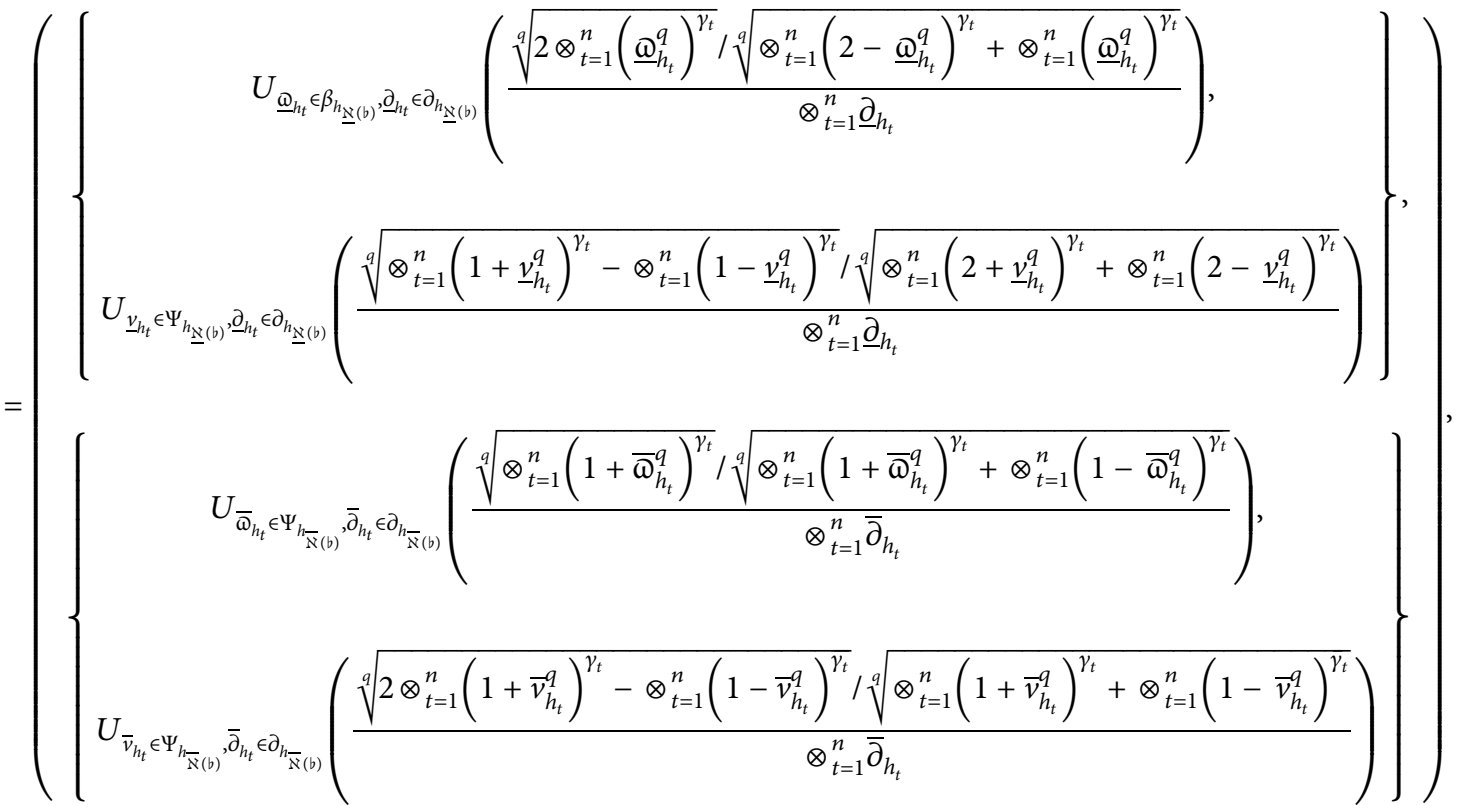

for all $t$, and $\aleph\left(b_{t}\right)=\mathrm{F}(b)=(\underline{F}(b)$,

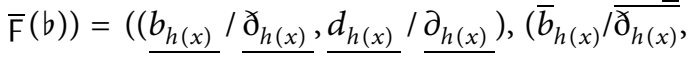

$\left.\overline{d_{h(x)}} / \overline{\left.\partial_{h(x)}\right)}\right)$. Therefore,

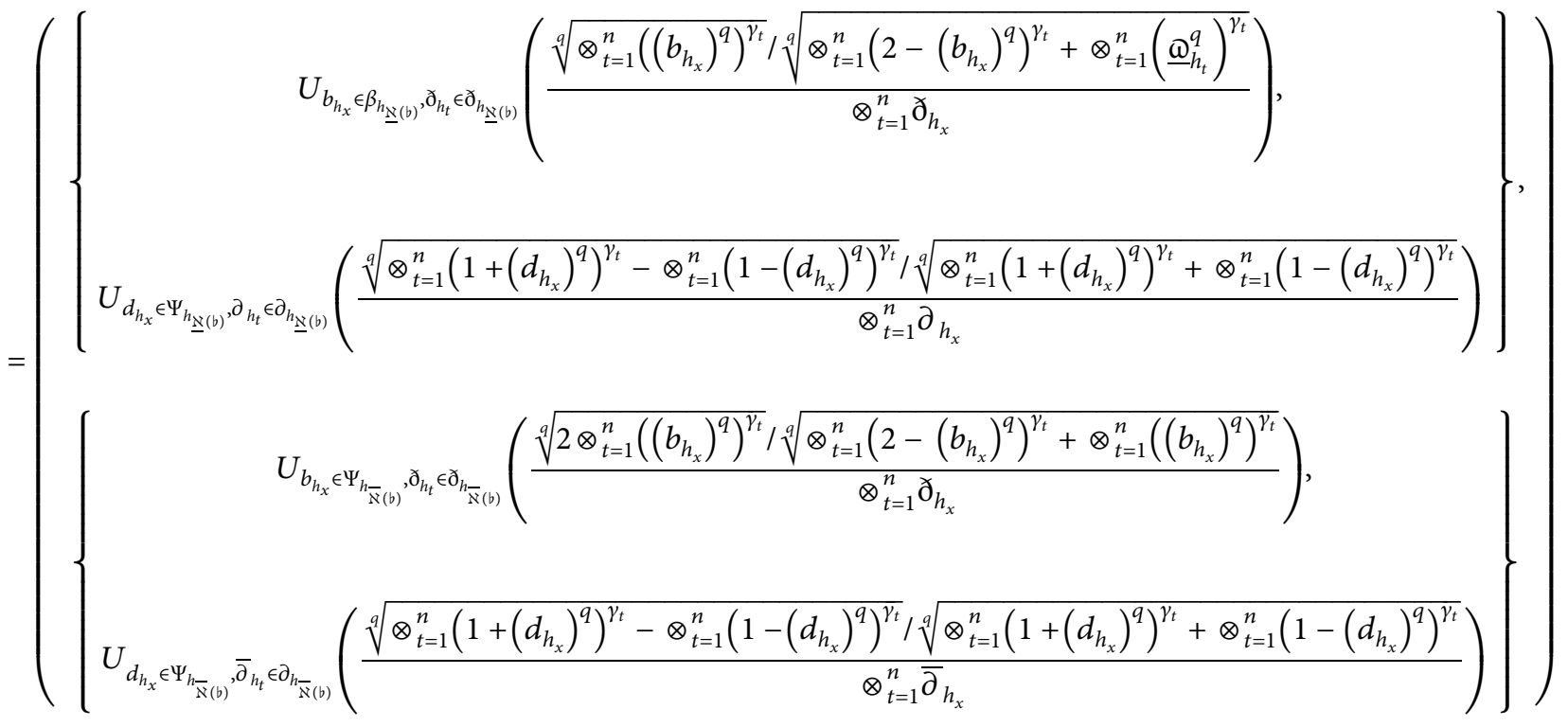

$$
\begin{aligned}
& =\left[\left(1-\left(1-\underline{b_{h(x)}} / \underline{\partial}_{h(x)}\right), \underline{d_{h(x)}} / \partial_{h(x)}\right),\left(1-\left(1-\bar{b}_{h(x)} / \bar{\partial}_{h(x)}\right), \bar{d}_{h(x)} / \overline{\partial_{h(x)}}\right)\right] \\
& =(\underline{F}(b), \bar{F}(b))=F(b) \text {. }
\end{aligned}
$$


Complexity

21

Hence,

$\left(\aleph\left(b_{1}\right), \aleph\left(b_{2}\right), \ldots, \aleph\left(b_{n}\right)\right)=F(b)$

(2) Boundedness:

$$
\begin{aligned}
& (\underline{\underline{N}}(b))^{-}=\left[\begin{array}{l}
\left(\min _{t}\left\{\frac{\Phi_{h_{t}}}{\overline{\partial_{h_{t}}}}\right\}, \max _{t}\left\{\frac{v_{h_{t}}}{\overline{\partial_{h_{t}}}}\right\}\right) \\
\left(\min _{t}\left\{\frac{\overline{\bar{\omega}_{h_{t}}}}{\overline{\partial_{h_{t}}}}\right\}, \max _{t}\left\{\frac{\overline{\nu_{h_{i}}}}{\overline{\partial_{h_{t}}}}\right\}\right)
\end{array}\right], \\
& (\underline{\underline{\aleph}}(b))^{+}=\left[\begin{array}{l}
\left(\max _{t}\left\{\frac{\Phi_{h_{t}}}{\overline{\partial_{h_{t}}}}\right\}, \min _{t}\left\{\frac{v_{h_{t}}}{\overline{\partial_{h_{t}}}}\right\}\right) \\
\left(\max _{t}\left\{\frac{\overline{\Phi_{h_{t}}}}{\overline{\partial_{h_{t}}}}\right\}, \min _{t}\left\{\frac{\overline{v_{h_{i}}}}{\overline{\partial_{h_{t}}}}\right\}\right)
\end{array}\right],
\end{aligned}
$$

$$
\begin{aligned}
(\aleph(b))^{-} & \leq q-\operatorname{ROPHFREWG}\left(\aleph\left(b_{1}\right), \aleph\left(b_{2}\right), \ldots, \aleph\left(b_{n}\right)\right) \\
& \leq(\aleph(b))^{+} .
\end{aligned}
$$

Let $g(x)=\sqrt[3]{2-x^{3} / x^{3}}, x \in(0,1] ; \quad$ then, $g^{\prime}(x)=-2 / \varkappa^{4} \sqrt[3]{\left(2-x^{3} / x^{3}\right)^{-2}}<0$. So, $g(x)$ is a decreasing function on $(0,1]$. Since $\left\{\underline{\omega_{h_{\min }}}\right\} \leq\left\{\underline{\omega_{h_{t}}}\right\} \leq\left\{\underline{\omega_{h_{\max }}}\right\} \quad$ for $\quad$ all $t$, $g\left(\underline{\omega_{h_{\max }}}\right) \leq \boldsymbol{g}\left(\underline{\left.\omega_{h_{t}}\right)} \leq \boldsymbol{g ( \omega _ { h _ { \operatorname { m i n } } }}\right)(t=1,2,3, \ldots, n)$, ie.,

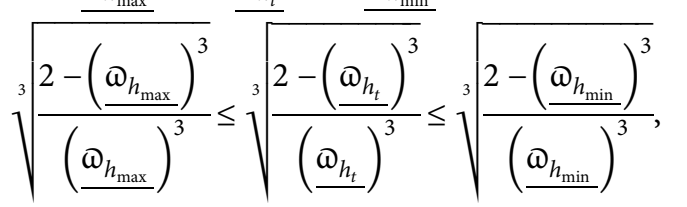

and let $\gamma=\left(\gamma_{1}, \gamma_{2}, \ldots, \gamma_{n}\right)^{T}$ be the weight vector such that $\left.\gamma_{t} \in 0,1\right]$ and $\oplus_{t=1}^{n} \gamma_{t}=1$ and $\partial_{h_{t}}$ be the probabilistic term such that $\oplus_{t=1}^{n} \partial_{h_{t}}=1$. We have

$$
\begin{aligned}
& \Leftrightarrow \frac{\sqrt[3]{\otimes_{t=1}^{n}\left(2-\left(\underline{\omega_{h_{\max }}}\right)^{3} /\left(\underline{\omega_{h_{\max }}^{3}}\right)\right)^{\gamma_{t}}}}{\otimes_{t=1}^{n} \underline{\partial_{h_{t}}}} \leq \frac{\sqrt[3]{\otimes_{t=1}^{n}\left(2-\left(\underline{\omega_{h_{t}}}\right)^{3} /\left(\underline{\omega_{h_{t}}^{3}}\right)\right)^{\gamma_{t}}}}{\otimes_{t=1}^{n} \underline{\partial_{h_{t}}}} \\
& \leq \frac{\sqrt[3]{\otimes_{t=1}^{n}\left(2-\left(\underline{\Phi_{h_{\max }}}\right)^{3} /\left(\underline{\omega_{h_{\max }}}\right)^{3}\right)^{\gamma_{t}}}}{\otimes_{t=1}^{n} \partial_{h_{t}}} \\
& \Leftrightarrow \frac{\sqrt[3]{\left(2-\left(\underline{\omega_{h_{\max }}}\right)^{3} /\left(\underline{\omega_{h_{\max }}}\right)^{3}\right)^{\oplus_{t=1}^{n} \gamma_{t}}}}{\otimes_{t=1}^{n} \underline{\partial_{h_{t}}}} \leq \frac{\sqrt[3]{\otimes_{t=1}^{n}\left(2-\left(\underline{\omega_{h_{t}}}\right)^{3} /\left(\underline{\omega_{h_{t}}}\right)^{3}\right)^{\gamma_{t}}}}{\otimes_{t=1}^{n} \underline{\partial_{h_{t}}}} \\
& \leq \frac{\sqrt[3]{\otimes_{t=1}^{n}\left(2-\left(\underline{\Phi_{h_{\max }}}\right)^{3} /\left(\underline{\omega_{h_{\max }}}\right)^{3}\right)^{\oplus_{t=1}^{n} \gamma_{t}}}}{\otimes_{t=1}^{n} \partial_{h_{t}}}, \\
& \Leftrightarrow \frac{\Phi_{h_{\min }}}{\otimes_{t=1}^{n} \underline{\partial_{h_{t}}}} \leq \frac{\sqrt[3]{\otimes_{t=1}^{n}\left(2-\left(\underline{\omega_{h_{t}}}\right)^{3} /\left(\underline{\Phi_{h_{t}}}\right)^{3}\right)^{\gamma_{t}}}}{\otimes_{t=1}^{n} \underline{\partial_{h_{t}}}} \\
& \leq \frac{\varpi_{h_{\max }}}{\otimes_{t=1}^{n} \partial_{h_{t}}} \\
& \Leftrightarrow \frac{\varpi_{h_{\min }}}{\partial_{h_{\min }}} \leq \frac{\sqrt[3]{\otimes_{t=1}^{n}\left(2-\left(\underline{\Phi_{h_{t}}}\right)^{3} /\left(\underline{\Phi_{h_{t}}}\right)^{3}\right)^{\gamma_{t}}}}{\otimes_{t=1}^{n} \underline{\partial_{h_{t}}}} \\
& \leq \frac{\varpi_{h_{\max }}}{\overline{\partial_{h_{\min }}}} .
\end{aligned}
$$


Similarly, we can show that

$$
\begin{aligned}
& \Leftrightarrow \frac{\overline{\omega_{h_{\min }}}}{\overline{\partial_{h_{\min }}}} \leq \frac{\sqrt[3]{\otimes_{t=1}^{n}\left(2-\left(\overline{\bar{\omega}_{h_{t}}}\right)^{3} /\left(\overline{\bar{\omega}_{h_{t}}}\right)^{3}\right)^{\gamma_{t}}}}{\otimes_{t=1}^{n} \overline{\partial_{h_{t}}}} \\
& \leq \frac{\overline{\widehat{\omega}_{h_{\max }}}}{\overline{\partial_{h_{\min }}}}
\end{aligned}
$$

Again, let $\left.f(y)=\sqrt[3]{1-y^{3} / 1+y^{3}}, y \in 0,1\right]$. Then, $f^{\prime}(y)=-2 y /\left(1+y^{3}\right)^{3} \sqrt[3]{\left(1-y^{3} / 1+y^{3}\right)^{-2}}<0$.

Thus, $f(y)$ is a decreasing function over $[0,1]$. Since $\left\{\underline{v_{h_{\max }}}\right\} \leq\left\{\underline{v_{h_{t}}}\right\} \leq\left\{\underline{v_{h_{\min }}}\right\} \quad$ for all $t$, $g\left(\underline{v_{h_{\min }}}\right) \leq \boldsymbol{g}\left(\nu_{h_{t}}\right) \leq \boldsymbol{g}\left(\underline{v_{h_{\max }}}\right)(t=1,2,3, \ldots, n)$, i.e.,

$$
\begin{aligned}
& \Leftrightarrow \sqrt[3]{\frac{1-\left(\underline{v_{h_{\min }}}\right)^{3}}{1+\left(\underline{v_{h_{\min }}}\right)^{3}}} \leq \sqrt[3]{\frac{1-\left(\underline{v_{h_{t}}}\right)^{3}}{1+\left(\underline{v_{h_{t}}}\right)^{3}}} \\
& \leq \sqrt[3]{\frac{1-\left(\underline{v_{h_{\max }}}\right)^{3}}{1+\left(\underline{v_{h_{\max }}}\right)^{3}}}, \quad t=1,2,3, \ldots, n,
\end{aligned}
$$

and let $\gamma=\left(\gamma_{1}, \gamma_{2}, \ldots, \gamma_{n}\right)^{T}$ be the weight vector such that $\left.\gamma_{t} \in 0,1\right]$ and $\oplus_{t=1}^{n} \gamma_{t}=1$ and $\partial_{h_{t}}$ be the probabilistic term such that $\oplus_{t=1}^{n} \partial_{h_{t}}=1$; $\frac{\partial_{l}}{\text { we have }}$

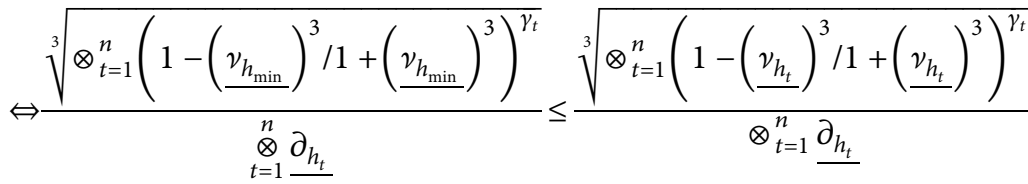

$$
\begin{aligned}
& \leq \frac{\sqrt[3]{\otimes_{t=1}^{n}\left(1-\left(\underline{v_{h_{\max }}}\right)^{3} / 1+\left(\underline{v_{h_{\max }}}\right)^{3}\right)^{\gamma_{t}}}}{\otimes_{t=1}^{n} \partial_{h_{t}}} \\
& \Leftrightarrow \frac{\sqrt[3]{\left(1-\left(\underline{v_{h_{\min }}}\right)^{3} / 1+\left(\underline{v_{h_{\min }}}\right)^{3}\right)^{\oplus_{t=1}^{n} \gamma_{t}}}}{\otimes_{t=1}^{n} \partial_{h_{t}}} \leq \frac{\sqrt[3]{\otimes_{t=1}^{n}\left(1-\left(\underline{v_{h_{t}}}\right)^{3} / 1+\left(\underline{v_{h_{t}}}\right)^{3}\right)^{\gamma_{t}}}}{\otimes_{t=1}^{n} \partial_{h_{t}}} \\
& \leq \frac{\sqrt[3]{\left(1-\left(\underline{v_{h_{\max }}}\right)^{3} / 1+\left(\underline{v_{h_{\max }}}\right)^{3}\right)^{\oplus_{t=1}^{n} \gamma_{t}}}}{\otimes_{t=1}^{n} \partial_{h_{t}}} \\
& \Leftrightarrow \frac{v_{h_{\max }}}{\otimes_{t=1}^{n} \partial_{h_{t}}} \leq \frac{\sqrt[3]{\otimes_{t=1}^{n}\left(1-\left(\underline{v_{h_{t}}}\right)^{3} / 1+\left(\underline{v_{h_{t}}}\right)^{3}\right)^{\gamma_{t}}}}{\otimes_{t=1}^{n} \underline{\partial_{h_{t}}}} \\
& \leq \frac{v_{h_{\min }}}{\otimes_{t=1}^{n} \partial_{h_{t}}}, \\
& \Leftrightarrow \frac{v_{h_{\max }}}{\underline{\partial_{h_{\max }}}} \leq \frac{\sqrt[3]{\otimes_{t=1}^{n}\left(1-\left(\underline{v_{h_{t}}}\right)^{3} / 1+\left(\underline{v_{h_{t}}}\right)^{3}\right)^{\gamma_{t}}}}{\otimes_{t=1}^{n} \underline{\partial_{h_{t}}}} \\
& \leq \underline{\underline{\nu_{h_{\text {min }}}}} .
\end{aligned}
$$

In a similar way, we can show that 


$$
\begin{aligned}
& \Leftrightarrow \frac{\overline{v_{h_{\max }}}}{\overline{\partial_{h_{\max }}}} \leq \frac{\sqrt[3]{\otimes_{t=1}^{n}\left(2-\left(\overline{v_{h_{t}}}\right)^{3} /\left(\overline{v_{h_{t}}}\right)^{3}\right)^{\gamma_{t}}}}{\otimes_{t=1}^{n} \partial_{h_{t}}} \\
& \leq \frac{\overline{v_{h_{\min }}}}{\partial_{h_{\min }}}
\end{aligned}
$$

Thus, from equations (70), (71), (74), and (75), we have

$$
\begin{aligned}
(\aleph(b))^{-} & \leq q-\operatorname{ROPHFREWG}\left(\aleph\left(b_{1}\right), \aleph\left(b_{2}\right), \ldots, \aleph\left(b_{n}\right)\right) \\
& \leq(\aleph(b))^{+} .
\end{aligned}
$$

(3) Monotonicity: the proof is similar to the proof of (2).

\section{Multiattribute Decision- Making Methodology}

Herein, we develop an algorithm for addressing uncertainty in MAGDM under $q$-ROHFR information. Consider a DM problem with a set $\left\{A_{1}, A_{2}, \ldots, A_{n}\right\}$ of $n$ alternatives and a set of $n$ attributes $\left\{\chi_{1}, \chi_{2}, \ldots, \chi_{n}\right\}$ with $\left(\gamma_{1}, \gamma_{2}, \ldots, \gamma_{n}\right)^{T}$ being the weight vector; that is, $\left.\gamma_{t} \in 0,1\right], \oplus_{t=1}^{n} \gamma_{t}=1$. Also, $\partial_{h_{t}}$ and $\partial_{h_{t}}$ are probabilistic terms such that $\oplus_{t=1}^{n} \partial_{h_{t}}=1$ and $\oplus_{t=1}^{n} \partial_{h_{t}}=1$ with the property that $0 \leq \partial_{h_{t}}$ and $\partial_{h_{t}} \leq 1$. To test the reliability of $k$ th alternative $A_{t}$ under the attribute $c_{t}$, let $\left\{D_{1}, D_{2}, \ldots, D_{\hat{J}}\right\}$ be a set of decision makers (DMs). The expert evaluation matrix is described as

$$
\begin{aligned}
M & =\left[\aleph\left(b_{t j}^{\hat{J}}\right)\right]_{m \times n} \\
& =\left[\begin{array}{cccc}
\left(\underline{\aleph}\left(b_{11}\right), \underline{\aleph}\left(b_{11}\right)\right) & \left(\underline{\aleph}\left(b_{12}\right), \underline{\aleph}\left(b_{12}\right)\right) & \cdots & \left(\underline{\aleph}\left(b_{1 j}\right), \bar{\aleph}\left(b_{1 j}\right)\right) \\
\left(\underline{\aleph}\left(b_{21}\right), \bar{\aleph}\left(b_{21}\right)\right) & \left(\bar{\aleph}\left(b_{22}\right), \bar{\aleph}\left(b_{22}\right)\right) & \cdots & \left(\bar{\aleph}\left(b_{2 j}\right), \bar{\aleph}\left(b_{2 j}\right)\right) \\
\left.\left(b_{31}\right), \bar{\aleph}\left(b_{31}\right)\right) & \left(\underline{\aleph}\left(b_{32}\right), \bar{\aleph}\left(b_{32}\right)\right) & \cdots & \left(\underline{\aleph}\left(b_{3 j}\right), \bar{\aleph}\left(b_{3 j}\right)\right) \\
\vdots & \vdots & \ddots & \vdots \\
\left(\underline{\aleph}\left(b_{t 1}\right), \bar{\aleph}\left(b_{t 1}\right)\right) & \left(\underline{\aleph}\left(b_{t 2}\right), \bar{\aleph}\left(b_{t 2}\right)\right) & \cdots & \left(\underline{\aleph}\left(b_{t j}\right), \bar{\aleph}\left(b_{t j}\right)\right)
\end{array}\right],
\end{aligned}
$$

where

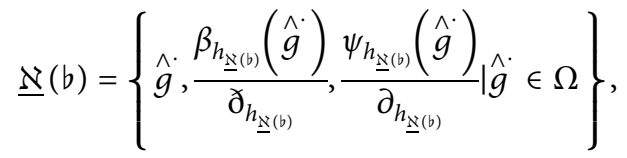

$$
\begin{aligned}
& \bar{\aleph}\left(b_{t j}\right)=\left\{\stackrel{\wedge}{g}, \frac{\beta_{h_{\underline{\underline{N}}(b)}}(\stackrel{\wedge}{g})}{\partial_{h_{\underline{\underline{N}}(b)}}}, \frac{\psi_{h_{\underline{\underline{\aleph}}(b)}}(\stackrel{\wedge}{g})}{\partial_{h_{\underline{\underline{N}}(b)}}} \mid \stackrel{\wedge}{g} \in \Omega\right\},
\end{aligned}
$$

such that

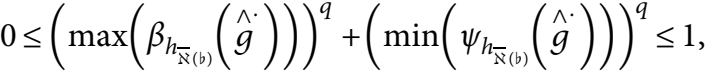

$$
\begin{aligned}
& 0 \leq\left(\min \left(\beta_{h_{\underline{\mathrm{N}}(b)}}(\stackrel{\wedge}{g})\right)^{q}+\left(\max \left(\psi_{h_{\underline{\mathrm{N}}(b)}}(\stackrel{\wedge}{g})\right)\right)^{q} \leq 1\right.
\end{aligned}
$$

are the q-ROPHFR values. The main steps for MAGDM are as follows.

Construct the experts' evaluation matrices as

$$
(E)^{\hat{J}}=\left[\begin{array}{cccc}
\left(\underline{\aleph}\left(b_{11}^{\hat{J}}\right), \bar{\aleph}\left(b_{11}^{\hat{J}}\right)\right) & \left(\underline{\aleph}\left(b_{12}^{\hat{J}}\right), \bar{\aleph}\left(b_{12}^{\hat{J}}\right)\right) & \ldots & \left(\bar{\aleph}\left(b_{1 j}^{\hat{J}}\right), \bar{\aleph}\left(b_{1 j}^{\hat{J}}\right)\right) \\
\left.\left(b_{21}^{\hat{J}}\right), \bar{\aleph}\left(b_{21}^{\hat{J}}\right)\right) & \left(\underline{\aleph}\left(b_{22}^{\hat{J}}\right), \bar{\aleph}\left(b_{22}^{\hat{J}}\right)\right) & \ldots & \left(\underline{\aleph}\left(b_{2 j}^{J}\right), \bar{\aleph}\left(b_{2 j}^{J}\right)\right) \\
\vdots & \ldots & \vdots \\
\left(\underline{\aleph}\left(b_{t 1}^{\hat{J}}\right), \bar{\aleph}\left(b_{t 1}^{\hat{J}}\right)\right) & \left(\underline{\aleph}\left(b_{t 2}^{\hat{J}}\right), \bar{\aleph}\left(b_{t 2}^{\hat{J}}\right)\right) & \ldots & \left(\bar{\aleph}\left(b_{t j}^{\hat{J}}\right), \bar{\aleph}\left(b_{t j}^{\hat{J}}\right)\right)
\end{array}\right],
$$

where $\hat{J}$ shows the number of experts. 
Evaluate the normalized experts' matrices $(N)^{\hat{J}}$ as

$$
(N)^{\hat{J}}= \begin{cases}\aleph\left(b_{t j}\right)=\left(\underline{\aleph}\left(b_{t j}\right), \bar{\aleph}\left(b_{t j}\right)\right) & \text { if for the benefit type, } \\ \left(\aleph\left(b_{t j}\right)\right)^{c}=\left(\left(\underline{\aleph}\left(b_{t j}\right)\right)^{c},\left(\bar{\aleph}\left(b_{t j}\right)\right)^{c}\right) & \text { if for the cost type. }\end{cases}
$$

The weight information of the attributes is determined by using the Shannon entropy measure in the following way. The entropy measure corresponding to each attribute is

$$
\begin{aligned}
\operatorname{EN}\left(\aleph_{j}\right) & =\operatorname{EN}\left(\aleph_{1 j}, \aleph_{2 j}, \aleph_{3 j}, \ldots, \aleph_{t j}\right) \\
& =\frac{-1}{\ln (n)} \sum_{t=1}^{n}\left(\underline{\omega_{h_{t j}}} \ln \left(\underline{\Phi_{h_{t j}}}\right) \times \underline{\partial_{t}}+\overline{\bar{\omega}_{h_{t j}}} \ln \left(\overline{\Phi_{h_{t j}}}\right) \times \overline{\partial_{h_{t}}}+\underline{v_{h_{t j}}} \ln \left(\underline{v_{h_{t j}}}\right) \times \underline{\partial_{h_{t}}}+\overline{\nu_{h_{t j}}} \ln \left(\overline{v_{h_{t j}}}\right) \times \overline{\partial_{h_{t}}}\right), \quad j=1,2,3, \ldots, m .
\end{aligned}
$$

Then,

$$
\gamma\left(\aleph_{j}\right)=\frac{1-\operatorname{EN}\left(\aleph_{j}\right)}{\sum_{j=1}^{m} 1-\operatorname{EN}\left(\aleph_{j}\right)}
$$

Thus, weights of attributes are found as $\gamma\left(\aleph_{j}\right)=\left(\gamma\left(\aleph_{1}\right), \gamma\left(\aleph_{2}\right), \ldots, \gamma\left(\aleph_{n}\right)\right)^{T}$.

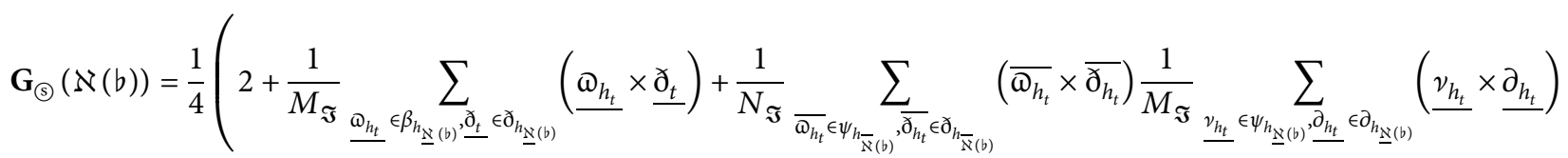

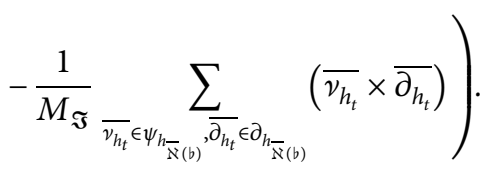

Rank all the alternative scores in the descending order. The alternative having a larger value will be superior/best.

The algorithm steps/flowchart of the decision-making technique are shown in Figure 3.

\section{Numerical Example}

To strengthen our developed operators, we consider a numerical MCGDM example of drug selection for the treatment of COVID-19.

8.1. Case Study (Drug Selection for the Treatment of COVID19). Nowadays, an overwhelming majority of the world is fighting against an epidemic called coronavirus. Coronavirus is a new virus that has recently been identified in
Compute the $q$-ROPHFRVs for each considered alternative with respect to the given list of criteria/attributes by utilizing the proposed aggregation information.

Find the ranking of alternatives based on the score function as 


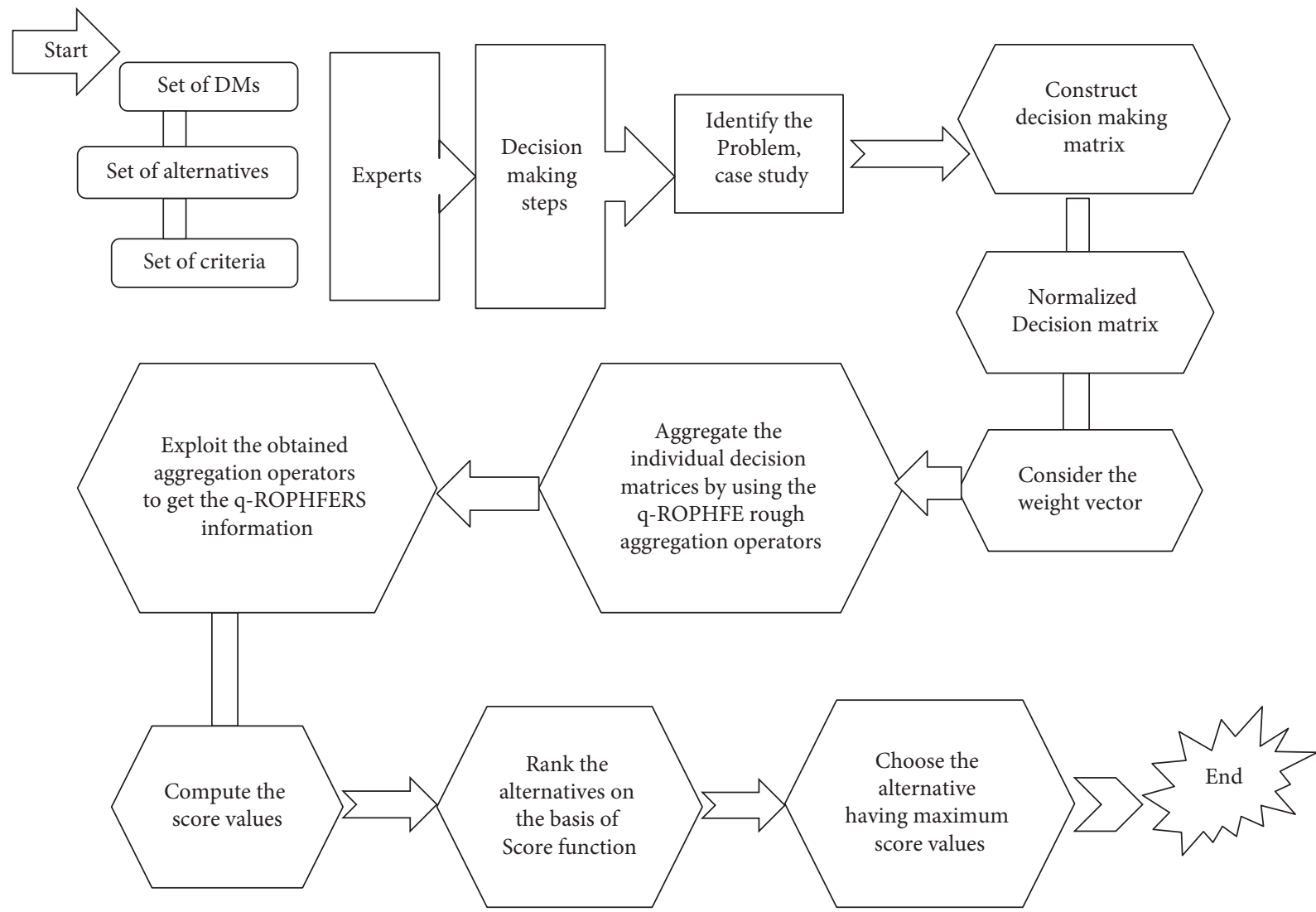

FIGURE 3: Algorithm steps for decision-making.

could be effective against COVID-19. The combination of lopinavir/ritonavir and interferon-beta (LPV/RTV-IFNb) reduced viral masses slightly without impacting other clinical factors. Remdesivir (GS-5734), a nucleotide analog prodrug, was earlier tested for SARS, MERS, and Ebola [67]. Remdesivir has been demonstrated to be safe and beneficial for patients with mild COVID-19 symptoms, according to an experimental investigation [68]. Hydroxychloroquine (HCQ) and chloroquine (CQ) are widely used antimalarial drugs that stimulate immunomodulatory responses and also used to avoid autoimmune disorders. Wang et al. [69] testified that HCQ was found to be more stimulating than CQ in vitro. The potential effectiveness of these drugs in regulating cytokine discharge syndrome in patients has been investigated from the global pandemic of COVID-19. Even though there is no effective treatment for COVID-19, all antiviral medicines should be investigated further in clinical testing. According to a WHO report released on June 13, 2021, more than 176,396,104 cases of COVID-19 have been reported worldwide, resulting in more than 3,810,989 deaths. A total of 160,398,032 people have been recovered [2]. The most common symptoms and signs reported by COVID-19 patients are fever (83\%-99\%), shortness of breath $(31 \%-40 \%)$, fatigue $(44 \%-70 \%)$, anorexia $(40 \%-$ $84 \%)$, cough $(59 \%-82 \%)$, sputum production $(28 \%-33 \%)$, and myalgia $(11 \%-35 \%)$ [70-74].

Here, we proposed four medicines as alternatives for the treatment of COVID-19 patients, namely, LPV/RTV-IFNb
$\left(A_{1}\right)$, remdesivir $\left(A_{2}\right), \operatorname{LPV} / \operatorname{RTV}\left(A_{3}\right)$, and favipiravir $\left(A_{4}\right)$. Antiviral medications should be selected not only for their effect on symptoms but also for their effectiveness and possible side effects. Therefore, we take four parameters, cough $\left(\chi_{1}\right)$, fatigue $\left(\chi_{2}\right)$, fever $\left(\chi_{3}\right)$, and shortness of breath $\left(\chi_{4}\right)$. For selection of optimal medicine, information is presented as $q$-ROPHFR information. The corresponding weight vector for criteria is $\gamma=(0.13,0.27,0.29,0.31)^{T}$. The following computations are performed to address the MCDM problem using the established methodology for evaluating alternatives.

The information of the professional expert is given in Tables 1 and 2 in the form of q-ROPHFRS.

The expert information is of the benefit type. So, in this case, we do not need to normalize the $q$-ROPHFRVs.

In this problem, only one expert is considered for collection of uncertain information. So, we do not need to find the collected information.

Aggregation information of the alternative under the given list of attributes is evaluated using proposed aggregation operators which are as follows.

Case 1. Aggregation information using the EWA operator is shown in Table 3.

Case 2. Aggregation information using the $q$-ROHFREWG operator is presented in Table 4. 
TABLE 1: Expert information.

\begin{tabular}{lcc}
\hline & $\chi_{1}$ & $\chi_{2}$ \\
\hline$A_{1}$ & $\left(\begin{array}{c}((0.1 / 0.3,0.2 / 0.5,0.5 / 0.2)(0.3 / 0.6,0.4 / 0.4)) \\
,((0.8 / 1)(0.4 / 0.5,0.6 / 0.5))\end{array}\right)$ & $\left(\begin{array}{c}((0.5 / 0.4,0.7 / 0.6)(0.5 / 0.7,0.6 / 0.3)) \\
,((0.4 / 0.5,0.6 / 0.5)(0.7 / 0.3,0.9 / 0.7))\end{array}\right)$ \\
$A_{2}$ & $\left(\begin{array}{c}((0.6 / 0.7,0.7 / 0.3)(0.7 / 0.5,0.9 / 0.5)) \\
,((0.3 / 0.2,0.5 / 0.8)(0.6 / 1))\end{array}\right)$ & $\left(\begin{array}{c}((0.2 / 0.2,0.4 / 0.1,0.5 / 0.7)(0.5 / 1.0)) \\
,((0.6 / 0.3,0.7 / 0.7)(0.3 / 1))\end{array}\right)$ \\
$A_{3}$ & $\left(\begin{array}{c}((0.4 / 0.3,0.5 / 0.6,0.6 / 0.1)(0.6 / 0.1,0)(0.5 / 0.5,0.6 / 0.5)) \\
((0.9 / 1)(0.5 / 1))\end{array}\right)\left(\begin{array}{c}((0.4 / 0.3,0.6 / 0.4,0.7 / 0.3)(0.5 / 0.2,0.7 / 0.8)) \\
A_{4}\end{array}\right.$ & $\left(\begin{array}{c}((0.4 / 0.4,0.5 / 0.6)(0.4 / 1)) \\
,((0.1 / 0.6,0.2 / 0.4)(0.2 / 0.2,0.3 / 0.8))\end{array}\right)$ \\
\hline
\end{tabular}

TABLE 2: Expert information.

\begin{tabular}{|c|c|c|}
\hline & $\chi_{3}$ & $\chi_{4}$ \\
\hline$A_{1}$ & $\left(\begin{array}{c}((0.4 / 1),(0.3 / 0.2,0.7 / 0.8)), \\
((0.5 / 1),(0.9 / 1))\end{array}\right)$ & $\left(\begin{array}{c}((0.6 / 1),(0.7 / 1)), \\
((0.6 / 0.4,0.8 / 0.2,0.9 / 0.4),(0.7 / 0.7,0.9 / 0.3))\end{array}\right)$ \\
\hline$A_{2}$ & $\left(\begin{array}{c}((0.8 / 1),(0.4 / 0.6,0.5 / 0.2,0.7 / 0.2)) \\
((0.2 / 0.6,0.5 / 0.4),(0.4 / 0.3,0.5 / 0.7))\end{array}\right)$ & $\left(\begin{array}{c}((0.8 / 1),(0.5 / 1)), \\
((0.7 / 1.0),(0.1 / 0.5,0.3 / 0.3,0.4 / 0.2))\end{array}\right)$ \\
\hline$A_{3}$ & $\left(\begin{array}{c}((0.3 / 1),(0.7 / 0.6,0.8 / 0.4)), \\
((0.7 / 0.6,0.8 / 0.4),(0.1 / 0.7,0.4 / 0.2,0.7 / 0.1))\end{array}\right)$ & $\left(\begin{array}{c}((0.3 / 0.2,0.6 / 0.8),(0.8 / 1)), \\
((0.7 / 1),(0.3 / 1))\end{array}\right)$ \\
\hline$A_{4}$ & $\left(\begin{array}{c}((0.3 / 1),(0.7 / 0.7,0.8 / 0.3)), \\
((0.7 / 1),(0.6 / 1))\end{array}\right)$ & $\left(\begin{array}{c}((0.6 / 0.2,0.7 / 0.4,0.9 / 0.4),(0.3 / 0.3,0.4 / 0.7)) \\
((0.2 / 0.2,0.7 / 0.8),(0.7 / 0.4,0.8 / 0.2,0.9 / 0.4))\end{array}\right)$ \\
\hline
\end{tabular}

TABLE 3: Aggregated information using $q$-ROPHFREWA.

$A_{1}$

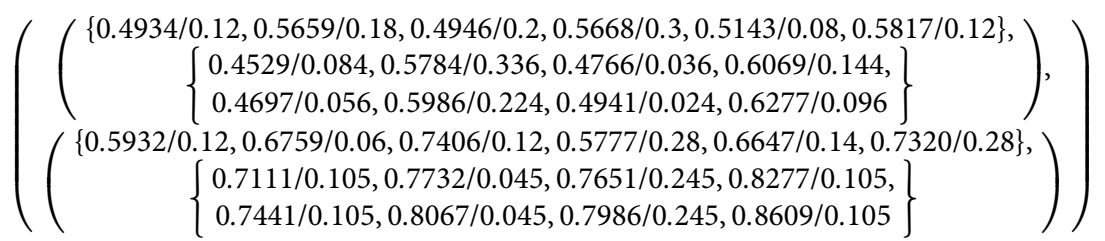

$A_{2}$

$\left.\left(\begin{array}{c}\left\{\begin{array}{c}\{0.7074 / 0.14,0.7160 / 0.07,0.7252 / 0.49,0.7074 / 0.06,0.7160 / 0.03,0.7252 / 0.21\} \\ \{0.4913 / 0.03,0.5234 / 0.1,0.5787 / 0.1,0.5120 / 0.3,0.5452 / 0.1,0.6021 / 0.1\}\end{array}\right) \\ \left\{\begin{array}{c}0.5584 / 0.036,0.5915 / 0.024,0.5944 / 0.084,0.6235 / 0.056 \\ 0.5714 / 0.144,0.6030 / 0.096,0.6057 / 0.336,0.6336 / 0.224\end{array}\right\}, \\ \{0.2555 / 0.15,0.3579 / 0.09,0.3911 / 0.06,0.2733 / 0.35,0.3824 / 0.21,0.4176 / 0.21\}\end{array}\right)\right)$

$A_{3}$

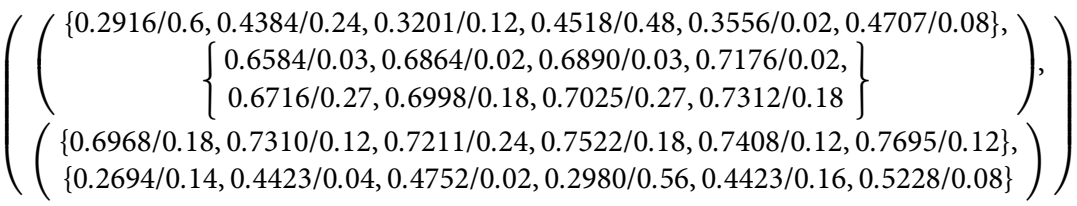

$A_{4}$

$\left.\left(\begin{array}{c}\left(\begin{array}{c}\{0.4659 / 0.08,0.5233 / 0.16,0.6769 / 0.16,0.4899 / 0.12,0.5424 / 0.24,0.6876 / 0.24\} \\ \left\{\begin{array}{l}0.4475 / 0.105,0.4879 / 0.245,0.4689 / 0.045,0.5109 / 0.105, \\ 0.4586 / 0.105,0.4999 / 0.245,0.4805 / 0.045,0.5233 / 0.105\end{array}\right\}\end{array}\right), \\ \left\{\begin{array}{l}0.4784 / 0.084,0.5990 / 0.336,0.4811 / 0.056,0.6006 / 0.224, \\ 0.4852 / 0.036,0.6032 / 0.144,0.4879 / 0.024,0.6048 / 0.144\end{array}\right\}, \\ \{0.4967 / 0.08,0.5217 / 0.04,0.5478 / 0.08,0.5503 / 0.32,0.5773 / 0.16,0.6052 / 0.32\}\end{array}\right)\right)$


Score values of all alternatives under developed AOPs are presented in Table 5.

Rank the alternatives $A_{t}(t=1,2, \ldots, 4)$ which is enclosed in Table 6.

From the above computations, we concluded that alternative $A_{2}$ is the finest alternative among others, and therefore, it is highly recommended (Figure 4).

\section{Reliability and Validity Test}

In practice, selecting the perfect option from the group's decision matrices is a challenging task. The approach for analyzing the reliability and validity of DM systems was developed by Wang and Garg [75]. The testing procedure is as follows:

Test step 1: if we substitute the normalized element for the worse element of the alternative by presenting the appropriate alternative with no modification and also with no altering the comparable position of each decision criterion, the appropriate and effective MAGDM technique is to do so.

Test step 2: the transitive property must be satisfied using an efficient and appropriate MAGDM approach.

Test step 3: when a MAGDM problem is reduced to a minor one, a combined alternative rating should be similar to the original rating of the undecomposed problem. To rank the alternative, we utilize the same methods adopted in the MAGDM problem on minor issues. The MAGDM problem was reduced to a smaller one in order to achieve the best result, and the same suggested DM technique has been used. The appropriate and effective MAGDM technique is that if we apply the same procedure to a small problem, the result would be the same as the MAGDM problem.

9.1. Validity Test for the Proposed DM Methodology. Utilizing the competency of the aforementioned test, we check the appropriation and validation of our established approach (Tables 7 and 8). The $q$-ROPHFR information is enclosed in Tables 9 and 10 as follows:

Test step 1: we substitute the worse element of the alternative by presenting the appropriate alternative with no modification and also with no altering the comparable position of each decision criterion, in this step. Table 11 encloses the updated decision matrix.

Now, we calculate the combined values of each alternative under criteria weight $(0.13,0.27,0.29,0.31)^{T}$ using the proposed list of $q$-rung orthopair probabilistic hesitant fuzzy rough aggregation operators as follows:

Case I: aggregated information using $q$-ROPHFRWA operators is enclosed in Table 12.

Case II: aggregated information using $q$-ROPHFRWG operators is enclosed in Table 13.

Score values of all alternatives under developed aggregation operators are presented in Table 13.

Rank the alternatives $A_{t}(t=1,2, \ldots, 4)$ which is enclosed in Table 14.

We get again the same alternative $A_{2}$ by using test step 1 , which is also obtained by applying our proposed method.

We are now testing the validity test steps 2 and 3 to demonstrate that the proposed approach is reliable and relevant. To this end, we first transformed the MAGDM problem into three smaller subproblems such as $\left\{A_{2}, A_{1}, A_{4}\right\}$, $\left\{A_{1}, A_{4}, A_{3}\right\}$, and $\left\{A_{2}, A_{4}, A_{3}\right\}$. We now implement our suggested decision-making approach to the smaller problems that have been transformed and give us the ranking of alternatives as $A_{2}>A_{1}>A_{4}, A_{1}>A_{4}>A_{3}$, and $A_{2}>A_{4}>A_{3}$, respectively. We analyzed that $A_{2}>A_{1}>A_{4}>A_{3}$ is the same as the standard decision-making approach results when assigning detailed ranking (Figure 5).

\section{Comparison Analysis}

10.1. TOPSIS Methodology Based on q-Rung Orthopair Probabilistic Hesitant Fuzzy Rough Information. Hwang and Yoon proposed the TOPSIS technique for the ideal solution, which allows policymakers to compare the PIS and NIS. TOPSIS is based on the assumption that the best alternative would be the closest to the ideal and the furthest away from the perfect negative solution $[76,77]$. The main parts of the method are as follows.

Let $b=\left\{A_{1}, A_{2}, A_{3}, \ldots, A_{n}\right\}$ be the set of alternatives and $C=\left\{\chi_{1}, \chi_{2}, \chi_{3}, \ldots, \chi_{n}\right\}$ be a set of criteria. The decision matrix of the expert is presented as

$$
\begin{aligned}
& M=\left[\underline{\aleph}\left(b_{t j}^{\hat{J}}\right), \bar{\aleph}\left(\hat{b}_{t j}^{\hat{J}}\right)\right]_{m \times n}
\end{aligned}
$$

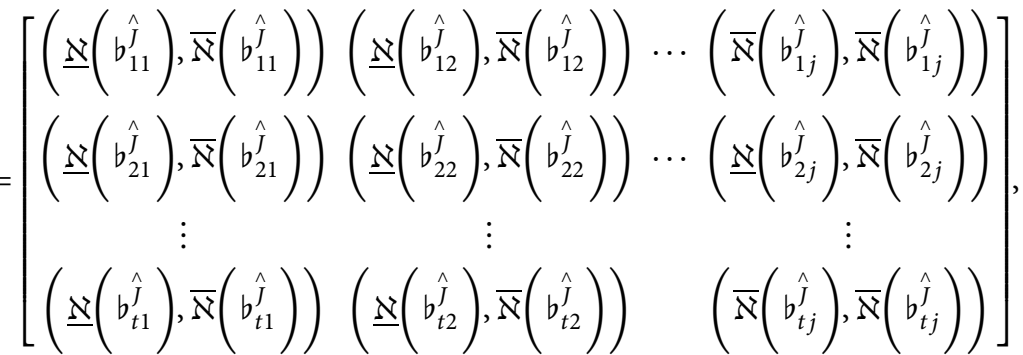


TABLE 4: Aggregated information using $q$-ROPHFREWG.

$A_{1}$

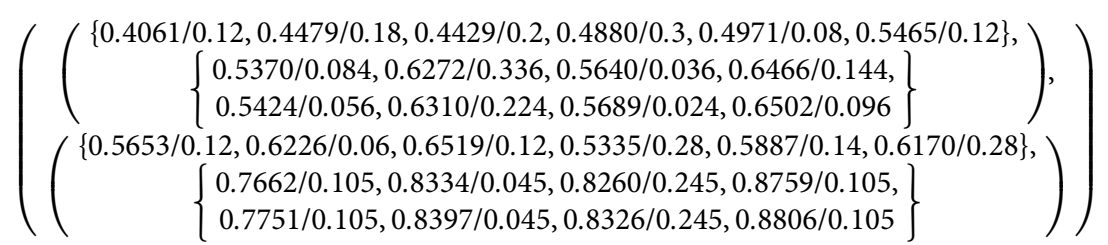

$A_{2}$ $\left(\begin{array}{c}\left(\begin{array}{c}\{0.5492 / 0.14,0.6505 / 0.07,0.6864 / 0.49,0.5492 / 0.06,0.6505 / 0.03,0.6864 / 0.21\} \\ \{0.5156 / 0.03,0.5366 / 0.1,0.6031 / 0.1,0.5933 / 0.3,0.6090 / 0.1,0.6608 / 0.1\}\end{array}\right) \\ \left\{\begin{array}{c}\left\{\begin{array}{l}0.4256 / 0.036,0.5497 / 0.024,0.4458 / 0.084,0.5744 / 0.056 \\ 0.4545 / 0.144,0.5851 / 0.096,0.4759 / 0.336,0.6108 / 0.224\end{array}\right\}, \\ \{0.3795 / 0.15,0.3973 / 0.09,0.4201 / 0.06,0.4169 / 0.35,0.4317 / 0.21,0.4512 / 0.21\}\end{array}\right)\end{array}\right)$

$A_{3}$ $\left(\left\{\begin{array}{c}0.2320 / 0.6,0.2900 / 0.24,0.2391 / 0.12, \\ 0.2988 / 0.48,0.2453 / 0.02,0.3066 / 0.08\end{array}\right\},\left\{\begin{array}{c}0.6902 / 0.03,0.7252 / 0.02,0.7055 / 0.03,0.7386 / 0.02, \\ 0.7011 / 0.27,0.7347 / 0.18,0.7158 / 0.27,0.7476 / 0.18\end{array}\right\}\right)$ $\left(\left\{\begin{array}{c}0.6314 / 0.18,0.6589 / 0.12,0.6976 / 0.24, \\ 0.7263 / 0.18,0.7258 / 0.12,0.7548 / 0.12\end{array}\right\},\left\{\begin{array}{c}0.3890 / 0.14,0.5179 / 0.04,0.5439 / 0.02, \\ 0.4945 / 0.56,0.5179 / 0.16,0.6048 / 0.08\end{array}\right\}\right)$

$A_{4}$ $\left(\begin{array}{c}\left(\begin{array}{c}\{0.4193 / 0.08,0.4422 / 0.16,0.4888 / 0.16,0.4455 / 0.12,0.4697 / 0.24,0.5185 / 0.24\} \\ 0.5249 / 0.105,0.5382 / 0.245,0.5878 / 0.045,0.5983 / 0.105, \\ 0.5390 / 0.105,0.5515 / 0.245,0.5989 / 0.045,0.6089 / 0.105\end{array}\right\}\end{array}\right)$,

TABle 5: Score values.

\begin{tabular}{lcccc}
\hline Operators & $\mathbf{G}_{\odot}\left(A_{1}\right)$ & $\mathbf{G}_{\Im}\left(A_{2}\right)$ & $\mathbf{G}_{\odot}\left(A_{3}\right)$ & 0.5173 \\
\hline q-ROPHFREWA & 0.5078 & 0.5176 & $\mathbf{G}_{\Im}\left(A_{4}\right)$ \\
q-ROPHFREWG & 0.4989 & 0.5066 & 0.5018 & 0.5044 \\
\hline
\end{tabular}

TABLE 6: Ranking of the alternatives.

\begin{tabular}{lll}
\hline Operators & Score & Best alternative \\
\hline q-ROPHFREWA & $\mathbf{G}_{\odot}\left(A_{2}\right)>\mathbf{G}_{\odot}\left(A_{3}\right)>\mathbf{G}_{\odot}\left(A_{1}\right)>\mathbf{G}_{\odot}\left(A_{4}\right)$ & $A_{2}$ \\
q-ROPHFREWG & $\mathbf{G}_{\odot}\left(A_{2}\right)>\mathbf{G}_{\odot}\left(A_{3}\right)>\mathbf{G}_{\odot}\left(A_{1}\right)>\mathbf{G}_{\odot}\left(A_{4}\right)$ & $A_{2}$ \\
\hline
\end{tabular}

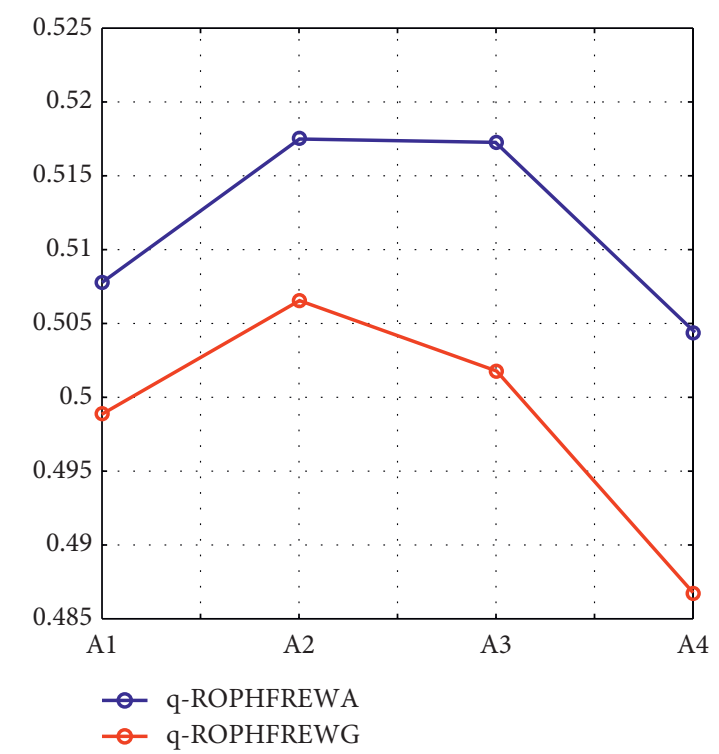

Figure 4: Alternatives using EWA and EWG operators. 
TABLE 7: Expert information.

\begin{tabular}{|c|c|c|}
\hline & $\chi_{1}$ & $\chi_{2}$ \\
\hline$A_{1}$ & $\left(\begin{array}{c}((0.1 / 0.3,0.2 / 0.5,0.5 / 0.2),(0.3 / 0.6,0.4 / 0.4)), \\
((0.8 / 1),(0.4 / 0.5,0.6 / 0.5))\end{array}\right)$ & $\left(\begin{array}{c}((0.5 / 0.4,0.7 / 0.6),(0.5 / 0.7,0.6 / 0.3)), \\
((0.4 / 0.3,0.5 / 0.7),(0.7 / 0.3,0.9 / 0.7))\end{array}\right)$ \\
\hline$A_{2}$ & $\left(\begin{array}{c}((0.6 / 0.7,0.7 / 0.3),(0.7 / 0.5,0.9 / 0.5)) \\
((0.3 / 0.2,0.5 / 0.8),(0.6 / 1))\end{array}\right)$ & $\left(\begin{array}{c}((0.2 / 0.2,0.4 / 0.1,0.5 / 0.7),(0.5 / 1.0)), \\
((0.6 / 0.3,0.7 / 0.7),(0.3 / 1))\end{array}\right)$ \\
\hline$A_{3}$ & $\left(\begin{array}{c}((0.4 / 0.3,0.5 / 0.6,0.6 / 0.1),(0.6 / 0.1,0.7 / 0.9)), \\
((0.9 / 1),(0.5 / 1))\end{array}\right)$ & $\left(\begin{array}{c}((0.1 / 1.0),(0.5 / 0.5,0.6 / 0.5)) \\
((0.4 / 0.3,0.6 / 0.4,0.7 / 0.3),(0.5 / 0.2,0.7 / 0.8))\end{array}\right.$ \\
\hline$A_{4}$ & $\left(\begin{array}{c}((0.4 / 1),(0.5 / 0.5,0.6 / 0.5)) \\
((0.3 / 0.7,0.4 / 0.3),(0.8 / 1))\end{array}\right)$ & $\left(\begin{array}{c}((0.4 / 0.4,0.5 / 0.6),(0.4 / 1)), \\
((0.1 / 0.6,0.2 / 0.4),(0.2 / 0.2,0.3 / 0.8))\end{array}\right)$ \\
\hline
\end{tabular}

TABle 8: Expert information.

\begin{tabular}{lcc}
\hline & $\chi_{3}$ & $\chi_{4}$ \\
\hline$A_{1}$ & $\left(\begin{array}{c}((0.4 / 1),(0.3 / 0.2,0.7 / 0.8)), \\
((0.5 / 1),(0.9 / 1))\end{array}\right)$ & $\left(\begin{array}{c}((0.6 / 1),(0.7 / 1)), \\
((0.6 / 0.4,0.8 / 0.2,0.9 / 0.4),(0.7 / 0.7,0.9 / 0.3))\end{array}\right)$ \\
$A_{2}$ & $\left(\begin{array}{c}((0.8 / 1),(0.4 / 0.6,0.5 / 0.2,0.7 / 0.2)), \\
((0.2 / 0.6,0.5 / 0.4),(0.4 / 0.3,0.5 / 0.7))\end{array}\right)$ \\
$A_{3}$ & $\left(\begin{array}{c}((0.8 / 1),(0.5 / 1)), \\
((0.7 / 1.0),(0.1 / 0.5,0.3 / 0.3,0.4 / 0.2))\end{array}\right)$ \\
$A_{4}$ & $\left(\begin{array}{c}((0.3 / 1),(0.7 / 0.7,0.8 / 0.3)), \\
((0.7 / 1),(0.6 / 1))\end{array}\right)$ & $\left(\begin{array}{c}((0.3 / 0.2,0.6 / 0.8),(0.8 / 1)), \\
((0.7 / 1),(0.3 / 1))\end{array}\right)$ \\
\hline
\end{tabular}

TABle 9: Updated expert information.

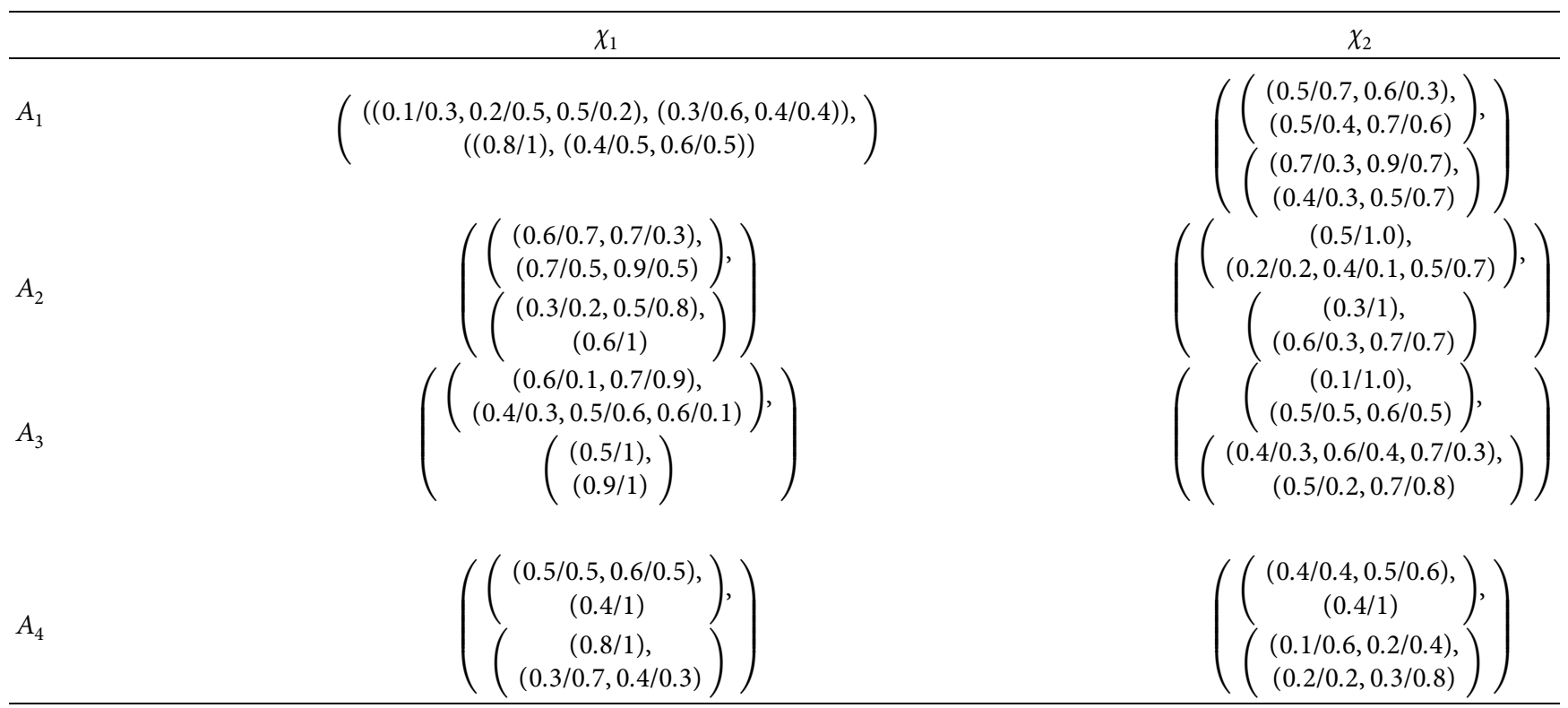


TABLE 10: Updated expert information.

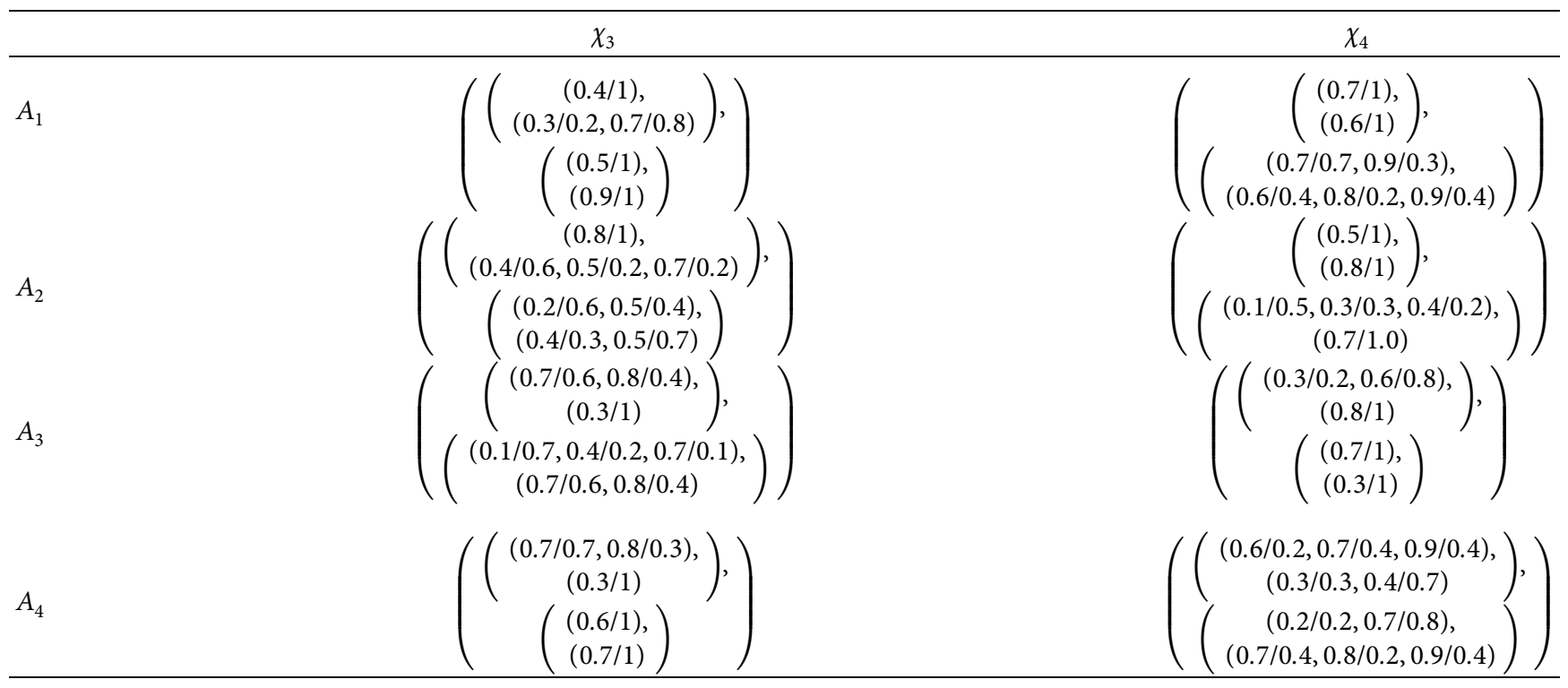

TABLE 11: Updated aggregated information using $q$-ROPHFREWA.

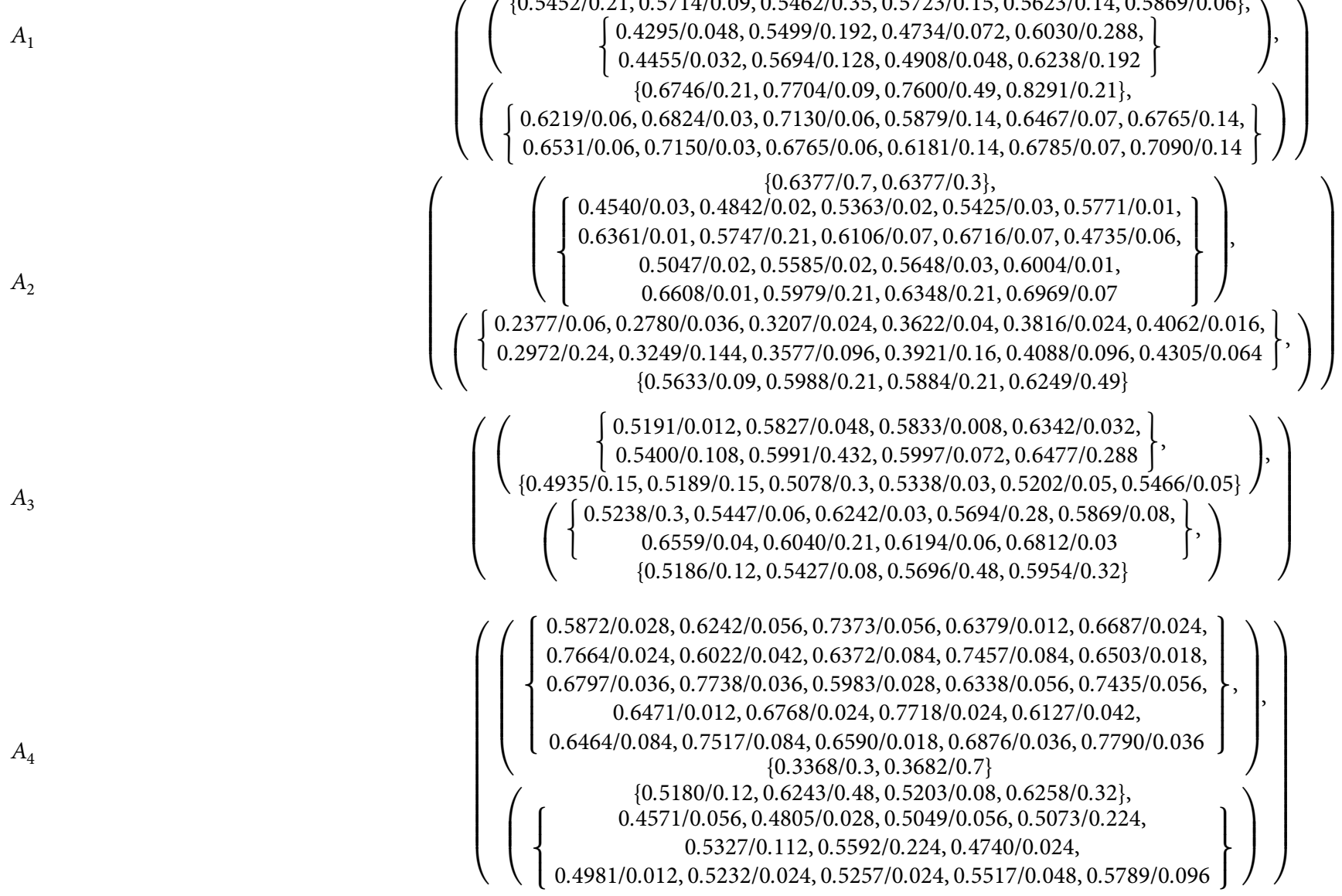


TABLE 12: Updated aggregated information using $q$-ROPHFREWG.

$A_{1}$

$A_{2}$

$A_{3}$

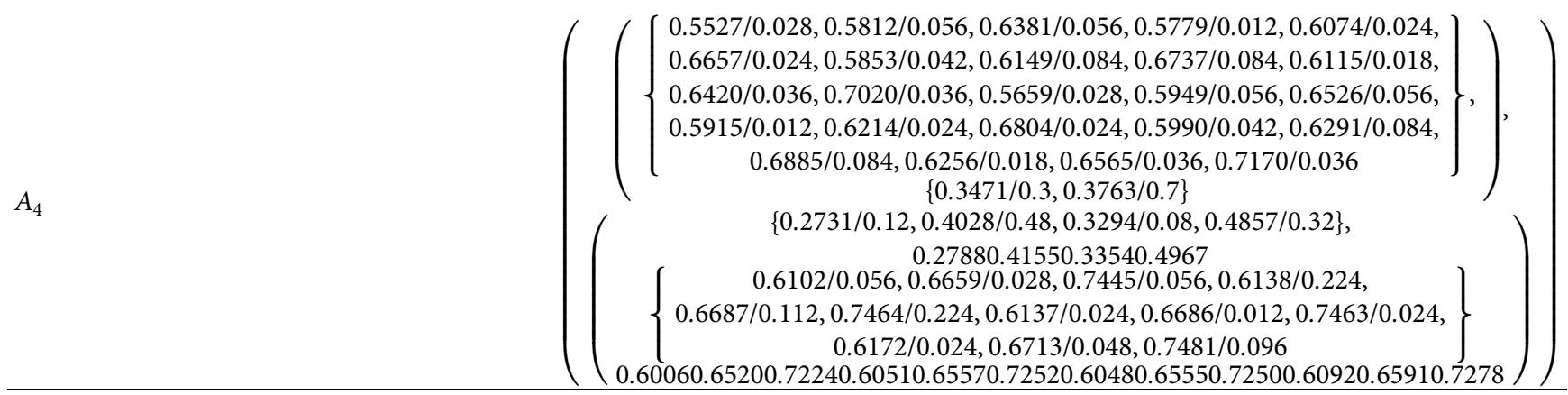

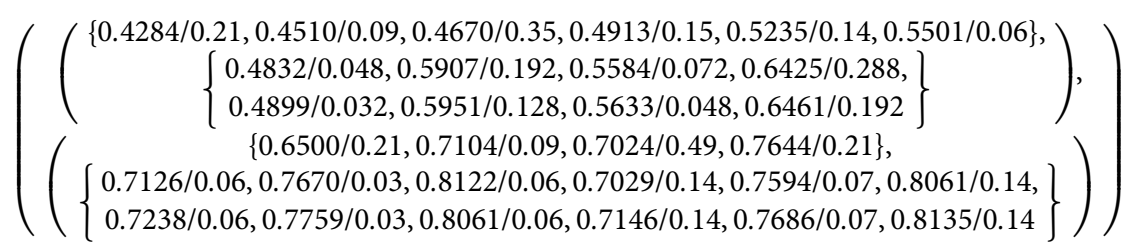

$\left(\begin{array}{c}\left\{\left(\begin{array}{c}\{0.5923 / 0.7,0.5923 / 0.3\} \\ 0.6196 / 0.03,0.6338 / 0.02,0.6814 / 0.02,0.6317 / 0.03,0.6453 / 0.01, \\ 0.6910 / 0.01,0.6444 / 0.21,0.6574 / 0.07,0.701 / 0.07,0.6741 / 0.06, \\ 0.6857 / 0.02,0.7251 / 0.02,0.6840 / 0.03,0.6952 / 0.01 \\ 0.7332 / 0.01,0.6944 / 0.21,0.7051 / 0.21,0.7418 / 0.07\end{array}\right\}\right) \\ \left(\left\{\begin{array}{c}0.1899 / 0.06,0.2668 / 0.036,0.2920 / 0.024,0.2489 / 0.04, \\ 0.3487 / 0.024,0.3811 / 0.016,0.2034 / 0.24,0.2856 / 0.144, \\ 0.3124 / 0.096,0.2664 / 0.16,0.3729 / 0.096,0.4073 / 0.064 \\ 0.5980 / 0.09,0.6134 / 0.21,0.6292 / 0.21,0.6429 / 0.49\}\end{array}\right\},\right)\end{array}\right)$ $\{0.5980 / 0.09,0.6134 / 0.21,0.6292 / 0.21,0.6429 / 0.49\}$

TABLE 13: Score values.

\begin{tabular}{lcccc}
\hline Operators & $\mathbf{G}_{\odot}\left(A_{1}\right)$ & $\mathbf{G}_{\odot}\left(A_{2}\right)$ & $\mathbf{G}_{\odot}\left(A_{3}\right)$ & $\mathbf{G}_{\Im}\left(A_{4}\right)$ \\
\hline$q$-ROPHFREWA (test) & 0.5392 & 0.5392 & 0.4852 & 0.4898 \\
-ROPHFREWG (test) & 0.5291 & 0.5299 & 0.4633 & 0.4739 \\
\hline
\end{tabular}

TABLE 14: Ranking of the alternatives.

\begin{tabular}{lll}
\hline Operators & Score & Best alternative \\
\hline$q$-ROPHFREWA (test) & $\mathbf{G}_{\odot}\left(A_{2}\right)>\mathbf{G}_{\odot}\left(A_{1}\right)>\mathbf{G}_{\odot}\left(A_{4}\right)>\mathbf{G}_{\odot}\left(A_{3}\right)$ & $A_{2}$ \\
$q$-ROPHFREWG (test) & $\mathbf{G}_{\odot}\left(A_{2}\right)>\mathbf{G}_{\odot}\left(A_{1}\right)>\mathbf{G}_{\odot}\left(A_{4}\right)>\mathbf{G}_{\odot}\left(A_{3}\right)$ & $A_{2}$ \\
\hline
\end{tabular}

where $\bar{\aleph}\left(b_{t j}\right)=\left\{\stackrel{\wedge}{g}, \beta_{h_{\bar{\aleph}(b)}}(\stackrel{\wedge}{g}) / ð_{h_{\bar{\aleph}(b)}}, \psi_{h_{\bar{\aleph}(b)}}(\stackrel{\wedge}{g}) / \partial_{h_{\bar{\aleph}(b)}} \mid \stackrel{\wedge}{g} \in \Omega\right\}$ and $\quad \underline{\aleph}(b)=\left\{\stackrel{\wedge}{g}, \beta_{h_{\underline{\aleph}(b)}}(\stackrel{\wedge}{g}) / \partial_{h_{\underline{N}(b)}}, \psi_{h_{\underline{\aleph}(b)}}(\stackrel{\wedge}{g}) / \partial_{h_{\underline{\aleph}(b)}} \mid \stackrel{\wedge}{g} \in \Omega\right\}$ such that $0 \leq\left(\max \left(\beta_{h_{\overline{\mathrm{N}}(b)}}(\stackrel{\wedge}{g})\right)\right)^{q}+\left(\min \left(\psi_{h_{\overline{\mathrm{N}}(b)}}(\stackrel{\wedge}{g})\right)\right)^{q} \leq 1$ and $0 \leq\left(\min \left(\beta_{h_{\underline{\underline{N}}(b)}}(\stackrel{\wedge}{g})\right)^{q}+\left(\max \left(\psi_{h_{\underline{\underline{N}}(b)}}(\stackrel{\wedge}{g})\right)\right)^{q} \leq 1\right.$ are the $q-$
ROHF rough values. Also, $\left.\partial_{h_{t}} \in 0,1\right], \oplus_{t=1}^{n} \partial_{h_{t}}=1$, and $\left.\partial_{h_{t}} \in 0,1\right], \oplus_{t=1}^{n} \partial_{h_{t}}=1$.

First, we collect information from DMs in the form of $q$ ROPHFRNs.

Secondly, normalize the data defined by DMs since the decision matrix may have some benefit and cost criteria all 


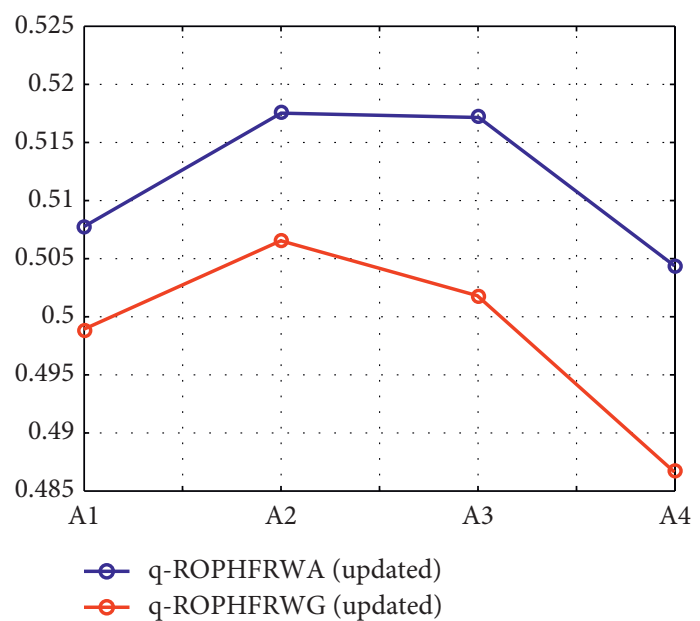

Figure 5: Alternatives using test EWA and EWG operators.

together, as shown in Equation (86), where $\hat{J}$ represents the number of experts.

Evaluate the normalized experts' matrices $(N)^{\hat{J}}$ as

$(N)^{\hat{J}}= \begin{cases}\aleph\left(b_{t j}\right)=\left(\underline{\aleph}\left(b_{t j}\right), \bar{\aleph}\left(b_{t j}\right)\right) & \text { if for benefit, } \\ \left(\aleph\left(b_{t j}\right)\right)^{c}=\left(\left(\underline{\aleph}\left(b_{t j}\right)\right)^{c},\left(\bar{\aleph}\left(b_{t j}\right)\right)^{c}\right) & \text { if for cost. }\end{cases}$

$$
\begin{aligned}
\Upsilon^{+} & =\left(\tau_{1}^{+}, \tau_{2}^{+}, \tau_{3}^{+}, \ldots \tau_{n}^{+}\right) \\
& =\left(\max _{t} \operatorname{score}\left(\tau_{t 1}\right), \max _{t} \operatorname{score}\left(\tau_{t 2}\right), \max _{t} \operatorname{score}\left(\tau_{t 3}\right), \ldots, \max _{t} \operatorname{score}\left(\tau_{t n}\right)\right) .
\end{aligned}
$$

Likewise, the NIS is calculated by the formula as follows:

$$
\begin{aligned}
\Upsilon^{-} & =\left(\tau_{1}^{-}, \tau_{2}^{-}, \tau_{3}^{-}, \ldots, \tau_{n}^{-}\right) \\
& =\left(\min _{t} \operatorname{score}\left(\tau_{t 1}\right), \min _{t} \operatorname{score}\left(\tau_{t 2}\right), \min _{t} \operatorname{score}\left(\tau_{t 3}\right), \ldots, \min _{t} \operatorname{score}\left(\tau_{t n}\right)\right) .
\end{aligned}
$$

Afterward, find the geometric distance between all the alternatives and PI $I^{+}$as follows:

$$
d\left(\alpha_{t j}, \Upsilon^{+}\right)=\frac{1}{8}\left(\begin{array}{c}
\left(\frac{1}{\# h} \sum_{s=1}^{\# h}\left|\left(\bar{\Phi}_{t j(s)} \times \bar{\partial}_{\epsilon_{t(s)}^{+}}\right)^{2}-\left(\bar{\Phi}_{t(s)}^{+} \times \bar{\partial}_{t(s)}^{+}\right)^{2}\right|+\left|\left(\bar{\Phi}_{t j(s)} \times \bar{\partial}_{\epsilon_{t(s)}^{+}}^{+}\right)^{2}-\left(\bar{\Phi}_{t(s)}^{+} \times \bar{\partial}_{\epsilon t(s)}^{+}\right)^{2}\right|\right) \\
\left.+\left(\left(\frac{1}{\# g} \sum_{s=1}^{\# g}\left|\left(\underline{v}_{t j(s)} \times \partial_{\epsilon_{t j(s)}}\right)^{2}-\left(\underline{v}_{t(s)}^{+} \times \partial_{\epsilon_{t}(s)}^{+}\right)^{2}\right|+\left|\left(\bar{\nu}_{h} \times \bar{\partial}_{\epsilon_{t}(s)}^{+}\right)^{2}-\left(\bar{\nu}_{h}^{+} \times \bar{\partial}_{\epsilon_{t}(s)}^{+}\right)^{2}\right|\right)\right)\right)
\end{array}\right),
$$




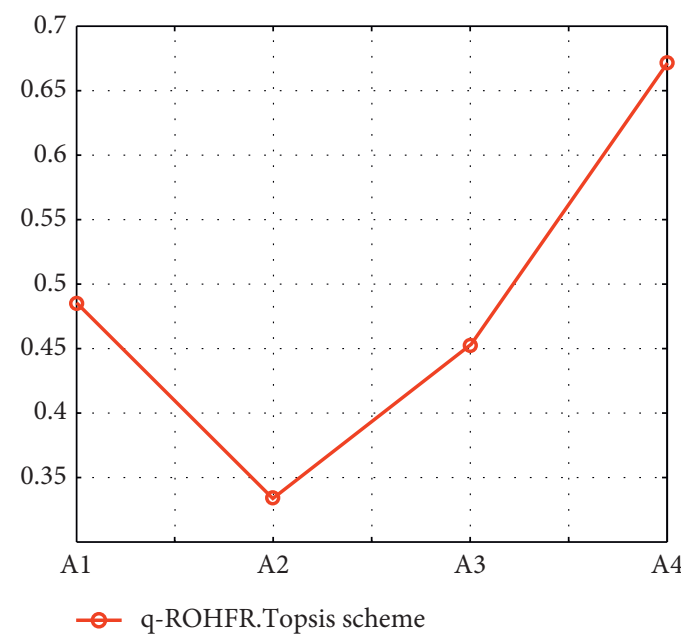

Figure 6: Alternatives using the TOPSIS technique.

TABLE 15: Score values of expert information.

\begin{tabular}{lcccc}
\hline & $\chi_{1}$ & $\chi_{2}$ & $\chi_{3}$ & $\chi_{4}$ \\
\hline$A_{1}$ & 0.6142 & 0.4600 & 0.4225 & 0.4433 \\
$A_{2}$ & 0.3825 & 0.4196 & 0.6412 & 0.7317 \\
$A_{3}$ & 0.5538 & 0.4213 & 0.5567 & 0.4675 \\
$A_{4}$ & 0.3725 & 0.4400 & 0.5087 & 0.5254 \\
\hline
\end{tabular}

where $t=1,2,3, \ldots, n$ and $j=1,2,3, \ldots, m . s$ is a positive number which represents the number of elements contained in $q$-ROPHFRS. Analogously, the geometric distance between all the alternatives and NI $\Upsilon^{-}$is as follows:

$$
d\left(\alpha_{t j}, \Upsilon^{-}\right)=\frac{1}{8}\left(\begin{array}{c}
\left(\frac{1}{\# h} \sum_{s=1}^{\# h}\left|\left(\bar{\Phi}_{t j(s)} \times \underline{\partial}_{\epsilon_{t j(s)}}\right)^{2}-\left(\overline{\bar{\omega}}_{t(s)}^{-} \times \bar{\partial}_{t(s)}^{-}\right)^{2}\right|+\left|\left(\overline{\bar{\omega}}_{t j(s)} \times{\overline{\partial_{\epsilon}}}_{\epsilon_{t(s)}}^{+}\right)^{2}-\left(\overline{\bar{\omega}}_{t(s)}^{-} \times \bar{\partial}_{\epsilon t(s)}^{-}\right)^{2}\right|\right) \\
+\left(\left(\frac{1}{\# g} \sum_{s=1}^{\# g}\left|\left(\underline{v}_{t j(s)} \times \partial_{\epsilon_{t j(s)}}\right)^{2}-\left(\underline{v}_{t(s)}^{-} \times \partial_{\epsilon_{t}(s)}^{-}\right)^{2}\right|+\left|\left(\bar{v}_{h_{t j}} \times \bar{\partial}_{\epsilon_{t j(s)}}\right)^{2}-\left(\bar{\nu}_{h_{t}}^{-} \times \bar{\partial}_{\epsilon_{t}(s)}^{-}\right)^{2}\right|\right)\right.
\end{array}\right),
$$

where $t=1,2,3, \ldots, n$ and $j=1,2,3, \ldots, m$.

The relative closeness indices for all DMs of the alternatives are calculated as follows:

$$
\operatorname{RC}\left(\alpha_{t j}\right)=\frac{d\left(\alpha_{t j}, \Upsilon^{+}\right)}{d\left(\alpha_{t j}, \Upsilon^{-}\right)+d\left(\alpha_{t j}, \Upsilon^{+}\right)}
$$

The ranking orders of alternatives can be determined, and the most desirable alternative having minimum distance is chosen.

\section{Implementation of the Methodology}

A numerical example relevant to "drug selection for the treatment of COVID-19 disease" is given below to validate the usefulness of our approach.
The DM information in the form of $q$-ROPHFRNs is given in Tables 1 and 2.

PIS and NIS are computed in Table 13.

Compute the distance measure of the PIS and NIS.

\begin{tabular}{|l|l|l|l|}
\hline 0.3799 & 0.2158 & 0.4174 & 0.4971 \\
\hline 0.4038 & 0.4301 & 0.5054 & 0.2434 \\
\hline
\end{tabular}

The relative closeness indices for all DM of the alternatives are calculated.

\begin{tabular}{|l|l|l|l|}
\hline 0.4848 & 0.3341 & 0.4523 & 0.6713 \\
\hline
\end{tabular}

From ranking of the alternative, it could be seen that $A_{2}$ has the minimum distance (Figure 6). Hence, $A_{2}$ is the best alternative (Tables 15-17). 
TABLE 16: Ideal solutions.

\begin{tabular}{|c|c|c|}
\hline Criteria & $\Upsilon^{+}$ & $\Upsilon^{-}$ \\
\hline$\chi_{1}$ & $\left(\begin{array}{c}\left(\begin{array}{c}(0.1 / 0.3,0.2 / 0.5,0.5 / 0.2) \\
(0.3 / 0.6,0.4 / 0.4)\end{array}\right) \\
\left(\begin{array}{c}(0.8 / 1) \\
(0.4 / 0.5,0.6 / 0.5)\end{array}\right)\end{array}\right.$ & $\left(\begin{array}{c}\left(\begin{array}{c}(0.4 / 1), \\
(0.5 / 0.5,0.6 / 0.5)\end{array}\right) \\
\left(\begin{array}{c}(0.3 / 0.7,0.4 / 0.3), \\
(0.8 / 1)\end{array}\right)\end{array}\right)$ \\
\hline$\chi_{2}$ & $\left(\begin{array}{c}\left(\begin{array}{c}(0.5 / 0.4,0.7 / 0.6) \\
(0.5 / 0.7,0.6 / 0.3)\end{array}\right) \\
\left(\begin{array}{c}(0.4 / 0.3,0.5 / 0.7), \\
(0.7 / 0.3,0.9 / 0.7)\end{array}\right)\end{array}\right)$ & $\begin{array}{c}\left(\begin{array}{c}(0.2 / 0.2,0.4 / 0.1,0.5 / 0.7) \\
(0.5 / 1.0)\end{array}\right) \\
\left(\begin{array}{c}(0.6 / 0.3,0.7 / 0.7), \\
(0.3 / 1)\end{array}\right)\end{array}$ \\
\hline$\chi_{3}$ & $\left(\begin{array}{c}(0.8 / 1), \\
(0.4 / 0.6,0.5 / 0.2,0.7 / 0.2)\end{array}\right)$ & $\left(\begin{array}{c}(0.4 / 1), \\
(0.3 / 0.2,0.7 / 0.8)\end{array}\right)$, \\
\hline$\chi_{4}$ & $\left(\begin{array}{c}\left(\begin{array}{c}(0.8 / 1) \\
(0.5 / 1)\end{array}\right) \\
(0.7 / 1.0) \\
(0.1 / 0.5,0.3 / 0.3,0.4 / 0.2)\end{array}\right.$ & $\begin{array}{c}\left(\begin{array}{c}(0.6 / 1) \\
(0.7 / 1)\end{array}\right) \\
\left(\begin{array}{c}(0.6 / 0.4,0.8 / 0.2,0.9 / 0.4), \\
(0.7 / 0.7,0.9 / 0.3)\end{array}\right)\end{array}$ \\
\hline
\end{tabular}

TABLE 17: Ranking of the alternatives.

\begin{tabular}{lc}
\hline Score & Best alternative \\
\hline $\mathbf{G}_{\odot}\left(A_{2}\right)>\mathbf{G}_{\odot}\left(A_{3}\right)>\mathbf{G}_{\odot}\left(A_{1}\right)>\mathbf{G}_{\odot}\left(A_{4}\right)$ & $A_{2}$ \\
\hline
\end{tabular}

\section{Conclusion}

The main objective of this research is to present a unique and new approach for evaluating the medicine selection problem for COVID-19 infection utilizing the novel concepts of $q$ ROPHFRS under the $q$-ROFS, HFS, and RS environment. In comparison to conventional fuzzy models, a $q$-ROPHFR model effectively describes real-world problems with ambiguity, vagueness, and incompleteness. Furthermore, we introduced the concept of $q$-ROPHFREWA and $q$-ROPHFREWG aggregation operators that are effective and flexible to MCGDM problems with uncertainty. Furthermore, the efficiency and applicability of the described notion have been presented by evaluating medications for the patients infected by the COVID-19 virus. In the final ranking and optimal decision-making of treatments for patients of COVID-19 virus, the proposed techniques are compared with the $q$-ROHFR-TOPSIS method, and the superiority of the acquired results is validated. The ranking of the alternatives is depicted graphically. According to the final ranking of the established methodology, remdesivir is the most effective medicine for treating patients infected with COVID-19.

It is observed that some essential topics remain in terms of potential future works that are good enough to justify. In the future, this work will be extended to the (1) q-ROPHFRE ordered weighted averaging operator (WAO); (2) $q$-ROPHFRE hybrid AOPs; (3) q-ROPHFRE ordered weighted geometric operator (WGO); and (4) $q$-ROPHFRE hybrid WGO.

12.1. Limitation. A number of included studies were limited in terms of data availability and methodological quality. Therefore, the reported findings should be interpreted cautiously within that context. Furthermore, our study was limited to the articles published in English. Considering the epicenter of COVID-19, Chinese literature should be included in future systematic reviews. We will continue to monitor the literature, and this method will be updated when new evidence emerges.

\section{Data Availability}

The data used in the manuscript are hypothetical and can be used by anyone by just citing this article.

\section{Conflicts of Interest}

The authors declare no conflicts of interest about the publication of the research article.

\section{Acknowledgments}

This study was supported by the Researchers Supporting Project number (RSP-2021/244), King Saud University, Riyadh, Saudi Arabia.

\section{References}

[1] M. A. Martinez, "Comaleph with therapeutic potential against novel respiratory 2019 coronavirus," Antimicrobial Agents and Chemotherapy, vol. 64, no. 5, p. e00399, 2020.

[2] Worldometer, "COVID-19 Coronavirus Pandemic," 2020, http://www.worldometers.info/coronavirus/\#countries\%3C.

[3] T.-M. Chen, J. Rui, Q.-P. Wang, Z.-Y. Zhao, J.-A. Cui, and L. Yin, "A mathematical model for simulating the phase-based transmissibility of a novel coronavirus," Infectious Diseases of Poverty, vol. 9, no. 1, p. 24, 2020.

[4] R. Lu, X. Zhao, J. Li et al., "Genomic characterisation and epidemiology of 2019 novel coronavirus: implications for virus origins and receptor binding," The Lancet, vol. 395, no. 10224, pp. 565-574, 2020. 
[5] L. T. Phan, T. V. Nguyen, Q. C. Luong et al., "Importation and human-to-human transmission of a novel coronavirus in Vietnam," New England Journal of Medicine, vol. 382, no. 9, pp. 872-874, 2020.

[6] Z. Xu, L. Shi, Y. Wang et al., "Pathological findings of COVID19 associated with acute respiratory distress syndrome," The Lancet respiratory medicine, vol. 8, no. 4, pp. 420-422, 2020.

[7] Y. Huang, S. Wang, Y. Liu et al., "A preliminary study on the ultrasonic manifestations of peripulmonary lesions of noncritical novel coronavirus pneumonia (COVID-19)," 2020.

[8] A. R. Mishra, P. Rani, R. Krishankumar, K. S. Ravichandran, and S. Kar, "An extended fuzzy decision-making framework using hesitant fuzzy sets for the drug selection to treat the mild symptoms of Coronavirus disease 2019 (COVID-19)," Applied Soft Computing, vol. 103, Article ID 107155, 2021.

[9] A. M. Zaki, S. Van Boheemen, T. M. Bestebroer, A. D. M. E. Osterhaus, and R. A. M. Fouchier, "Isolation of a novel coronavirus from a man with pneumonia in Saudi Arabia," New England Journal of Medicine, vol. 367, no. 19, pp. 1814-1820, 2012.

[10] P. Zhou, X.-L. Yang, X.-G. Wang et al., "A pneumonia outbreak associated with a new coronavirus of probable bat origin," Nature, vol. 579, no. 7798, pp. 270-273, 2020.

[11] N. Zhu, D. Zhang, W. Wang et al., "A novel coronavirus from patients with pneumonia in China, 2019," New England Journal of Medicine, vol. 382, no. 8, pp. 727-733, 2020.

[12] N. Chen, M. Zhou, X. Dong et al., "Epidemiological and clinical characteristics of 99 cases of 2019 novel coronavirus pneumonia in Wuhan, China: a descriptive study," The Lancet, vol. 395, no. 10223, pp. 507-513, 2020.

[13] J. F.-W. Chan, S. Yuan, K.-H. Kok et al., "A familial cluster of pneumonia associated with the 2019 novel coronavirus indicating person-to-person transmission: a study of a family cluster," The Lancet, vol. 395, no. 10223, pp. 514-523, 2020.

[14] WHO, Novel Coronavirus (2019-nCoV) Situation Reports, http://www.who.int/emergencies/diseases/novelcoronavirus2019/situation-reports/, World Health Organization, Geneva, Switzerland, 2020, http://www.who.int/emergencies/diseases/ novelcoronavirus-2019/situation-reports/.

[15] L. A. Zadeh, "Fuzzy sets," Information and Control, vol. 8, no. 3, pp. 338-353, 1965.

[16] K. T. Atanassov, "Intuitionistic fuzzy sets," in Intuitionistic Fuzzy Sets, pp. 1-137, Physica, Heidelberg, Germany, 1999.

[17] R. R. Yager, "Pythagorean membership grades in multicriteria decision making," IEEE Transactions on Fuzzy Systems, vol. 22, no. 4, pp. 958-965, 2013.

[18] C. Huang, M. Lin, and Z. Xu, "Pythagorean fuzzy MULTIMOORA method based on distance measure and score function: its application in multicriteria decision making process," Knowledge and Information Systems, vol. 62, no. 11, pp. 4373-4406, 2020.

[19] X. Zhang and Z. Xu, "Extension of TOPSIS to multiple criteria decision making with Pythagorean fuzzy sets," International Journal of Intelligent Systems, vol. 29, no. 12, pp. 1061-1078, 2014.

[20] V. Torra, "Hesitant fuzzy sets," International Journal of Intelligent Systems, vol. 25, no. 6, pp. 529-539, 2010.

[21] J. Liu and M. Sun, "Generalized power average operator of hesitant fuzzy numbers and its application in multiple attribute decision making," Journal of Computational Information Systems, vol. 9, no. 8, pp. 3051-3058, 2013.

[22] M. Xia and Z. Xu, "Hesitant fuzzy information aggregation in decision making," International Journal of Approximate Reasoning, vol. 52, no. 3, pp. 395-407, 2011.
[23] Z. Xu, "Intuitionistic fuzzy aggregation operators," IEEE Transactions on Fuzzy Systems, vol. 15, no. 6, pp. 1179-1187, 2007.

[24] Z. Xu and R. R. Yager, "Some geometric aggregation operators based on intuitionistic fuzzy sets," International Journal of General Systems, vol. 35, no. 4, pp. 417-433, 2006.

[25] H. Liao and Z. Xu, "Extended hesitant fuzzy hybrid weighted aggregation operators and their application in decision making," Soft Computing, vol. 19, no. 9, pp. 2551-2564, 2015.

[26] M. S. A. Khan, S. Abdullah, A. Ali, N. Siddiqui, and F. Amin, "Pythagorean hesitant fuzzy sets and their application to group decision making with incomplete weight information," Journal of Intelligent and Fuzzy Systems, vol. 33, no. 6, pp. 3971-3985, 2017.

[27] Z. Xu and W. Zhou, "Consensus building with a group of decision makers under the hesitant probabilistic fuzzy environment," Fuzzy Optimization and Decision Making, vol. 16, no. 4, pp. 481-503, 2017.

[28] Z. Hao, Z. Xu, H. Zhao, and Z. Su, "Probabilistic dual hesitant fuzzy set and its application in risk evaluation," KnowledgeBased Systems, vol. 127, pp. 16-28, 2017.

[29] J. Li and Z.-x. Wang, "Multi-attribute decision making based on prioritized operators under probabilistic hesitant fuzzy environments," Soft Computing, vol. 23, no. 11, pp. 3853-3868, 2019.

[30] W. Zhou and Z. Xu, "Group consistency and group decision making under uncertain probabilistic hesitant fuzzy preference environment," Information Sciences, vol. 414, pp. 276288, 2017.

[31] R. R. Yager, "Generalized orthopair fuzzy sets," IEEE Transactions on Fuzzy Systems, vol. 25, no. 5, pp. 1222-1230, 2016.

[32] R. R. Yager and N. Alajlan, "Approximate reasoning with generalized orthopair fuzzy sets," Information Fusion, vol. 38, pp. 65-73, 2017.

[33] D. Liu, D. Peng, and Z. Liu, "The distance measures between q-rung orthopair hesitant fuzzy sets and their application in multiple criteria decision making," International Journal of Intelligent Systems, vol. 34, no. 9, pp. 2104-2121, 2019.

[34] J. Wang, P. Wang, G. Wei, C. Wei, and J. Wu, "Some power Heronian mean operators in multiple attribute decisionmaking based on q-rung orthopair hesitant fuzzy environment," Journal of Experimental \& Theoretical Artificial Intelligence, vol. 32, no. 6, pp. 909-937, 2020.

[35] J. Wang, G. Wei, C. Wei, and Y. Wei, "Dual hesitant q-Rung Orthopair fuzzy Muirhead mean operators in multiple attribute decision making," Ieee Access, vol. 7, pp. 67139-67166, 2019.

[36] Z. Hussain and M.-S. Yang, "Entropy for hesitant fuzzy sets based on Hausdorff metric with construction of hesitant fuzzy TOPSIS," International Journal of Fuzzy Systems, vol. 20, no. 8, pp. 2517-2533, 2018.

[37] C. L. Hwang and K. S. Yoon, Multiple Attribute Decision Methods and Applications, pp. 673-674, Springer, Berlin, German, 1981.

[38] F. E. Boran, S. Genç, M. Kurt, and D. Akay, "A multi-criteria intuitionistic fuzzy group decision making for supplier selection with TOPSIS method," Expert Systems with Applications, vol. 36, no. 8, pp. 11363-11368, 2009.

[39] T.-Y. Chen and C.-Y. Tsao, "The interval-valued fuzzy TOPSIS method and experimental analysis," Fuzzy Sets and Systems, vol. 159, no. 11, pp. 1410-1428, 2008.

[40] Y. Wang, Z. Shan, and L. Huang, "The extension of TOPSIS method for multi-attribute decision-making with q-Rung 
orthopair hesitant fuzzy sets," IEEE Access, vol. 8, pp. 165151-165167, 2020.

[41] D. F. Li, "TOPSIS-based nonlinear-programming methodology for multiattribute decision making with interval-valued intuitionistic fuzzy sets," IEEE Transactions on Fuzzy Systems, vol. 18, no. 2, pp. 299-311, 2010.

[42] J. H. Park, I. Y. Park, Y. C. Kwun, and X. Tan, "Extension of the TOPSIS method for decision making problems under interval-valued intuitionistic fuzzy environment," Applied Mathematical Modelling, vol. 35, no. 5, pp. 2544-2556, 2011.

[43] A. Khan, S. Ashraf, S. Abdullah, M. Qiyas, J. Luo, and S. Khan, "Pythagorean fuzzy Dombi aggregation operators and their application in decision support system," Symmetry, vol. 11, no. 3, p. 383, 2019.

[44] O. Barukab, S. Abdullah, S. Ashraf, M. Arif, and S. A. Khan, "A new approach to fuzzy TOPSIS method based on entropy measure under spherical fuzzy information," Entropy, vol. 21, no. 12, p. 1231, 2019.

[45] I. Beg and T. Rashid, "TOPSIS for hesitant fuzzy linguistic term sets," International Journal of Intelligent Systems, vol. 28, no. 12, pp. 1162-1171, 2013.

[46] A. Biswas and B. Sarkar, "Pythagorean fuzzy TOPSIS for multicriteria group decision-making with unknown weight information through entropy measure," International Journal of Intelligent Systems, vol. 34, no. 6, pp. 1108-1128, 2019.

[47] Z. Xu and X. Zhang, "Hesitant fuzzy multi-attribute decision making based on TOPSIS with incomplete weight information," Knowledge-Based Systems, vol. 52, pp. 53-64, 2013.

[48] Z. a. Pawlak, "Rough sets," International Journal of Computer \& Information Sciences, vol. 11, no. 5, pp. 341-356, 1982.

[49] C. H. Su, K. T. K. Chen, and K. K. Fan, "Rough set theory based fuzzy TOPSIS on serious game design evaluation framework," Mathematical Problems in Engineering, vol. 2013, Article ID 407395, 13 pages, 2013.

[50] C. Khan, S. Anwar, S. Bashir, A. Rauf, and A. Amin, "Site selection for food distribution using rough set approach and TOPSIS method," Journal of Intelligent and Fuzzy Systems, vol. 29, no. 6, pp. 2413-2419, 2015.

[51] J. Lu and Z. Zhao, "Improved TOPSIS based on rough set theory for selection of suppliers," in Proceedings of the 2008 4th International Conference on Wireless Communications, Networking and Mobile Computing, pp. 1-4, IEEE, Dalian, China, October 2008.

[52] B. Yu, M. Cai, and Q. Li, “A $\lambda$-rough set model and its applications with TOPSIS method to decision making b-rough set model and its applications with TOPSIS method to decision making," Knowledge-Based Systems, vol. 165, pp. 420431, 2019.

[53] D. Dubois and H. Prade, "Rough fuzzy sets and fuzzy rough sets," International Journal of General Systems, vol. 17, no. 2-3, pp. 191-209, 1990.

[54] C. Cornelis, M. De Cock, and E. E. Kerre, "Intuitionistic fuzzy rough sets: at the crossroads of imperfect knowledge," Expert Systems, vol. 20, no. 5, pp. 260-270, 2003.

[55] L. Zhou and W. Z. Wu, "On generalized intuitionistic fuzzy rough approximation operators," Information Sciences, vol. 178, no. 11, pp. 2448-2465, 2008.

[56] J. Zhan, H. Masood Malik, and M. Akram, "Novel decisionmaking algorithms based on intuitionistic fuzzy rough environment," International Journal of Machine Learning and Cybernetics, vol. 10, no. 6, pp. 1459-1485, 2019.

[57] S. M. Yun and S. J. Lee, "Intuitionistic fuzzy rough approximation operators," The International Journal of Fuzzy Logic and Intelligent Systems, vol. 15, no. 3, pp. 208-215, 2015.
[58] C. Zhang, "Classification rule mining algorithm combining intuitionistic fuzzy rough sets and genetic algorithm," International Journal of Fuzzy Systems, vol. 22, no. 5, pp. 1694-1715, 2020.

[59] R. Chinram, A. Hussain, T. Mahmood, and M. I. Ali, "EDAS method for multi-criteria group decision making based on intuitionistic fuzzy rough aggregation operators," IEEE Access, vol. 9, pp. 10199-10216, 2021.

[60] R. Verma and B. D. Sharma, "New operations over hesitant fuzzy sets," Fuzzy Information and Engineering, vol. 5, no. 2, pp. 129-146, 2013.

[61] M. A. Shereen, S. Khan, A. Kazmi, N. Bashir, and R. Siddique, "COVID-19 infection: e," Journal of Advanced Research, vol. 24, pp. 91-98, 2020.

[62] G. Büyüközkan and M. Güler, "Analysis of companies' digital maturity by hesitant fuzzy linguistic MCDM methods," Journal of Intelligent and Fuzzy Systems, vol. 38, no. 1, pp. 1119-1132, 2020.

[63] J. Dahooie, E. Zavadskas, M. Abolhasani, A. Vanaki, and Z. Turskis, "A novel approach for evaluation of projects using an interval-valued fuzzy additive ratio assessment (ARAS) method: a case study of oil and gas well drilling projects," Symmetry, vol. 10, no. 2, p. 45, 2018.

[64] C. Ghenai, M. Albawab, and M. Bettayeb, "Sustainability indicators for renewable energy systems using multi-criteria decision-making model and extended SWARA/ARAS hybrid method," Renewable Energy, vol. 146, pp. 580-597, 2020.

[65] H. Liao, Z. Wen, and L. Liu, "Integrating BWM and ARAS under hesitant linguistic environment for digital supply chain finance supplier section," Technological and Economic Development of Economy, vol. 25, no. 6, pp. 1188-1212, 2019.

[66] G. Li and E. De Clercq, "Therapeutic options for the 2019 novel coronavirus (2019-nCoV)," Nature Reviews Drug Discovery, vol. 19, no. 3, pp. 149-150, 2020.

[67] S. Mulangu, L. E. Dodd, R. T. Davey Jr. et al., "A randomized, controlled trial of Ebola virus disease therapeutics," New England Journal of Medicine, vol. 381, no. 24, pp. 2293-2303, 2019.

[68] J. S. Morse, T. Lalonde, S. Xu, and W. R. Liu, "Learning from the past: possible urgent prevention and treatment options for severe acute respiratory infections caused by 2019-nCoV," ChemBioChem, vol. 21, no. 5, pp. 730-738, 2020.

[69] M. Wang, R. Cao, L. Zhang et al., "Remdesivir and chloroquine effectively inhibit the recently emerged novel coronavirus (2019-nCoV) in vitro," Cell Research, vol. 30, no. 3, pp. 269-271, 2020.

[70] W. Alhazzani, M. H. Møller, Y. M. Arabi et al., "Surviving sepsis campaign: guidelines on the management of critically ill adults with Coronavirus disease 2019 (COVID-19)," Intensive Care Medicine, vol. 46, no. 5, pp. 1-34, 2020.

[71] C. Huang, Y. Wang, X. Li et al., "Clinical features of patients infected with 2019 novel coronavirus in Wuhan, China," The Lancet, vol. 395, no. 10223, pp. 497-506, 2020.

[72] Z. Ren, H. Liao, and Y. Liu, "Generalized Z-numbers with hesitant fuzzy linguistic information and its application to medicine selection for the patients with mild symptoms of the COVID-19," Computers \& Industrial Engineering, vol. 145, Article ID 106517, 2020.

[73] C. Wu, X. Chen, Y. Cai et al., "Risk factors associated with acute respiratory distress syndrome and death in patients with coronavirus disease 2019 pneumonia in Wuhan, China," JAMA Internal Medicine, vol. 180, no. 7, pp. 934-943, 2020.

[74] X.-W. Xu, X.-X. Wu, X.-G. Jiang et al., "Clinical findings in a group of patients infected with the 2019 novel coronavirus 
(SARS-CoV-2) outside of Wuhan, China: retrospective case series," BMJ, vol. 368, p. m606, 2020.

[75] L. Wang and H. Garg, "Algorithm for multiple attribute decision-making with interactive archimedean norm operations under pythagorean fuzzy uncertainty," International Journal of Computational Intelligence Systems, vol. 14, no. 1, pp. 503-527, 2021.

[76] P.-F. Hsu and M.-G. Hsu, "Optimizing the information outsourcing practices of primary care medical organizations using entropy and TOPSIS," Quality and Quantity, vol. 42, no. 2, pp. 181-201, 2008.

[77] G. H. Tzeng and J. J. Huang, Multiple Attribute Decision Making: Methods and Applications, CRC Press, Boca Raton, FL, USA, 2011. 